\title{
Compact D-D/D-T Neutron Generators and Their Applications
}

\author{
by
}

Tak Pui Lou

B.S. (University of Florida) 1998

M.S. (University of California, Berkeley) 2000

A dissertation (or thesis) submitted in partial satisfaction of the requirements for the degree of

Doctor of Philosophy

in

Engineering - Nuclear Engineering

in the

GRADUATE DIVISION

of the

\section{UNIVERSITY OF CALIFORNIA, BERKELEY}

\author{
Committee in charge: \\ Professor Jasmina L. Vujic, Chair \\ Professor Ka-Ngo Leung \\ Professor Per F. Peterson \\ Professor Hans-Rudolf Wenk
}

Spring 2003 
The dissertation (or thesis) of Tak Pui Lou is approved:

\begin{tabular}{ll}
\hline Chair & Date \\
\hline & Date \\
\hline & Date \\
\hline
\end{tabular}

Date

University of California, Berkeley

Spring 2003 


\section{Compact D-D/D-T Neutron Generators and Their Applications}

Copyright 2003

by

Tak Pui Lou 



\begin{abstract}
Compact D-D/D-T Neutron Generators and Their Applications

by

Tak Pui Lou

Doctor of Philosophy in Engineering - Nuclear Engineering

University of California, Berkeley

Professor Jasmina L. Vujic, Chair
\end{abstract}

Neutron generators based on the ${ }^{2} \mathrm{H}(\mathrm{d}, \mathrm{n}){ }^{3} \mathrm{He}$ and ${ }^{3} \mathrm{H}(\mathrm{d}, \mathrm{n}){ }^{4} \mathrm{He}$ fusion reactions are the most commonly available neutron sources. The applications of current commercial neutron generators are often limited by their low neutron yield and their short operational lifetime. A new generation of D-D/D-T fusion-based neutron generators has been designed at Lawrence Berkeley National Laboratory (LBNL) by using high current ion beams hitting on a self-loading target that has a large surface area to dissipate the heat load.

This thesis describes the rationale behind the new designs and their potential applications. A survey of other neutron sources is presented to show their advantages and disadvantages compared to the fusion-based neutron generator. A prototype neutron facility was built at LBNL to test these neutron generators. High current ion beams were extracted from an RF-driven ion source to produce neutrons. With an average deuteron beam current of $24 \mathrm{~mA}$ and an energy of $100 \mathrm{keV}$, a neutron yield of $>10^{9} \mathrm{n} / \mathrm{s}$ has been obtained with a D-D coaxial neutron source. 
Several potential applications were investigated by using computer simulations. The computer code used for simulations and the variance reduction techniques employed were discussed. A study was carried out to determine the neutron flux and resolution of a D-T neutron source in thermal neutron scattering applications for condensed matter experiments. An error analysis was performed to validate the scheme used to predict the resolution. With a D-T neutron yield of $10^{14} \mathrm{n} / \mathrm{s}$, the thermal neutron flux at the sample was predicted to be $7.3 \times 10^{5} \mathrm{n} / \mathrm{cm}^{2} \mathrm{~s}$. It was found that the resolution of cold neutrons was better than that of thermal neutrons when the duty factor is high. This neutron generator could be efficiently used for research and educational purposes at universities.

Additional applications studied were positron production and Boron Neutron Capture Therapy (BNCT). The neutron flux required for positron production could not be provided with a single D-T neutron generator. Therefore, a subcritical fission multiplier was designed to increase the neutron yield. The neutron flux was increased by a factor of 25. A D-D driven fission multiplier was also studied for BNCT and a gain of 17 was obtained. The fission multiplier system gain was shown to be limited by the neutron absorption in the fuel and the reduction of source brightness. A brief discussion was also given regarding the neutron generator applications for fast neutron brachytherapy and neutron interrogation systems. It was concluded that new designs of compact D-D/D-T neutron generators are feasible and that superior quality neutron beams could be produced and used for various applications.

Professor Jasmina Vujic

Dissertation Committee Chair 


\section{Table of Contents}

List of Figures.......................................................................................................................

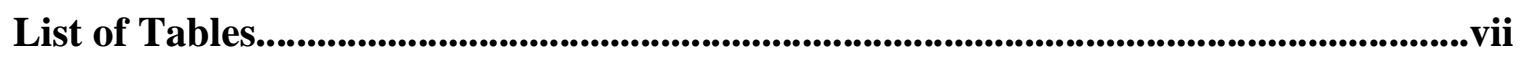

Acknowledgements................................................................................................................................viii

1 Introduction...........................................................................................................................1

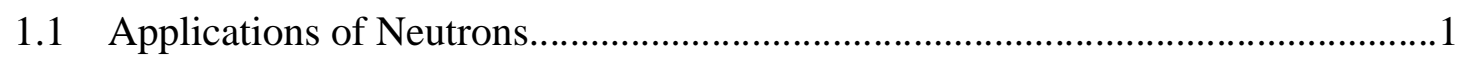

1.2 Properties of fusion-based neutron generators.......................................................

1.2.1 Monoenergetic fusion-based neutron sources..............................................

1.2.2 Fusion-based white neutron source...........................................................

1.3 Scope of thesis research...............................................................................

2 Survey of other neutron sources and their properties....................................................8

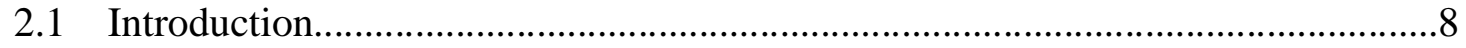

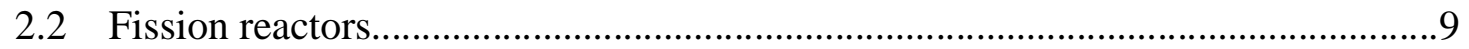

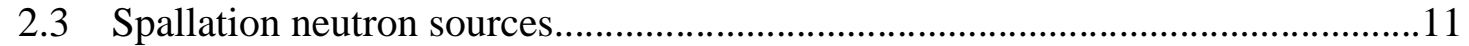

2.4 Charge particles induced neutron sources...................................................13

2.5 Radioisotope neutron sources.....................................................................13

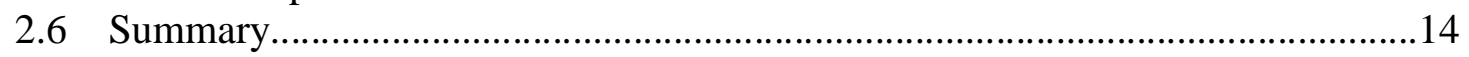

3 New Fusion-based Neutron Generator Designs.............................................................15

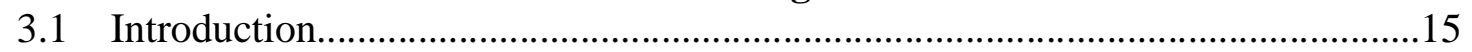

3.2 RF-driven neutron source configurations...........................................................

3.2.1 Axial Extraction Neutron Generator......................................................16

3.2.2 Co-axial Neutron Generator...................................................................18

3.3 Instrumental Neutron Activation Analysis experiments (INAA)..........................22

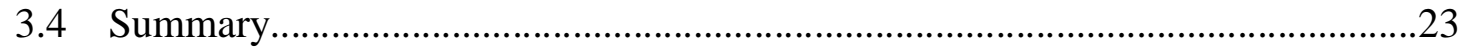

4 Methodology for computer simulations.............................................................................24

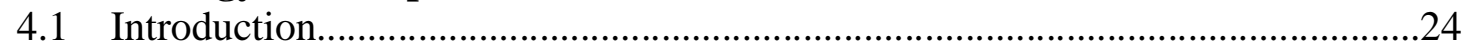




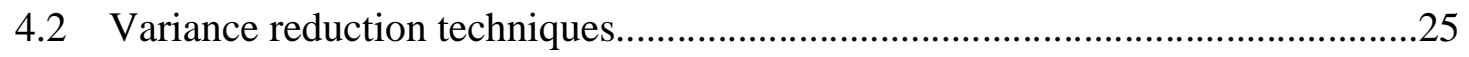

4.2.1 Geometry splitting and Russian Roulette............................................25

4.2.2 Point detector tally........................................................................26

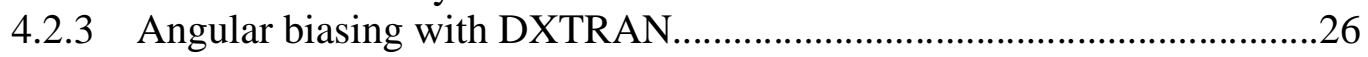

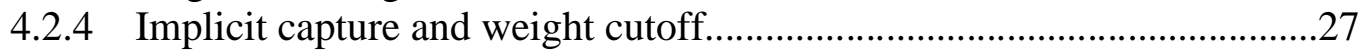

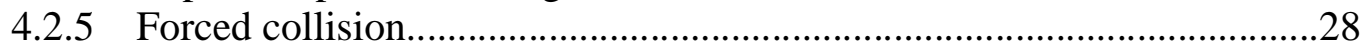

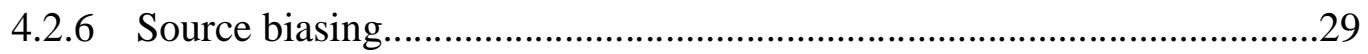

4.2.7 Neutron induced photon production biasing..........................................30

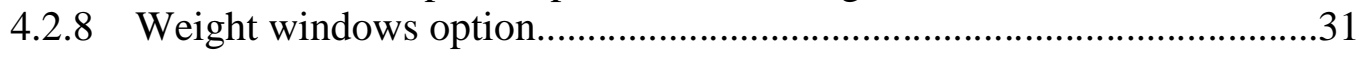

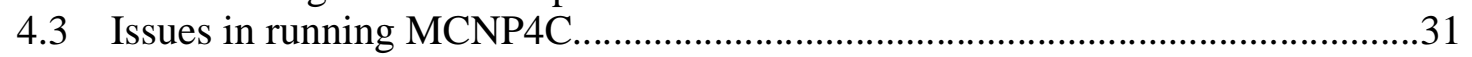

5 Neutron scattering experiments in condensed matter physics...............................34

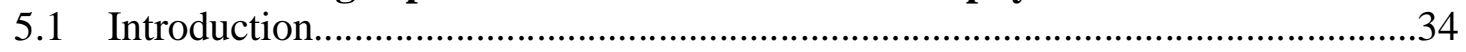

5.2 Thermal neutron beam for diffraction experiments.......................................36

5.2.1 Design parameters for thermal neutron scattering system and error

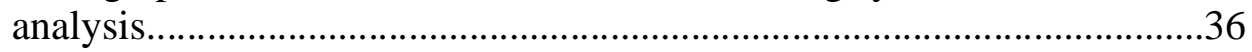

5.2.2 Computational model for a thermal neutron scattering facility...............40

5.2.3 Computational results for a thermal neutron scattering facility................44

5.3 Cold neutron source for Small Angle Neutron Scattering..................................51

5.4 Summary of fusion-based neutron sources for neutron scattering .......................55

6 Fission Multipliers for D-D/D-T Neutron Generators..................................................57

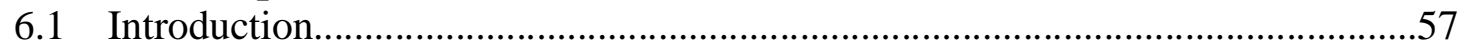

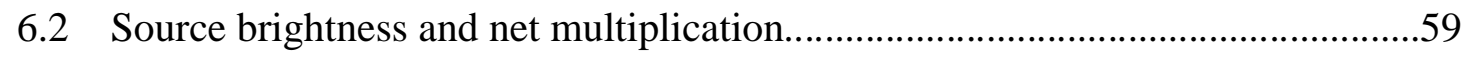

6.3 Designs of fission multipliers................................................................61

6.3.1 In-core irradiation - Positron production system................................62

6.3.2 Out-of-core irradiation - BNCT.......................................................64

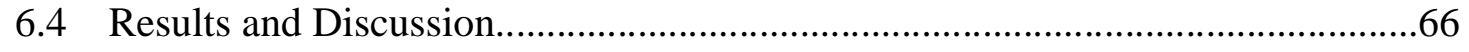

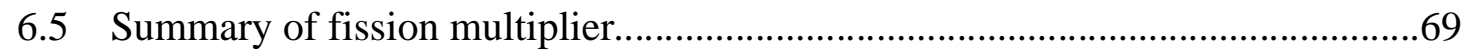

7 Other possible applications of fusion-based neutron generators...........................70

7.1 A review of neutron sources for contraband detection.....................................

7.1.1 Thermal Neutron Analysis (TNA)..................................................... 71

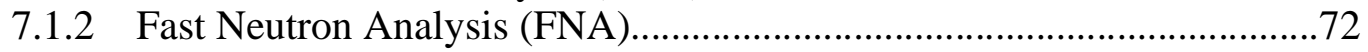

7.1.3 Associated Particle Imaging (API).................................................... 73

7.1.4 Pulsed Fast Neutron Spectroscopy (PFNS)..........................................74

7.1.5 Nuclear Resonance Radiography (NRR)...........................................74

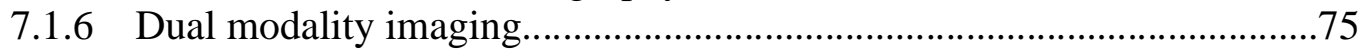

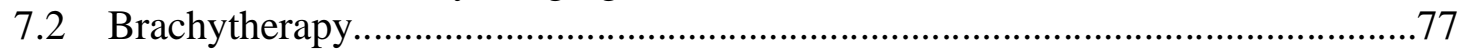

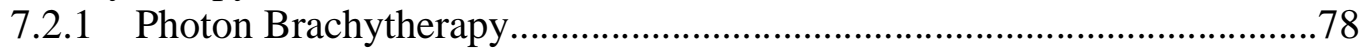

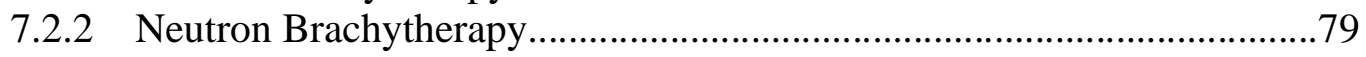

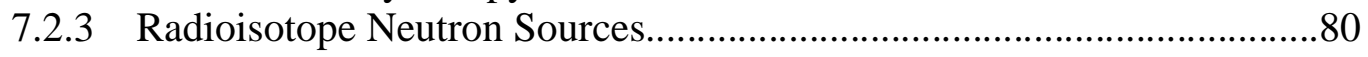

7.2.4 Fusion-based Neutron sources..............................................................80

7.2.5 Neutron dose profile, RBE and activation rates.................................82

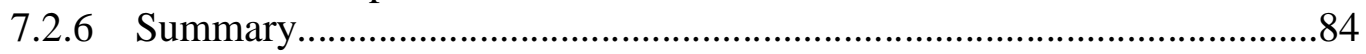




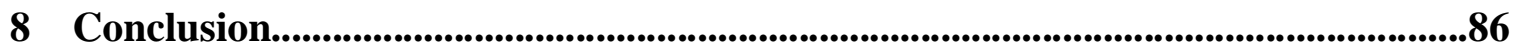

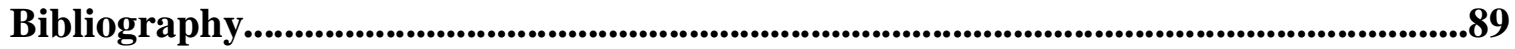

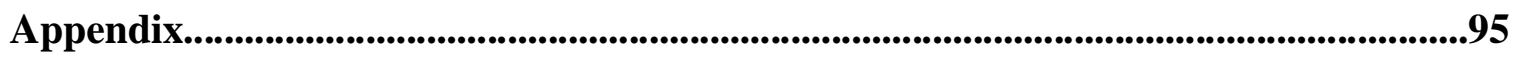

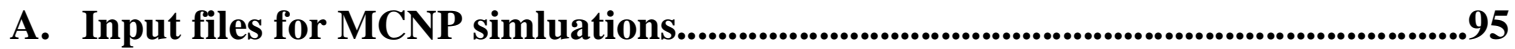




\section{List of Figures}

Figure 1.2.1 Neutron spectra due to D-D, D-T, and T-T reactions................................

Figure 1.2.2 Neutron production cross-section for D-D, D-T and D-T reactions............6

Figure 2.2.1 FiR-1 reactor for Boron Neutron Capture Therapy ...................................10

Figure 2.3.1 Spallation neutron source yield for different target materials....................12

Figure 2.3.2 Double differential cross section for $\mathrm{Pb}(\mathrm{n}, \mathrm{xn}) \mathrm{X}$ at $\mathrm{Ep}=0.8 \mathrm{GeV} \ldots \ldots \ldots \ldots . . .12$

Figure 3.2.1 Neutron generator testing facility......................................................16

Figure 3.2.2 The axial extraction neutron generator.................................................. 17

Figure 3.2.3 The minimum operating pressure of the axial extraction neutron generator ion source at various strength of the axial magnetic field and CW low

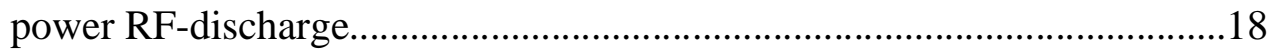

Figure 3.2.4 Co-axial neutron generator............................................................... 19

Figure 3.2.5 Ion beam dynamics simulation using IGUN...........................................20

Figure 3.2.6 Temperature distribution at the target for a beam current of $0.8 \mathrm{~mA}$ and an

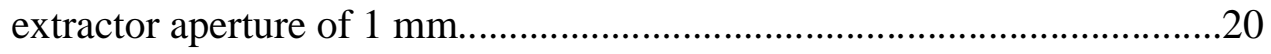

Figure 3.2.7 Neutron yield history with different operating voltage and beam current. 21

Figure 3.3.1 A $\gamma$ spectrum obtained from the prototype coaxial D-D neutron generator

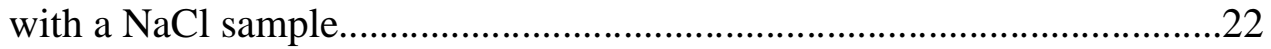

Figure 5.1.1 A two-dimensional representation of Bragg's law.....................................35 
Figure 5.2.1 A schematic diagram showing the relationship between the detector panel

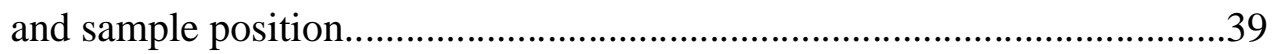

Figure 5.2.2 Geometry of thermal neutron scattering model in MCNPX......................41

Figure 5.2.3 Resolution at $\mathrm{d}=18.04 \AA$ at $2 \theta=20^{\circ}$ (ion beam width $\left.=50 \mu \mathrm{s}\right) \ldots \ldots \ldots \ldots . . .45$

Figure 5.2.4 Resolution at $\mathrm{d}=12.5 \AA$ at $2 \theta=20^{\circ}($ ion beam width $=50 \mu \mathrm{s}) \ldots \ldots \ldots \ldots \ldots . . . .45$

Figure 5.2.5 Resolution at $\mathrm{d}=8.62 \AA$ at $2 \theta=20^{\circ}($ ion beam width $=50 \mu \mathrm{s}) \ldots \ldots \ldots \ldots \ldots . . . . .46$

Figure 5.2.6 Resolution at $\mathrm{d}=1.97 \AA$ at $2 \theta=20^{\circ}$ (ion beam width $\left.=50 \mu \mathrm{s}\right) \ldots \ldots \ldots \ldots \ldots . . . .47$

Figure 5.2.7 Resolution at $\mathrm{d}=1.97 \AA$ at $2 \theta=20^{\circ}($ ion beam width $=0 \mathrm{~s}) \ldots \ldots \ldots \ldots \ldots \ldots . . . . . .48$

Figure 5.2.8 Resolution at $\mathrm{d}=2.85 \AA$ at $2 \theta=20^{\circ}$ (ion beam width $\left.=50 \mu \mathrm{s}\right) \ldots \ldots \ldots \ldots \ldots . . . .48$

Figure 5.2.9 Resolution at $\mathrm{d}=4.12 \AA$ at $2 \theta=20^{\circ}($ ion beam width $=50 \mu \mathrm{s}) \ldots \ldots \ldots \ldots \ldots . . . .49$

Figure 5.2.10 Resolution at $\mathrm{d}=0.76 \AA$ at $2 \theta=150^{\circ}$ (ion beam width $\left.=50 \mu \mathrm{s}\right) \ldots \ldots \ldots \ldots . . . .50$

Figure 5.2.11 Resolution at $\mathrm{d}=3.3 \AA$ at $2 \theta=150^{\circ}$ (ion beam width $=50 \mu \mathrm{s}$ ) ...............51

Figure 5.3.1 Moderator brightness curve for a D-T cold neutron source.......................52

Figure 5.3.2 Be and $\mathrm{C}$ neutron reaction cross sections from ENDF60..........................53

Figure 6.3.1 A D-T driven fission multiplier showing a Cd cap surrounded by a small

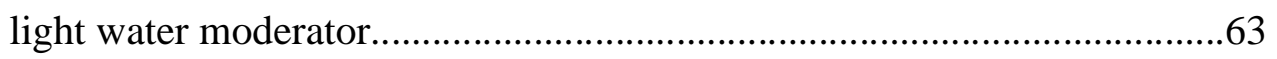

Figure 6.3.2 A cylindrical fuel assembly for the D-T fission multiplier.......................64

Figure 6.3.3 A D-D neutron driven fission multiplier with metal alloy fuel dispersed in

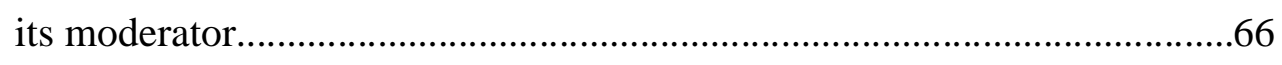

Figure 6.4.1 Epithermal neutron flux at the beam port exit of the BNCT system (Fission multiplier vs. D-D neutron generator) ...............................................68

Figure 7.2.1 A HDR 252Cf neutron brachytherapy treatment system..........................81 
Figure 7.2.2 A fusion-based neutron generator for brachytherapy ..............................82

Figure 7.2.3 Multiple neutron generators mounted on a single station.........................82

Figure 7.2.4 Dose Profile due to a point D-D neutron source.......................................83 


\section{List of Tables}

Table 5.1.1 Properties of neutrons for neutron scattering experiments.........................35

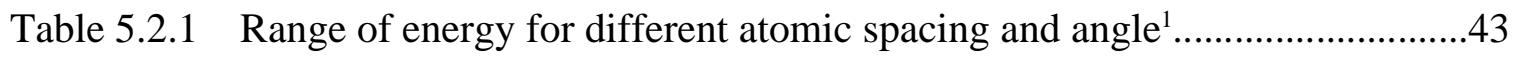

Table 6.3.1 Specification for the fuel and clad used in D-T driven fission multiplier...62

Table 6.3.2 Physical properties of U-10Mo alloy aluminum matrix dispersion fuel......65

Table 7.1.1 Some common explosives and their compositions..................................71

Table 7.2.1 Neutron induced charge particles in major tissue elements........................83 


\section{Acknowledgements}

I would like to thank the members of my dissertation committee Professor J. Vujic, Professor Ka-Ngo Leung, Professor Per F. Peterson, and Professor Hans-Rudolf Wenk for their scientific advice, help and support during this project. I would also like to express gratitude to all the members of the Plasma and Ion Source Technology Group for their scientific and their technical assistance. This work has been supported by the U.S. Department of Energy under contract number DE-AC03-76SF00098. 


\section{Chapter 1}

\section{Introduction}

\subsection{Applications of Neutrons}

Neutrons have a wide variety of applications related to different disciplines. For example, in material science engineers use fast neutrons to study the material properties

of the first wall of fusion reactor under long-term irradiation. Archaeologists and geologists use fast neutrons to irradiate samples of rocks for geochronological dating (e.g. Ar-Ar dating and fission track dating). In analytical chemistry, neutron activation analysis techniques such as Instrumental Neutron Activation Analysis (INAA), Radiochemical Neutron Activation Analysis (RNAA), Prompt Gamma Neutron Activation Analysis (PGNAA) and Chemical Neutron Activation Analysis (CNAA), are utilized to determine the trace elements in samples. In aerospace industry, neutron radiography is one of the Non-Destructive Evaluation (NDE) methods being used to detect corrosion, cracks, impact damage, foreign objects and flaws in manufacturing. In airports and at border crossing, neutron based non-intrusive inspection techniques are deployed to stop the smuggling of drugs, explosive, nuclear material and other contrabands. In hospitals, 
radioisotopes are used in radiotherapy, imaging and as pain relievers. Many of these radioisotopes are produced in nuclear reactors by neutron capture or other neutron induced reactions. Fast neutrons have been used as the primary radiation for brachytherapy, while epithermal neutrons have been applied to treat glioblastoma multiforme, a deep-seated brain tumor. In basic science research, physicists, chemists, material scientists, and biologists use neutron diffraction techniques to seek solutions to a wide variety of scientific challenges. For instance, biologists use cold neutrons to study protein crystallography and chemists have been using neutron scattering to obtain basic knowledge about microstructures in oils and creams. Furthermore, physicists use positron beams to detect lattice defects. Two common methods for positron production are radioactive decay of radioisotopes made in nuclear reactors and pair production due to absorption of gamma radiation emitted in a neutron capture reaction. A variety of neutron sources are available for these applications. These neutron sources are reviewed in Chapter 2. In this study, we focus on the fusion-based neutron sources.

\subsection{Properties of fusion-based neutron generators}

The fusion reactions considered in this study are induced by the collision of accelerated deuterium and/or tritium ions. Deuterium is a stable isotope of hydrogen with one neutron and one proton (i.e. $\mathrm{D}={ }^{2} \mathrm{H}$ ) while tritium is a radioactive isotope of hydrogen with two neutrons and one proton (i.e. $\mathrm{D}={ }^{3} \mathrm{H}$ ). ${ }^{3} \mathrm{He}$ is produced in the D-D reaction, ${ }^{2} \mathrm{H}(\mathrm{d}, \mathrm{n})$. ${ }^{4} \mathrm{He}$ is produced in the $\mathrm{D}-\mathrm{T}$ reaction, ${ }^{3} \mathrm{H}(\mathrm{d}, \mathrm{n})$. The most common neutron generators available commercially are based on D-D or D-T fusion reaction. These commercial fusion-based neutron generators have a low cost and short operational lifetime. There are two major factors contributing to the short operational lifetime. One of 
these factors is the outgassing of deuterium or tritium from the target due to the high power density delivered to the target by the beam. Another factor is the sputtering of target atoms. The neutron yields of commercial fusion-based D-D/D-T neutron generators also decrease as a function of lifetime due to the depletion of deuterium or tritium atoms on the target. Furthermore, most commercial D-D/D-T neutron generators have ion sources that produce plasmas with a low yield of monatomic species. The scientists in the Plasma \& Ion Source Technology (PIST) Group of the Ion Beam Technologies (IBT) program under the Accelerator and Fusion Research Division (AFRD) at Lawrence Berkeley National Laboratory (LBNL) realized that their expertise in making ion sources could address many of these problems. As a result, a new generation of D-D/D-T fusionbased neutron generators is currently being developed by IBT.

\subsubsection{Monoenergetic fusion-based neutron sources}

The D-D and D-T reactions produce monoenergetic neutrons with energy of 2.45 $\mathrm{MeV}$ and $14 \mathrm{MeV}$ respectively when the kinetic energy of $\mathrm{D}^{+}$and/or $\mathrm{T}^{+}$ions is comparatively small. ${ }^{2} \mathrm{H}(\mathrm{d}, \mathrm{n})^{3} \mathrm{He}$ reaction has a Q-value of $+3.270 \mathrm{MeV}$ and a threshold energy of 4.45 MeV for its break up reaction $\mathrm{D}(\mathrm{d}, \mathrm{np}) \mathrm{D}$.[1] In some applications such as fast neutron reaction cross section measurement or cargo inspection, D-D neutrons are produced by high energy accelerator to generate monoenergetic neutron beam with energy range from 1.64 to $7.75 \mathrm{MeV}$.[1] ${ }^{3} \mathrm{H}(\mathrm{d}, \mathrm{n}){ }^{4} \mathrm{He}$ reaction has a Q-value of +17.590 $\mathrm{MeV}$ and threshold energies of 3.71 and $4.92 \mathrm{MeV}$ for its break up reactions $\mathrm{T}(\mathrm{d}, \mathrm{np}) \mathrm{T}$ and $\mathrm{T}(\mathrm{d}, 2 \mathrm{n})^{3} \mathrm{He}$.[1] A monoenergetic neutron beam with energy ranging from 11.74 to 20.5 can be produced from the D-T reactions using a high energy accelerator.[1] The neutron generators being developed at LBNL use a low energy accelerator and are 
therefore not designed for those particular applications that required monoenergetic fast neutrons with energy other than $2.45 \mathrm{MeV}$ and $14 \mathrm{MeV}$. Examples of the $14 \mathrm{MeV}$ fast neutron applications are fusion reaction material studies, stockpile stewardship radiochemistry, neutron radiography, Ar-Ar dating and fission track dating. The 2.45 $\mathrm{MeV}$ or $14 \mathrm{MeV}$ neutrons can be slowed down or thermalized to epithermal, thermal or cold neutrons. These moderated neutrons have a wide variety of applications as mentioned in the introduction section of this thesis.

The new D-D/D-T neutron generators being developed have several characteristics that differentiate them from commercial fusion-based neutron generators. They have a much longer lifetime due to the self-loading target design. The target is a thin layer of titanium explosive-bonded on aluminum. The aluminum backing is cooled by water to an optimum operating temperature. The optimum operating temperature is presently determined by experiments because the temperature of $\mathrm{Ti}$ is not the only parameter that determines the diffusivity of deuterium or tritium atoms in Ti. For examples, impurities in the material have large effects on the diffusivity. Without the precise knowledge of the impurities in the material, experiments must be carried out to obtain an optimum operating temperature.

The deuterium or tritium atoms are loaded into the titanium layer by means of ion-implantation. The deuterium or tritium target atoms are thus self-replenished by the operation of the neutron generator. The lifetime is limited by the sputtering rate of $\mathrm{Ti}$ atoms on the target. On the contrary, commercial neutron generators have $\mathrm{TiT}_{2}$ or $\mathrm{TiD}_{2}$ target. The hydride form of titanium decomposes into metallic titanium at $\sim 500{ }^{\circ} \mathrm{C}$. The commercial pre-loaded $\mathrm{TiT}_{2}$ or $\mathrm{TiD}_{2}$ targets have a very short lifetime because they are 
made of brittle materials. In addition, the new designs also offer a higher neutron yield than commercial neutron generators. A discussion of the prototype designs is presented in Section 1.3.

\subsubsection{Fusion-based white neutron source}

A white neutron source emits neutrons with a continuous energy distribution. A white neutron spectrum can be obtained with a T-T fusion-based neutron generator. Unlike, D-T or D-D reactions, the products of T-T reaction are two neutrons and one ${ }^{4} \mathrm{He}$ atom. The Q-value for this reaction is $11.31 \mathrm{MeV}$ and is shared by the two neutrons and the ${ }^{4} \mathrm{He}$ atom. The white neutron spectrum (Figure 1.2.1) can be calculated nonrelativistically in the three-body kinetic phase space.[2] The T-T source neutron spectrum

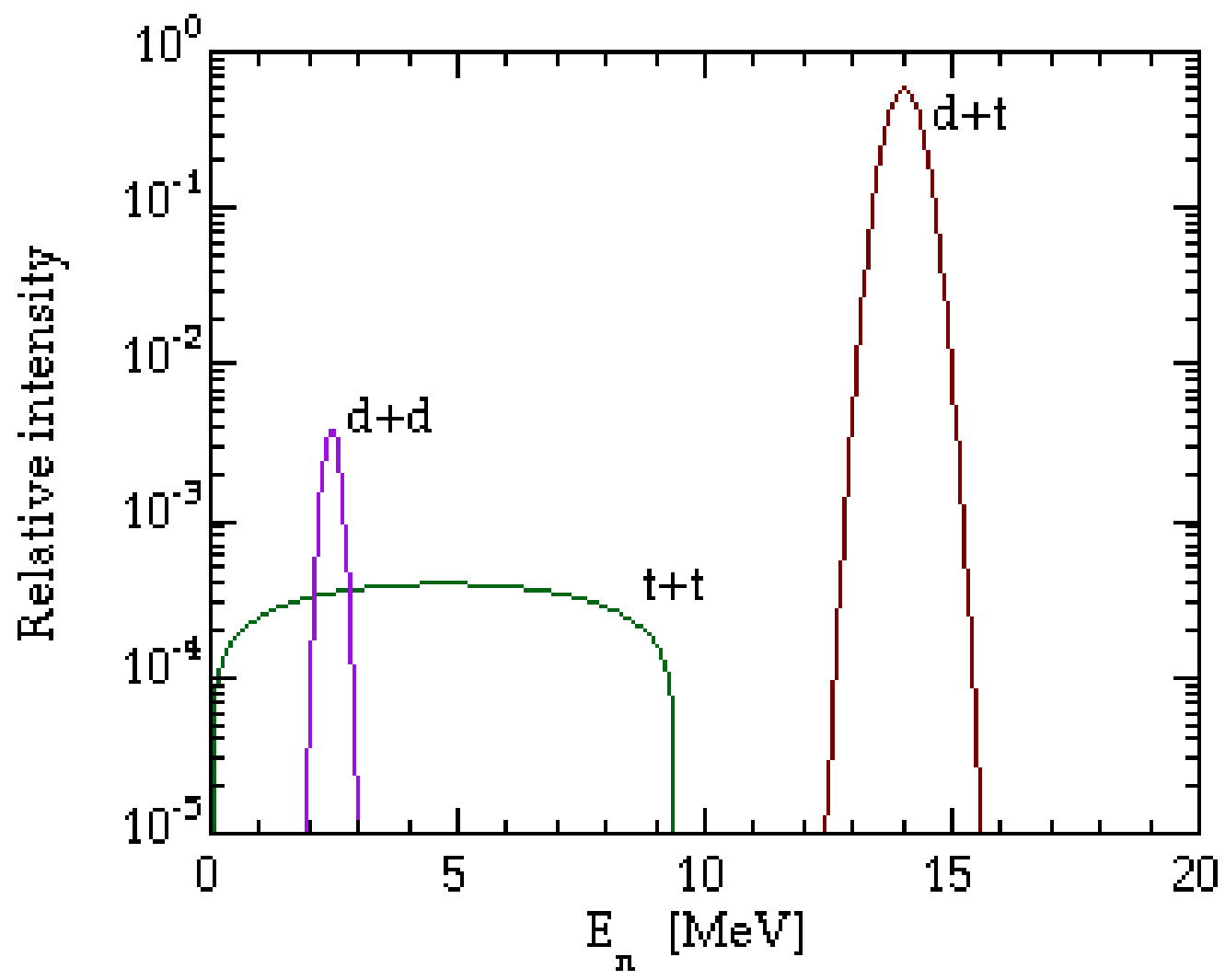

Figure 1.2.1 Neutron spectra due to D-D, D-T, and T-T reactions 
in Figure 1.2.1 can be expressed by Equation 1.1. The ${ }^{3} \mathrm{H}(\mathrm{t}, 2 \mathrm{n})^{4} \mathrm{He}$ reaction has a crosssection approximately the same as ${ }^{2} \mathrm{H}(\mathrm{d}, \mathrm{n})^{3} \mathrm{He}$ reaction as shown in Figure 1.2.2.[3]

$$
\frac{d N}{d E_{n}}=\frac{4 \pi \sqrt{E_{n}\left(\frac{5}{6} Q-E_{n}\right)}}{25}
$$

where $\quad N$ is the number of neutrons.

$E_{n}$ is the neutron energy.

$Q$ is the $\mathrm{Q}$-value of the T-T reaction.

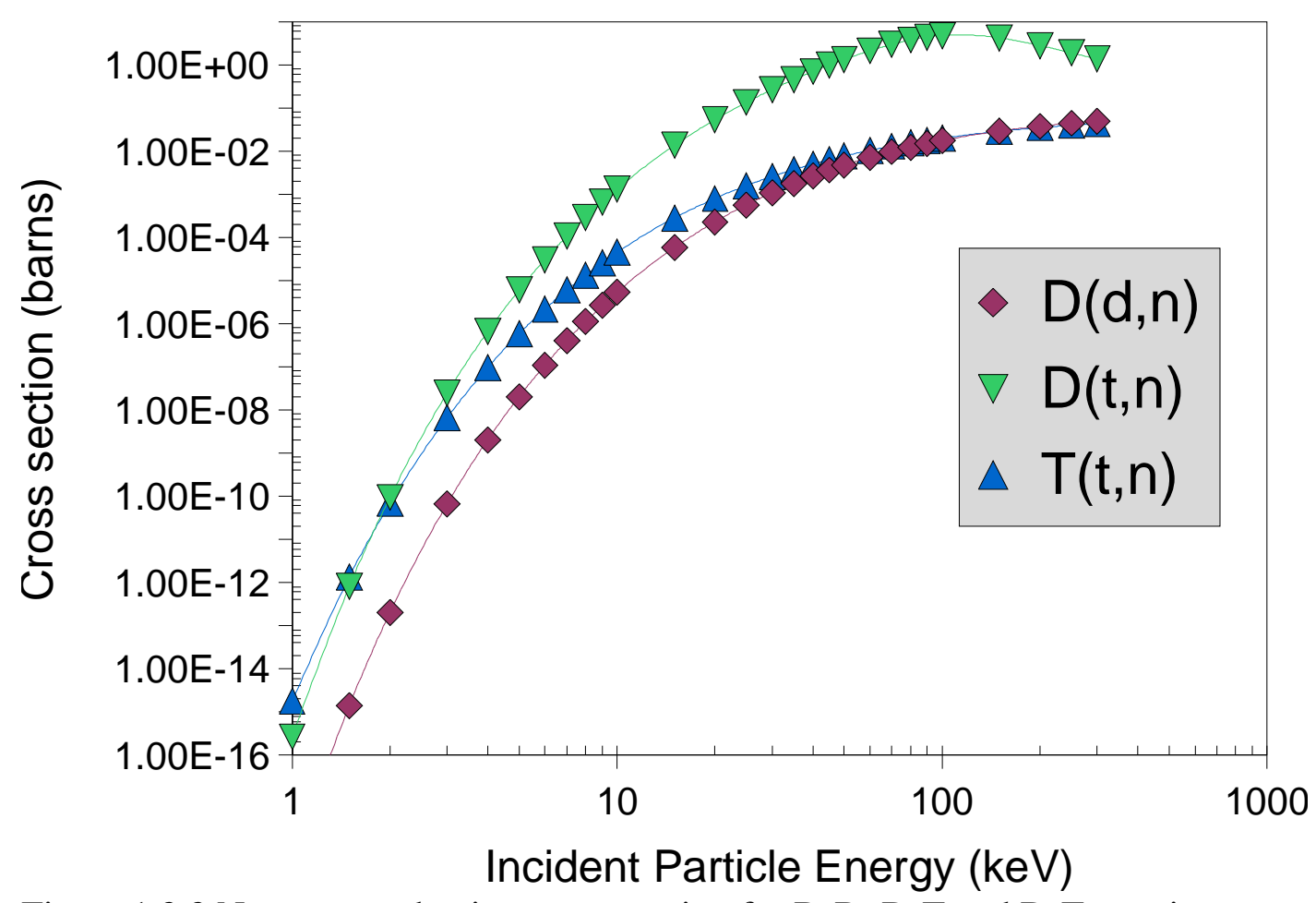

Figure 1.2.2 Neutron production cross-section for D-D, D-T and D-T reactions

The total neutron yield of a T-T neutron generator is approximately twice as much as the yield of a D-D neutron generator because the T-T reaction emits two neutrons in its fusion reaction. The white neutron spectrum is especially important for fast neutron transmission spectroscopy. 


\subsection{Scope of thesis research}

Because the new designs of fusion-based neutron generators have the potential of producing orders of magnitude higher neutron flux than conventional fusion-based neutron generators, a series of studies are carried out to evaluate the feasibility of using these neutron generators in applications that require expensive neutron sources such as fission reactors, spallation neutron sources, etc. This study will concentrate on optimizing the neutronics design of each system to make the neutron flux of these fusion-based neutron generators comparable to that of other expensive neutron sources. 


\section{Chapter 2}

\section{Survey of other neutron sources and their properties}

\subsection{Introduction}

According to the statistics from International Atomic Energy Agency's (IAEA) Research Reactor DataBase (RRDB), only twenty-eight research reactors owned by the universities in the United States remain operational today.[4] There are forty-eight university operated research reactors decommissioned or shutdown in the past sixty years. Twenty-four research reactors remained operational and are owned by Department of Energy (DOE), private companies, national laboratories, Department of Defense (DOD) laboratories and military.[4] The university research reactors serve as neutron sources for different researches and educational tools. As more research reactors are getting decommissioned and shutdown, other neutron sources are being developed and being used for research. In this section, the properties of several commonly available neutron sources are discussed. 


\subsection{Fission reactors}

Research fission reactors were the major neutron sources available for university research. However, many university research reactors were shutdown and decommissioned. The research reactor fuel is usually made of $\mathrm{U}-\mathrm{Al}$ alloy, $\mathrm{UO}_{2}$ in polyethylene, $\mathrm{U}_{3} \mathrm{Si}_{2} \mathrm{Al}_{\mathrm{x}}, \mathrm{UO}_{2}$, and/or $\mathrm{UAl}_{\mathrm{x}}$. The reactor types are ARGONAUT, HOMOG(S), POOL, TANK, TANK in POOL, and TRIGA. Out of these reactor types, the more common ones are TRIGA and POOL.[4] A TRIGA reactor is a pulsed reactor but it only means that the power of a TRIGA reactor can be increased to a much higher level during the pulse. There is only one pulsed reactor called IBR-2 that is used for neutron scattering experiments in Russia.[5] IBR-2 is a fast reactor and its core is fueled with seven plutonium dioxide fuel elements. It has a thermal neutron pulse width of $320 \mu \mathrm{s}$ and is operating at $5 \mathrm{~Hz}$.[6]

The time average neutron flux of a research reactor has a range of $10^{6}$ to $10^{15}$ $\mathrm{n} / \mathrm{cm}^{2} \mathrm{~s}$ depending on its size and power. The neutron flux from a fission reactor is proportional to the power of the system. Because neutron flux is a function of neutron density in a system, a research reactor usually has a small core size. In order to get critical with small core size, some research reactors use highly enriched uranium fuel (HEU). Efforts have been made to convert some of these HEU research reactors to low enriched uranium by using high uranium density fuel such as $\mathrm{U}-\mathrm{Al}, \mathrm{U}_{3} \mathrm{Si}_{2} \mathrm{Al}_{\mathrm{x}}$ and $\mathrm{UAl}_{\mathrm{x}}$. These research reactors also have large reflectors and may have high neutron leakage in the chamber where the neutrons are being extracted. Thus, the neutron flux in a reactor is limited by its size and cooling capability. As a result, there is a limit in the neutron flux that a reactor can produce. 
Many research reactors have a thermalized neutron energy spectrum because they have a hydrogenous moderator in order to make the core compact. The neutrons in a reactor initially have a fission spectrum and they are thermalized if the reactor is a thermal reactor. Some applications require epithermal neutrons rather than thermal neutrons. Figure 2.2.1 shows an example of using a TRIGA research reactor where the neutrons are extracted from its graphite reflector for Boron Neutron Capture Therapy (BNCT). The fast neutrons are extracted from a reflector next to the core. The thermal neutrons are shielded with neutron absorber while the fast neutrons are slowed down by another moderator to produce epithermal neutrons. Another approach of obtaining an external epithermal neutron beam is to convert the thermal neutrons with a fission

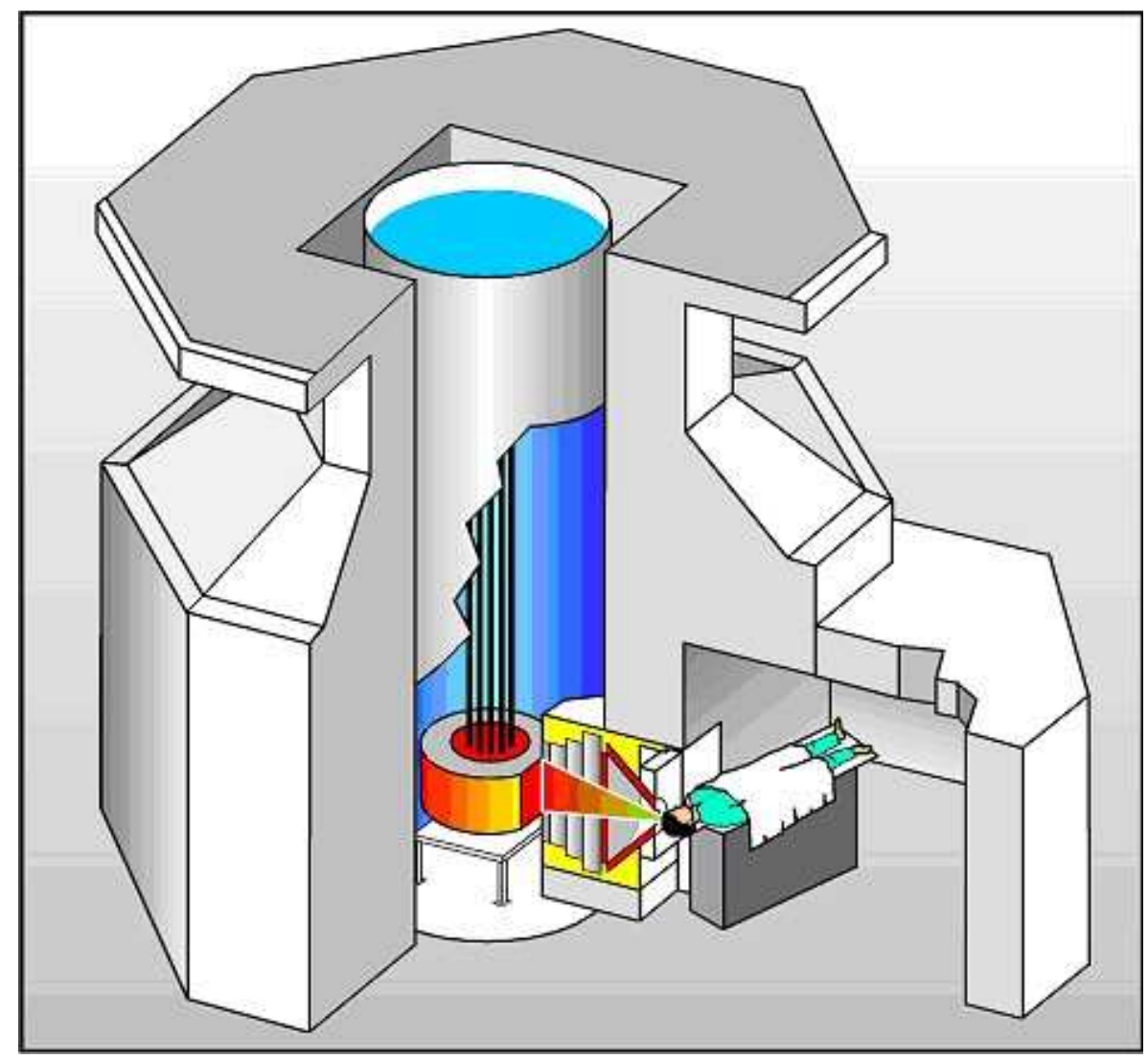

Figure 2.2.1 FiR-1 reactor for Boron Neutron Capture Therapy 
converter plate outside the core. The fission neutrons induced by the thermal neutrons can be moderated to the desired energy.[7]

\subsection{Spallation neutron sources}

A spallation neutron source is an accelerator based pulsed neutron source. When a proton is accelerated to hundreds of $\mathrm{MeV}$ or $\mathrm{GeV}$, it is energetic enough to knock out some neutrons of a heavy atomic nucleus such as mercury, lead, tungsten, or uranium. This nuclear reaction is called spallation. As a proton bombards a heavy atomic nucleus, the bombarded nucleus is left in its excited state and additional neutrons are emitted. There are 20 to 30 neutrons expelled in a spallation process. On average, approximately 2.5 fission neutrons are generated per fission and one fission neutron is used to sustain the chain reaction in a fission reactors. The number of neutrons available is $\sim 1.5$ per fission and each fission generates $\sim 200 \mathrm{MeV}$. Therefore, spallation neutron sources produce more neutrons per unit energy in the system when compared to fission reactors. Figure 2.3.1 shows the calculated (from a general-purpose transport code called RST\&T) and measured (from NESSI experiment) neutron yield of different spallation targets.[8] Degtyarev shows that neutron spectrum in a spallation process has a very long high energy tail. These high energy neutrons make up a significant amount of the total neutron population. They are emitted in the forward direction as shown in Figure 2.3.2 when an $800 \mathrm{MeV}$ proton beam is hitting on a lead target. These high energy neutrons $(>100 \mathrm{MeV})$ require a thick shielding to stop them. Because they are usually undesirable for most applications, neutron beams are usually not extracted in parallel to the incident proton beam line. Instead, neutron beam lines are perpendicular to the proton beam line. 


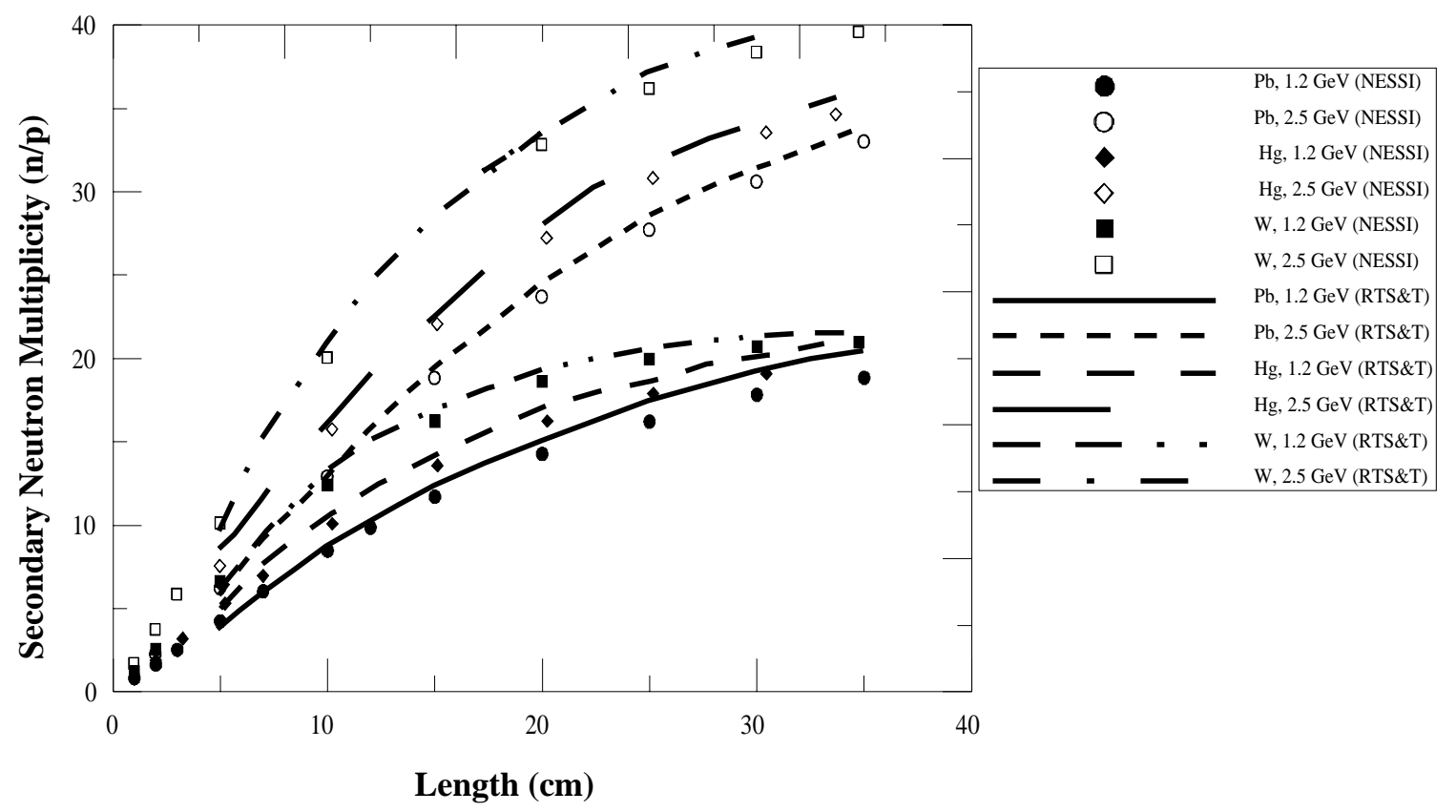

Figure 2.3.1 Spallation neutron source yield for different target materials

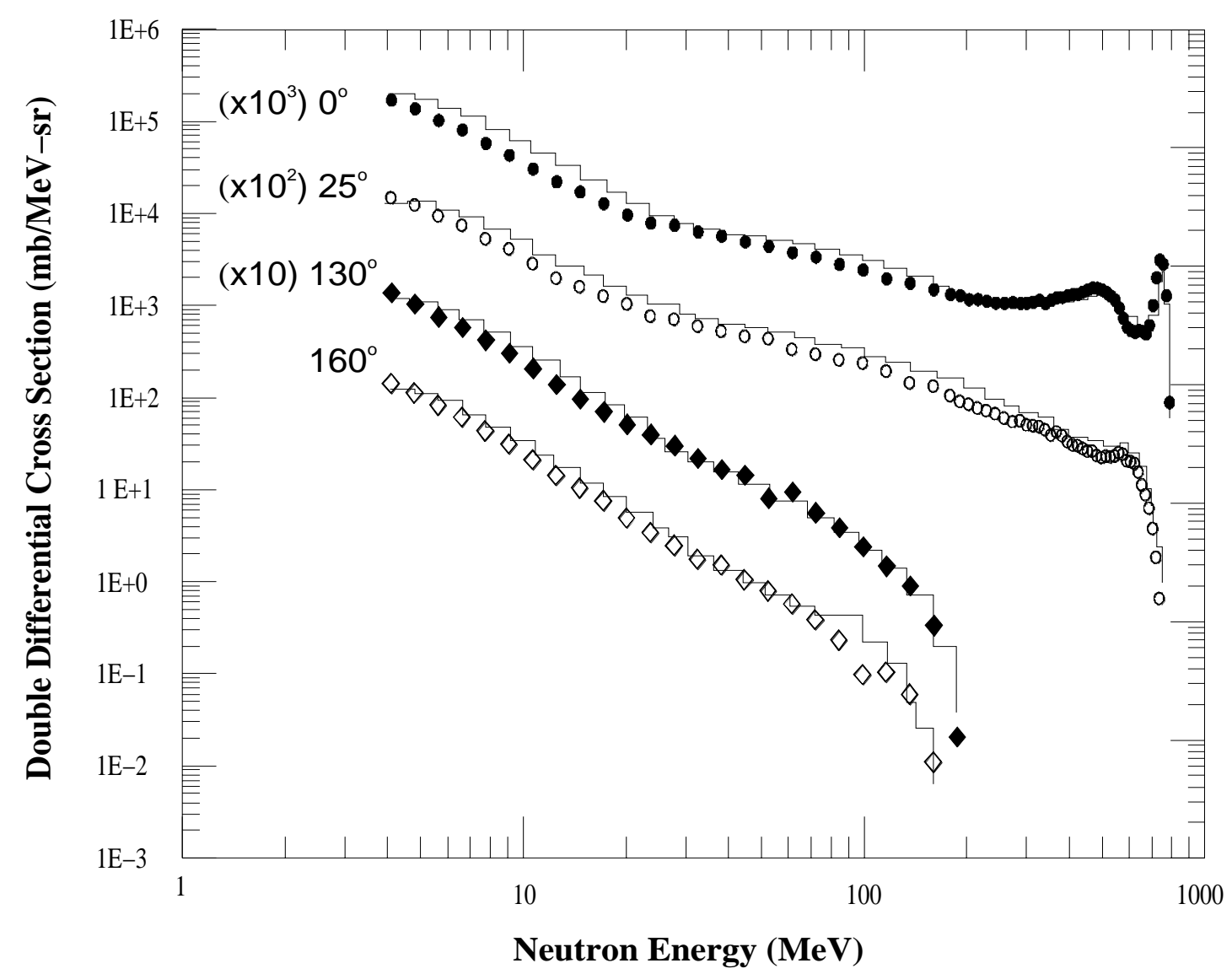

Figure 2.3.2 Double differential cross section for $\mathrm{Pb}(\mathrm{n}, \mathrm{xn}) \mathrm{X}$ at $\mathrm{E}_{\mathrm{p}}=0.8 \mathrm{GeV}$ 
A modern spallation target is usually split into two parts and moderators are placed around the space between the split targets. This kind of configuration is called flux-trap/split target transmission/transmission design and is implemented in the shortpulse spallation target at the Lujan Center of the Los Alamos Neutron Science Center (LANSCE) from 1985 to 1997.[9] LANSCE was an acronym for the Lujan Center before 1995.

\subsection{Charge particles induced neutron sources}

There are other ways to produce neutrons with charge particle interaction beside spallation. Some examples of these reactions are ${ }^{3} \mathrm{H}(\mathrm{p}, \mathrm{n}){ }^{3} \mathrm{He},{ }^{7} \mathrm{Li}(\mathrm{p}, \mathrm{n}){ }^{7} \mathrm{Be},{ }^{9} \mathrm{Be}(\mathrm{p}, \mathrm{n}){ }^{9} \mathrm{~B}$, and ${ }^{9} \mathrm{Be}(\mathrm{d}, \mathrm{n}){ }^{10} \mathrm{~B}$. The Q-values for these four reactions are $-0.763 \mathrm{MeV},-1.644 \mathrm{MeV}[1]$, $-1.852 \mathrm{MeV}[10]$, and +4.36 MeV[11]. Although the neutron yields of these reactions are not as high as the spallation process, these neutron production reactions have their own particular applications. For instance, $\mathrm{p}$ - $\mathrm{T}$ neutron source is used for fast neutron crosssection measurement in the range of 0.3 to $7.6 \mathrm{MeV}$, while $\mathrm{p}$-Li neutron source is a monoenergetic neutron source in the range 0.12 to $0.6 \mathrm{MeV}$. One common characteristic of these neutron generators is that they all require high energy $(>\mathrm{MeV})$ accelerator to get a reasonable neutron yield. For example, in order to increase the neutron yield of d-Be reactions, the deuteron energy is usually above the threshold energy of several manybody reaction channels such as $(\mathrm{d}, 2 \mathrm{n}),(\mathrm{d}, \mathrm{pn})$ and $(\mathrm{d}, \mathrm{p} 2 \mathrm{n}) \cdot[11]$

\subsection{Radioisotope neutron sources}

There are several radioisotopes that emit spontaneous fission neutrons, of which

${ }^{252} \mathrm{Cf}$ is the most common. The spontaneous fission neutrons have a fission neutron 
spectrum. ${ }^{252} \mathrm{Cf}$ neutron source has very high source neutron intensity and is passively cooled. Source neutron intensity is defined as the yield per unit volume. One milligram of ${ }^{252} \mathrm{Cf}$ has a yield of $2.3 \times 10^{9} \mathrm{n} / \mathrm{s}$. Another type of radioisotope neutron sources is usually made of an alpha emitting radioisotope and a light element that has a high $(\alpha, n)$ reaction cross-section. The $(\alpha, n)$ radioisotope neutron sources have much lower neutron intensity. For example, one milligram of ${ }^{241} \mathrm{Am}$ in an AmBe source has a yield of $6.8 \times 10^{3} \mathrm{n} / \mathrm{s}$.

\subsection{Summary}

The number of university research reactors is diminishing in the United States. Spallation neutron source is becoming more important because of its high neutron yield. The spallation neutron source has the highest yield in the forward direction. It has a very high energy tail at small angle. Because these high energy (>20 MeV) neutrons are not utilized in some applications, the new fusion-based neutron generator may be able to produce comparable neutron flux for certain applications. While neutron sources such as p-Be and p-Li can be applied to some of these applications, they require a high energy accelerator that is more expensive than the fusion-based neutron generators. For these reasons, the new fusion-based neutron generators and their applications are the topic of this research. 


\section{Chapter 3}

\section{New fusion-based neutron generator designs}

\subsection{Introduction}

Several types of fusion-based neutron generators are currently under development and testing at LBNL. A prototype neutron facility for Neutron Activation Analysis (NAA) and other neutron experiments was built to demonstrate the concept of the new fusion-based neutron generators driven by high current and low voltage accelerators. A prototype coaxial D-D neutron generator with a total beam current of $>1 \mathrm{~mA}$ is currently producing $>10^{9} \mathrm{n} / \mathrm{s}$. This neutron generator is located in Building 52 of the Lawrence Berkeley National Laboratory. It is shielded with polyethylene and lead. Figure 3.2.1 shows the CAD drawing of this neutron generator testing facility.

\subsection{RF-driven neutron source configurations}

There are three new fusion-based neutron generator designs currently under testing by the PIST group scientists: (a) axial, (b) coaxial, and (c) toroidal. Two common 


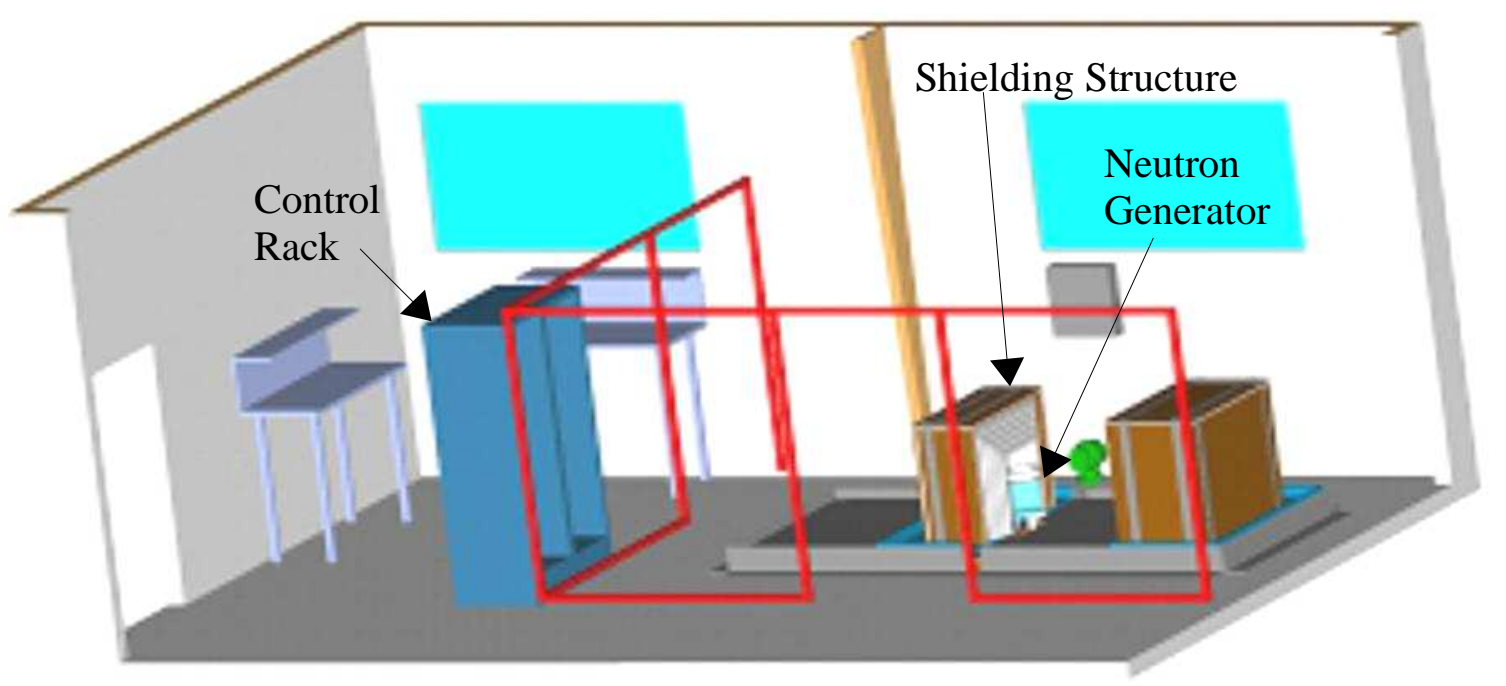

Figure 3.2.1 Neutron generator testing facility

features in these designs are the RF-driven ion sources and the explosive-bonded titanium-on-aluminum targets. The plasma is generated in the ion source using RF induction discharge. A $13.5 \mathrm{MHz}$ RF power supply is used to drive plasma in either continuous working (cw) or pulsed operation mode. The RF system consists of a RF power supply and a matching network, which matches the impedance of the plasma, the antenna and the coaxial transmission line. The RF discharge is shown to produce high plasma density and high atomic species fractions.[12] The explosive bonded titanium-onaluminum target material is developed and manufactured by ATLAS Technologies. The axial and co-axial neutron generators are discussed in the following sections.

\subsubsection{Axial Extraction Neutron Generator}

One of the axial designs currently under testing is approximately $40 \mathrm{~cm}$ in length and $15 \mathrm{~cm}$ in diameter. The ion source consists of a quartz-tube and an external antenna as shown in Figure 3.2.2. The deuterium gas is fed through the back plate of the ion source with a pressure read-out. The target is housed in an aluminum vacuum vessel and 


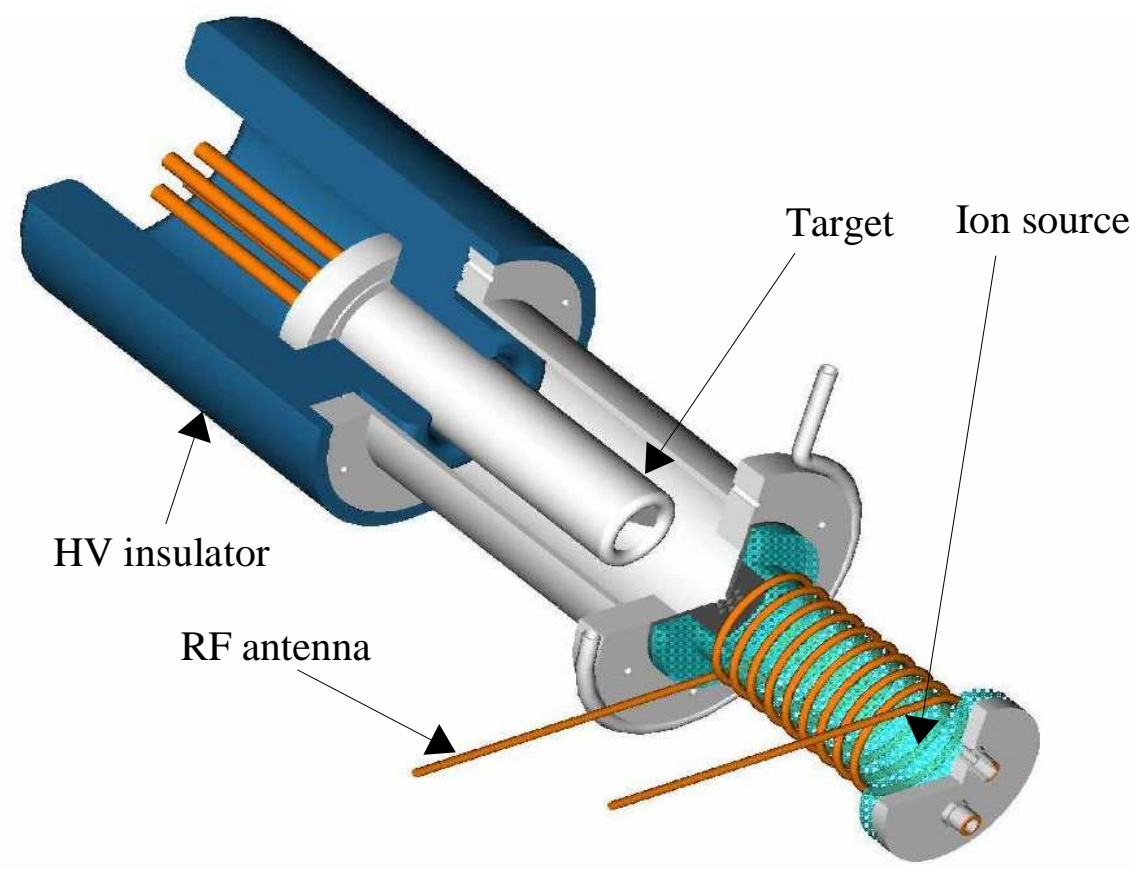

Figure 3.2.2 The axial extraction neutron generator

it is insulated from the ground potential with a high voltage (HV) insulator. The ion beam extraction hole has a $3 \mathrm{~mm}$ diameter. The ions are accelerated to the target with a potential of $100 \mathrm{kV}$. The plasma electrode is a molybdenum disk cooled by water.

A turbo molecular pump constantly evacuates the D-D neutron generator. If the neutron generator is modified to use tritium gas, the neutron generator has to operate in a sealed-tube condition. When the ion source is operated at low gas pressure, the RF amplifier is modulated to provide high-power pulses. Between the high power pulses, low-amplitude RF power is supplied to maintain a low-density plasma. In addition, an axial electron confining magnetic field is introduced to the discharge chamber by using the RF induction coil to carry a DC current. With a solenoid current of 140 A, a minimum operational plasma chamber pressure of $\sim 20 \mathrm{mTorr}$ is measured at the plasma chamber by using a barocell manometer (Figure 3.2.3). The ion confinement scheme is 


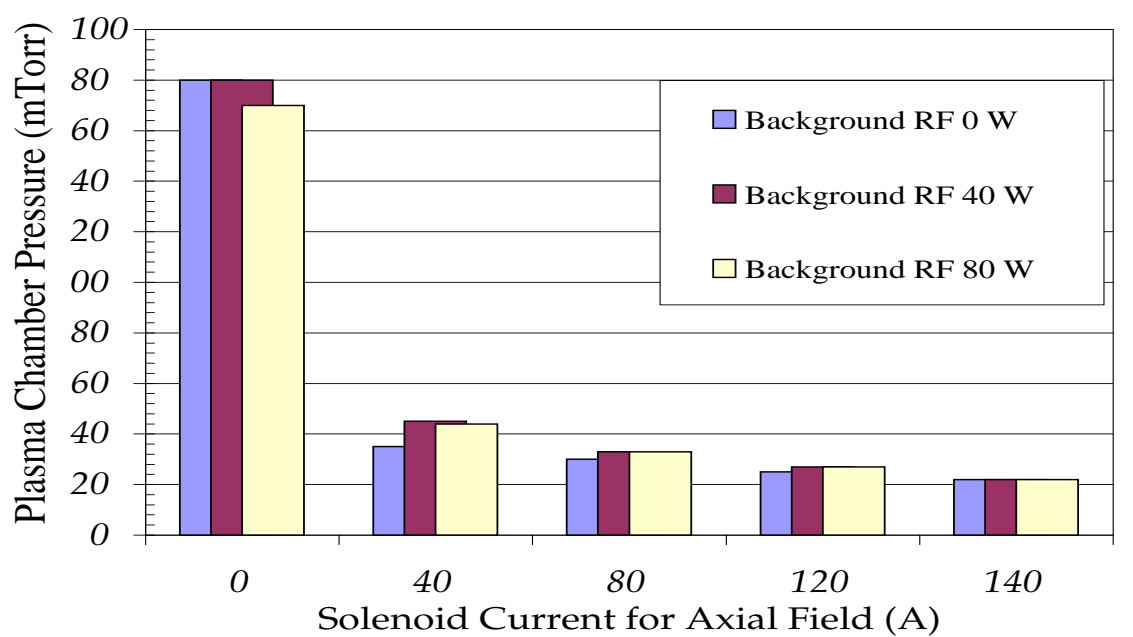

Figure 3.2.3 The minimum operating pressure of the axial extraction neutron generator ion source at various strength of the axial magnetic field and CW low power RF-discharge

shown to work experimentally. The experiment also shows that the low power CW RF discharge can lower the minimum pressure when there is no axial magnetic field. The time average neutron yield currently obtained with the prototype compact axial neutron generator is measured to be $10^{8} \mathrm{n} / \mathrm{s}$ at $10 \%$ duty factor.

\subsubsection{Co-axial Neutron Generator}

The co-axial neutron generator has been tested in the same neutron facility. The plasma is formed by utilizing $13.56 \mathrm{MHz}$ RF induction discharge. The ion source chamber is in the middle of the tube and surrounded by the target. The ions are extracted radially to the cylindrical shell target as shown in Figure 3.2.4. Therefore, the target area can be maximized in a given volume. The system is surrounded by a HV insulator cylinder made of Pyrex glass. This configuration protects the HV insulator from the sputtered target particles. The co-axial neutron generator currently being tested has a diameter of $30 \mathrm{~cm}$ and a height of $40 \mathrm{~cm}$. There are twenty-four extraction holes. Each 


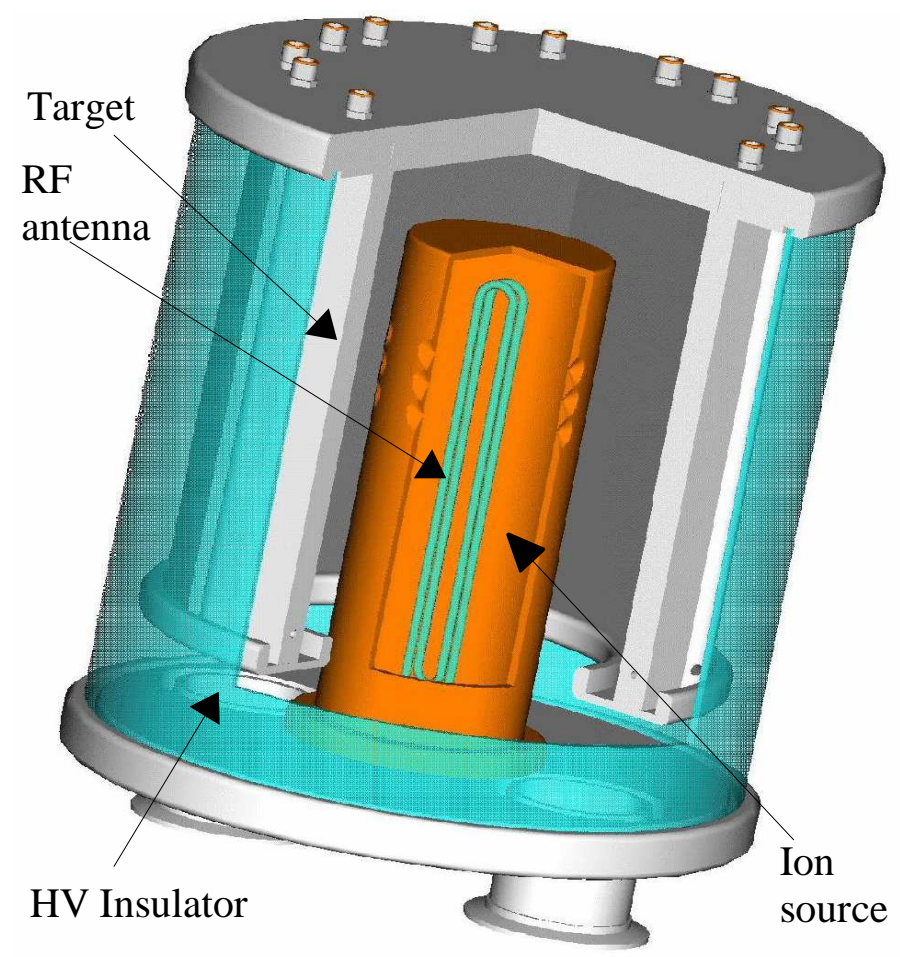

Figure 3.2.4 Co-axial neutron generator

extraction hole has a diameter of $1.5 \mathrm{~mm}$. The water-cooled target plates are $6.3 \mathrm{~cm}$ from the plasma chamber wall. A turbo-molecular pump is used to pump-evacuate the generator. The beam dynamic simulations were performed with IGUN ion beam extraction and ion trajectory simulation program. The temperature profile of the target is predicted by the ANSYS-modeling program with the beam spot size obtained from IGUN simulations.[13] Figure 3.2.5 shows the results of IGUN simulation of a $0.8 \mathrm{~mA}$ ion beam extracted from one of the extraction hole with an extractor aperture of $1 \mathrm{~mm}$ and a gap of $6.3 \mathrm{~cm}$. Figure 3.2.6 shows the temperature distribution at the target with that ion current. Experiments shows that the surface temperature at the beam spot reaches more than $800^{\circ} \mathrm{C}$ at which the neutron flux stays constant because the desorption rate of deuterium is equal to the product of the beam spot area and the ion beam current. The neutron yield of the co-axial neutron generator is measured to be $>10^{9} \mathrm{n} / \mathrm{s}$ during the experiment. The 


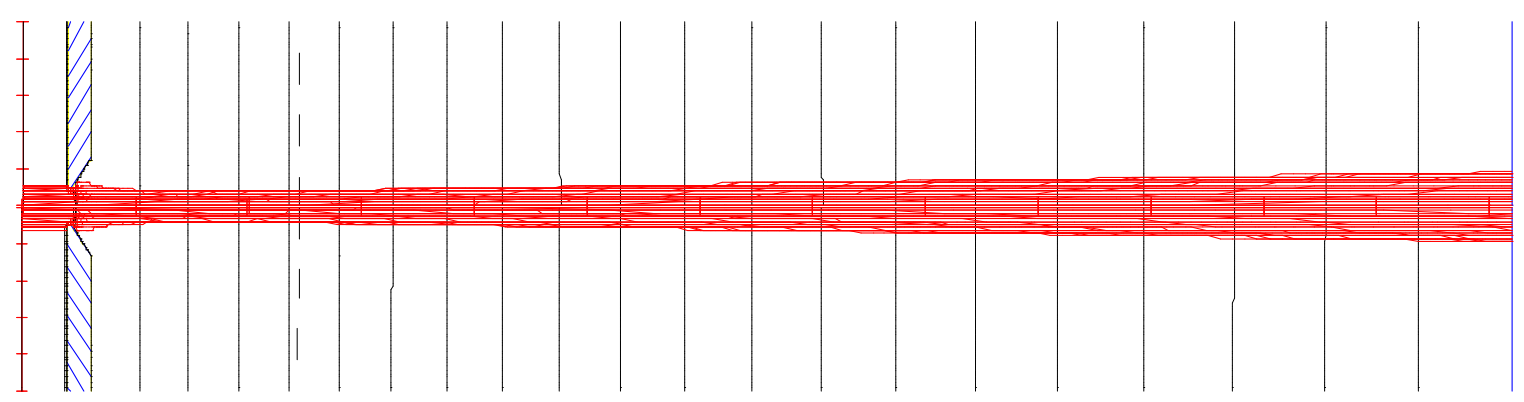

Figure 3.2.5 Ion beam dynamics simulation using IGUN

measurement was done with gold foil in a paraffin block enclosed by cadmium. It takes approximately one minute to reach this stable operation after a cold start. During the cold start, some of the implanted deuterium atoms diffuse to the back of the target and the concentration eventually saturates due to the impurities or lattice defects in the Ti target. As the concentration of deuterium atoms saturates inside the target, the deuterium atoms

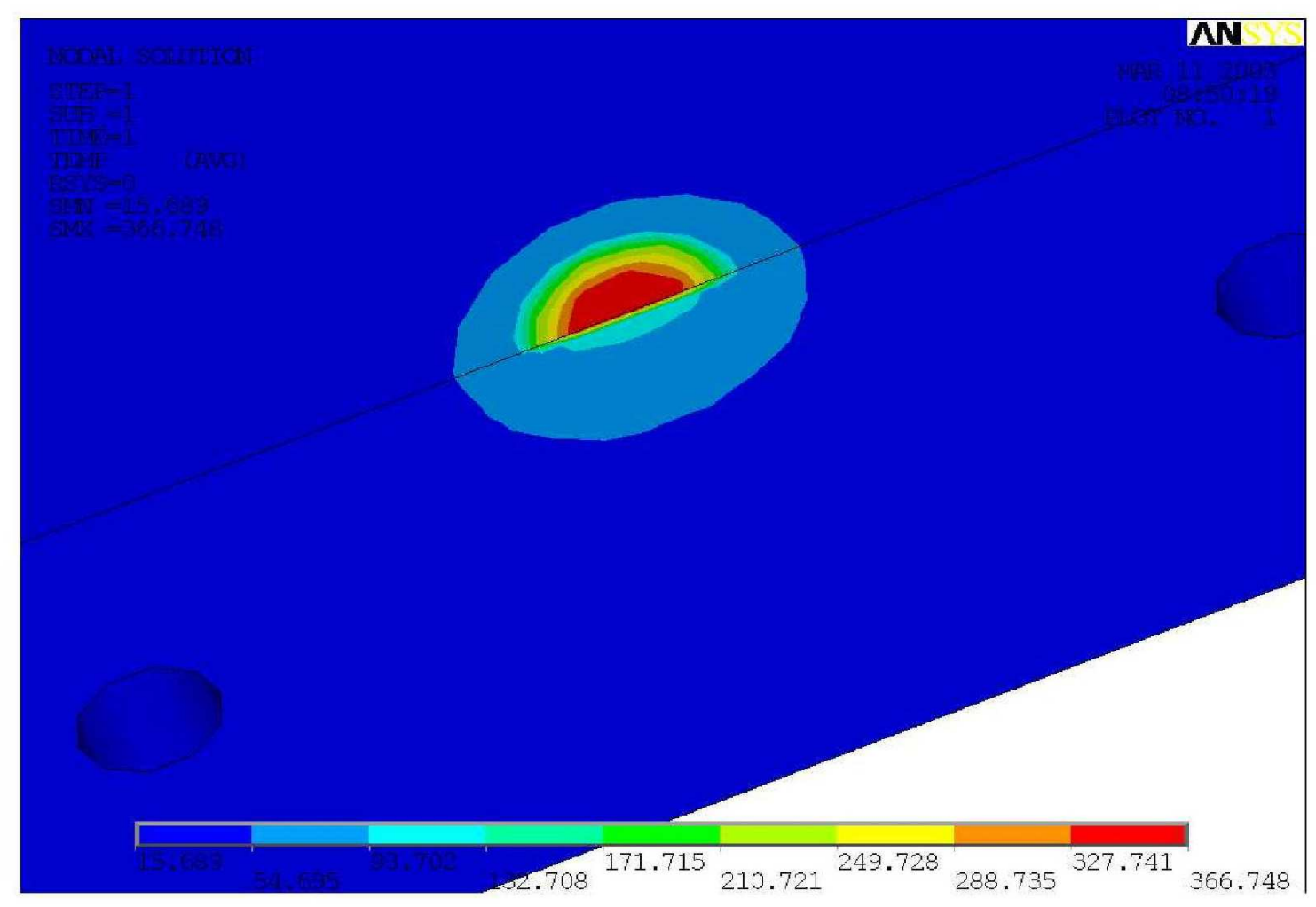

Figure 3.2.6 Temperature distribution at the target for a beam current of $0.8 \mathrm{~mA}$ and an extractor aperture of $1 \mathrm{~mm}$ 
start to re-combine at the surface of the target at the same rate of deuterium hitting on the target.

Figure 3.2.7 shows the neutron yield during the testing operation. The acceleration voltage was raised from $80 \mathrm{kV}$ to $100 \mathrm{kV}$ in two steps. The experiment shows that the target temperature reaches its critical temperature at a beam power of 2.4 $\mathrm{kW}$. When the beam power is reduced back to $2 \mathrm{~kW}$, the neutron yield stabilizes to the original value.

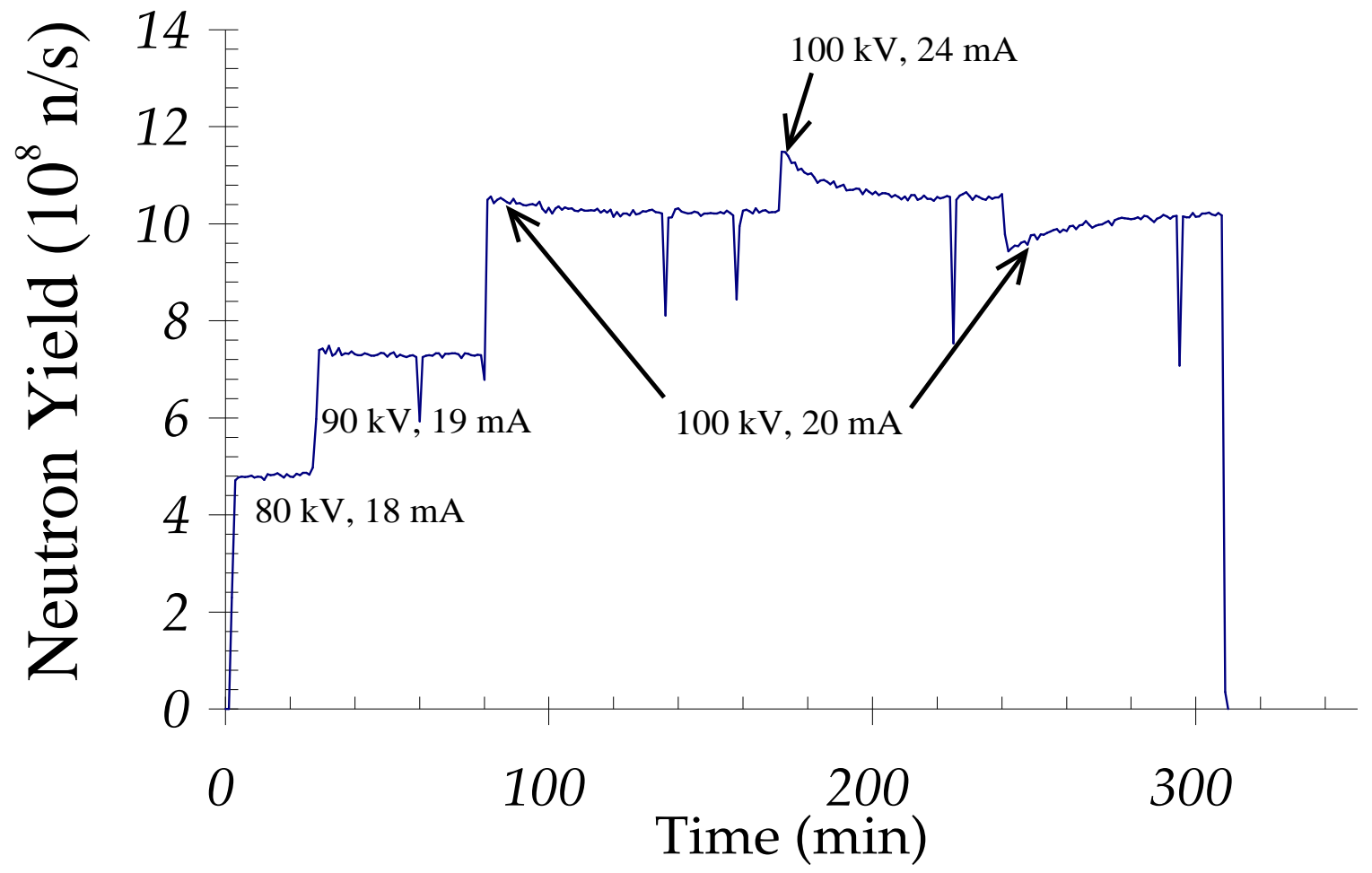

Figure 3.2.7 Neutron yield history with different operating voltage and beam current

The neutron yield of the current prototype co-axial D-D neutron generator is more than 10 times of a typical commercial D-D neutron generator during this experiment. Samples have been placed inside the shielding of the prototype D-D co-axial neutron generator as a simple irradiation demonstration. A brief discussion about these experiments is presented in the next section. 


\subsection{Instrumental Neutron Activation Analysis experiments (INAA)}

Currently, there is one high purity germanium (HPGe) detector available in the neutron generator testing facility for Instrumental Neutron Activation Analysis (INAA). Figure 3.3.1 shows one of the gamma spectra obtained with a reagent-grade $\mathrm{NaCl}$ sample irradiated with a neutron yield of $\sim 10^{9} \mathrm{n} / \mathrm{s}$ from the prototype coaxial D-D neutron generator. The detection limit was not determined for the current neutron generator because the flux of the prototype neutron generator is currently much lower than that of a research reactor. The polyethylene acts both as the moderator and the shielding. The

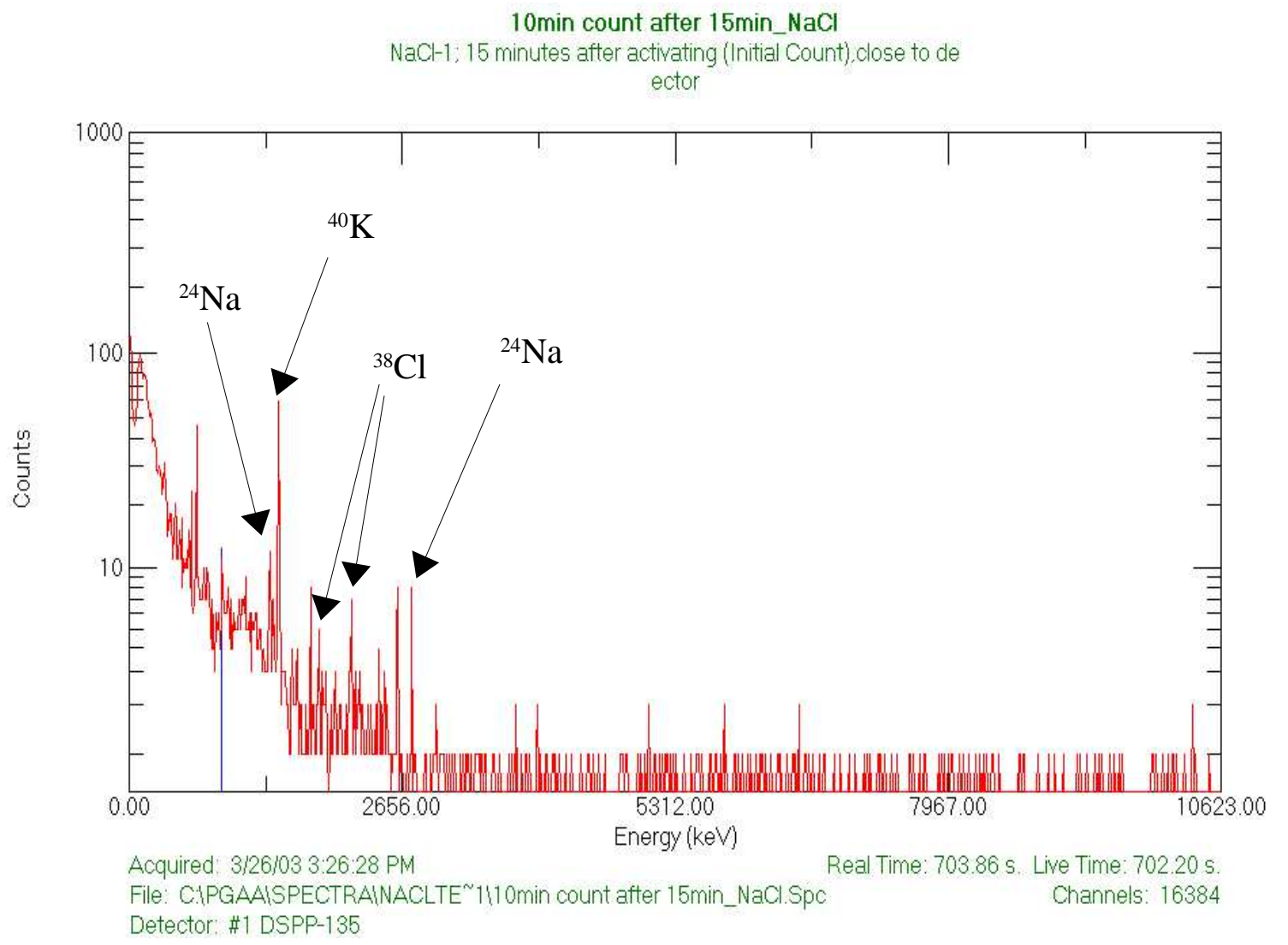

Figure 3.3.1 A $\gamma$ spectrum obtained from the prototype coaxial D-D neutron generator with a $\mathrm{NaCl}$ sample 
sample was placed three inches into the polyethylene. After the irradiation, the sample was counted by a High Purity Germanium (HPGe) detector.

\subsection{Summary}

Two types of the new D-D neutron generators are described in this chapter: the axial and co-axial types. The neutron yield is measured to be $>10^{9} \mathrm{n} / \mathrm{s}$ for the coaxial design and $>10^{8} \mathrm{n} / \mathrm{s}$ for the axial design. An INAA experiment was done by irradiating a $\mathrm{NaCl}$ sample with the prototype coaxial neutron generator. This simple INAA experiment demonstrates that the new fusion-based neutron generator is a promising tool for doing neutron experiments or educating students in neutron science. An upgraded version of the co-axial design is being reviewed and will be implemented in the near future. After the upgrade of the facility, more INAA experiments will be carried out as progress. A Prompt Gamma Neutron Activation Analysis (PGNAA) facility will also be considered when adequate funding becomes available. A PGNAA facility will require more sophisticated equipments and instrumentation (e.g. a supermirror neutron bender and a HPGe detector with Compton-suppression system). 


\section{Chapter 4}

\section{Methodology for computer simulations}

\subsection{Introduction}

In order to analyze various designs of D-D/D-T neutron generators and their applications, we have to rely on computer simulations of particle transport for many experimental set-ups. A neutronics computer code called Monte Carlo N-Particle (MCNP) [14] version 4C is used to simulate neutron transport in all computer simulations in this dissertation. MCNP is chosen because it can handle many complicated geometries and it uses the continuous energy neutron reaction cross sections. MCNP4C is capable of handling three types of particles - neutron, photons and electrons. It also has many variance reduction techniques that allow the user to simulate different problems within a reasonable amount of computational time. Furthermore, the code has several multiprocessing versions. It can be compiled with Parallel Virtual Machine (PVM), a distributed memory multitasking software that allows a heterogeneous collection of UNIX or Windows computers be connected together by a network and used as a single 
large parallel computer. The other multiprocessing versions of MCNP are shared memory multitasking (i.e. OpenMP) and Message Passing Interface (MPI). (NOTES: MPI is another distributed memory multitasking software. The MPI version of MCNP is only available in MCNP5 or MCNPX2.5.C.)

\subsection{Variance reduction techniques}

Several variance reduction techniques are used in the simulations whenever it is appropriate. The documentation for variance reduction techniques in MCNP manual does not provide simple examples for a novice user. A novice user can learn these variance reduction techniques by attending a MCNP Workshop or reading other references [15]. A discussion of the variance reduction techniques useful for the works in this dissertation is presented in the following subsections.

\subsubsection{Geometry splitting and Russian Roulette}

The most common variance reduction technique for MCNP is geometry splitting and Russian roulette (i.e. Cell importance card) because they have simple of syntaxes in an input file. The user only needs to assign a cell importance card to each cell. When a neutron crosses from a less important cell to a more important cell, the neutron is split. When a neutron crosses from a more important cell to a less important cell, a Russian roulette is played. If the neutron survives, its weight is raised. Otherwise, the particle is killed. This technique allows the code to spend more time in sampling the important regions in geometry. This technique reduces the variance but increases the time per history. 


\subsubsection{Point detector tally}

Point detector tally is a fast way to measure photon flux or neutron flux in a small volume far away from the source. For every collision in the system, a deterministic estimate of flux due to that particle collision is performed at the point detector position. This is a useful feature in scoping out the optimum parameters in a design. However, it may not provide the exact information we want to obtain. For instance, it is more useful to obtain the pulse height tally for gammas coming from contraband. A pulse height tally shows what is actually measured by a physical detector, while a point detector tally measures the actual photon flux. It is necessary to use point detector tally for scoping studies because pulse height tally is not compatible with all variance reduction techniques except source biasing. Source biasing and neutron induced photon production are discussed in later sections.

\subsubsection{Angular biasing with DXTRAN}

The DXTRAN technique is very similar to a point detector tally. It biases the scattering directions to a small region that is inadequately sampled. A DXTRAN sphere is defined to enclose this small region of interest. Upon particle collision outside the DXTRAN sphere, a DXTRAN particle is created with the weight adjusted for biasing the scattering angle. While a pseudo particle of a point detector is scattered toward the point detector, a DXTRAN particle is scattered to a randomly sampled position on the surface of the DXTRAN sphere. As a DXTRAN particle is transported deterministically without collision to the surface of the DXTRAN sphere, its weight is exponentially decreased by its optical path. The non-DXTRAN particle will continue to be sampled in the normal 
way until it enters the DXTRAN sphere. When a non-DXTRAN particle tries to enter the DXTRAN sphere, it is killed in order to balance the particle weight contribute to the cells inside the DXTRAN sphere. The DXTRAN technique is very useful in the simulation of moderator designs for thermal neutron scattering. It allows the user to estimate the resolution for such system. A more detailed discussion on resolution estimation is given in the later section covering neutron scattering.

\subsubsection{Implicit capture and weight cutoff}

For neutron transport in MCNP, implicit capture is the default setting. It is suitable for most neutron transport problems. When a neutron collides with a nuclide, the neutron always survives with a new weight.

$$
w_{\text {new }}=w_{o} \cdot\left(1-\frac{\sigma_{a i}}{\sigma_{t i}}\right)
$$

where $\quad w_{\text {now }}=$ new weight after collision

$$
\begin{aligned}
& w_{\mathrm{o}}=\text { weight before collision } \\
& \sigma_{\mathrm{ai}}=\text { microscopic absorption cross section for nuclide } \mathrm{i} \\
& \sigma_{\mathrm{ti}}=\text { total microscopic cross section for nuclide } \mathrm{i}
\end{aligned}
$$

Russian roulette is played if a particle's weight drops below a user-specified weight cutoff. The particle is either killed by Russian roulette in weight cutoff or its weight is increased to a user-specified level. This technique can be applied to most problems effectively. However, it is not suitable for simulation involving cold neutrons because the ratio of the microscopic absorption cross section and the total microscopic cross section for cold neutron is very close to unity. The cold neutron's new weight after collision is almost zero, so the weight cutoff will prevent the cold neutrons to continue 
with their history. There are two ways to turn off the weight cutoff: forced collision (to be discussed in the next session) and analog capture. Because implicit capture will increase the time per history, analog capture will reduce the time per history. Weight cutoff assumes that cold neutron weight is too low to be worth transporting. Using the implicit capture option will not reduce the variance of cold neutron tallies. As a result, analog capture is preferred for cold neutron problems as it reduces computer time per history. In this case, an over sampling in regions outside the cold moderator is assumed and there is no time bins for the tallies.

\subsubsection{Forced collision}

As mentioned in previous section, weight cutoff is favorable in cold neutron transport problems. Although the computer time per history is reduced when analog capture is used in the simulation, it will still take a long time to obtain a reasonable variance for neutron tallies with small time bins, because a neutron pulse structure requires more sampling in regions that are far away from the cold moderator. Cold neutrons have a short mean free path in all materials. Most cold neutrons are generated in a cold moderator with a high hydrogen contents so the variance for cold neutron tallies can be reduced by sampling the cold moderator more frequently. However, cold neutron pulse usually has a long tail because the neutrons spend a long time slowing down to thermal energy in the reflector and moderator before hitting the cold moderator. In order to reduce the variance of the signals in a neutron pulse shape, sampling in the regions far away from the cold moderator becomes more important. Applying forced collision technique in the cold neutron problems has two benefits. Forced collision technique can be applied to sample collisions in optically thin cells (e.g. Cold moderator) by turning 
weight cutoff in these particular cells. The other benefit is that it increases the sampling of high energy neutrons, which have a long mean free path in a relatively small cold moderator. A neutron entering a forced collision cell is split into an uncollided track and a collided track. The uncollided track will traverse the cell with an expected weight.

$$
w_{u}=w e^{-\Sigma l}
$$

where

$$
\begin{aligned}
& w_{u}=\text { uncollided weight } \\
& w=\text { incoming weight } \\
& \Sigma=\text { total macroscopic cross section } \\
& l=\text { distance across the cell }
\end{aligned}
$$

The collided track carried a weight of $\left(1-w_{u}\right)$ and a collision site is sampled within l. Because weight cutoff is turned off in forced collision cells, the computer time per history can be extremely long if there are too many adjacent forced collision cells. The computer time per history can be controlled either by using a reasonable number of forced collision cells or by disabling implicit capture. The user should choose to reduce the number of forced collision cells and use the default implicit capture if other regions in the system are not sampled frequently enough.

\subsubsection{Source biasing}

The angular distribution and energy distribution can be biased in MCNP. The code can sample the regions of interest more frequently with the appropriate weight. For the D-D/D-T fusion-based neutron generators, the source neutron energy is discrete. Therefore, it is not necessary to bias energy distribution in these problems. However, biasing the angular distribution is useful when the object being irradiated is located in a 
particular direction from the source. For instance, biasing the angular distribution of a neutron source in an explosive detection system can reduce the variance of the neutron induced photon tallies.

\subsubsection{Neutron induced photon production biasing}

In the effort of modeling a contraband detection system, photon production biasing becomes the most important technique because a pulse height tally forbids the usage of variance reductions techniques other than source biasing. A pulse height tally calculates the difference between the products of energy and weight of a particle entering a cell and leaving a cell. Therefore, the weight of a particle cannot be changed from its source weight whenever pulse height tallies are concerned. If the particle is a positron, the energy will be kinetic energy plus $1.022 \mathrm{MeV}$. Photons are created with a weight of $W_{p}$.

$$
W_{p}=\frac{W_{n} \sigma_{\gamma}}{\sigma_{T}}
$$

where $\quad W_{s}=$ neutron weight

$$
\begin{aligned}
& \sigma_{\gamma}=\text { photon production cross section } \\
& \sigma_{T}=\text { total neutron cross section }
\end{aligned}
$$

In MCNP, there is a minimum weight $\left(W_{i}^{\text {min }}\right)$ for a photon produced in cell $i$. The default value for this minimum weight is set to the weight of source neutron. If the ratio of minimum weight and source neutron weight times the ratio of neutron importance in cell $i$ and neutron importance in a source cell is less than unity (i.e. $W_{p} / W_{i}^{\min } \times I_{i} / I_{s}<1$ ), Russian roulette is played. If it is greater than unity, one or more photons are generated. The number of neutrons created is $N p$, 


$$
N_{p}=\frac{W_{p} I_{i}}{W_{i}^{\min } I_{s} \times 5}+1, \quad N \leq 10
$$

Each photon has a weight of $W_{p} / N_{p}$. In a general problem, the default value can avoid too many photons generated. However, in situation similar to a contraband detection system, one would like to sample more frequently in the cell of interest (i.e. Cell with explosive). The $W_{i}^{\text {min }}$ is set to a small number in the input file with a PWT card so that more photons are generated in each photon production reaction.

\subsubsection{Weight windows option}

Weight windows option is not used in all calculations done for this dissertation because it generally has a long computer time per history. This is a problem for running MCNP with PVM. The code tends to terminate the spawned processes prematurely whenever a process spends too much time. Official patch and unofficial patch had been applied to the code but they are unable to resolve the problem. Another reason of not using weight windows option is that automatic weight window generation takes a long time.

\subsection{Issues in running MCNP4C}

Although MCNP is widely used in the nuclear industry, its user community is relatively small compared to other popular computer codes' user communities. Furthermore, MCNP4C supports almost any platform with standard FORTRAN 77 and C compilers. It is inevitable to have problems with some features on certain platforms. The problems encountered in our simulations are summarized in this section. 
Large file is a term referring to file with a size greater than 2 GB. The large file support is desirable because a user may want to generate a large surface source for subsequent simulation. The size of surface source file can often grow over 2 GB. All platforms, except PC's, supported by MCNP4C are 64-bit systems. Personal computers with a 32-bit processor used to have a filesize limitation of $2 \mathrm{~GB}$. The computers used to obtain the results presented in this dissertation are Intel x86 PC's running Solaris 8 and FreeBSD 4.1 and Linux 2.4.18. These are 32-bit machines with a "64-bit clean" Operating System. In other words, there are some extensions in the OS to deal with many limitations in a 32-bit processor. However, these extension or features are sometimes disabled by default for performance reasons. For example, a Linux user needs to compile a program and link it to libraries with large file support and enable largefile support in its kernel. In Solaris 8 for $x 86$, the FORTRAN compiler is no longer a support product for SUN. It does not support largefile although the Solaris 8 does.

Another problem of MCNP4C encountered is premature PVM process termination in its PVM version. MCNP4C will terminate its spawned PVM subtasks when it takes too much time to rendezvous particles from them or when too many secondary particles are banked for subsequent transport calculation. There are several patches used to increase the size of secondary particle banks. However, they do not resolve this problem. The solution to this problem is to indirectly adjust the number of particles rendezvoused by MCNP each time, by changing the frequency with which MCNP dumps its data back into its data files. MCNPX 2.4 has a better load balancing and higher fault tolerance and does not have the problem mentioned above. Also, there is a bug forbidding MCNP4C to write a surface source in its PVM version. There is an 
official patch from MCNP official website to fix this bug [16]. It is worth to mention here that MCNP5, the next version of MCNP to be released in 2003, has angular flux tally, and MPI support. It is claimed that the MPI version has a higher fault tolerance in its multiprocessing part of the code. 


\section{Chapter 5}

\section{Neutron scattering experiments in condensed matter physics}

\subsection{Introduction}

Neutron scattering is a collective name for the technique of measuring atomic spacing by using neutrons as a probe. Neutrons like other particles exhibit wave properties. Thermal neutrons and cold neutrons have wavelengths ranging from 1 to $30 \AA$ (Table 5.1.1) [17]. The atomic spacing in condensed matter is known to be around this range. According to Bragg's law, the atomic spacing, $d$, can be determined by the constructive interference when the path difference of two beams of particles with the same wavelength equals some integer multiple of its wavelength (Figure 5.1.1).

$$
n \lambda=2 d \sin \theta
$$

where $\quad \lambda$ is the wavelength of incident neutron, and

$\theta$ is an angle that the incident beam makes with one of the planes of atoms. 


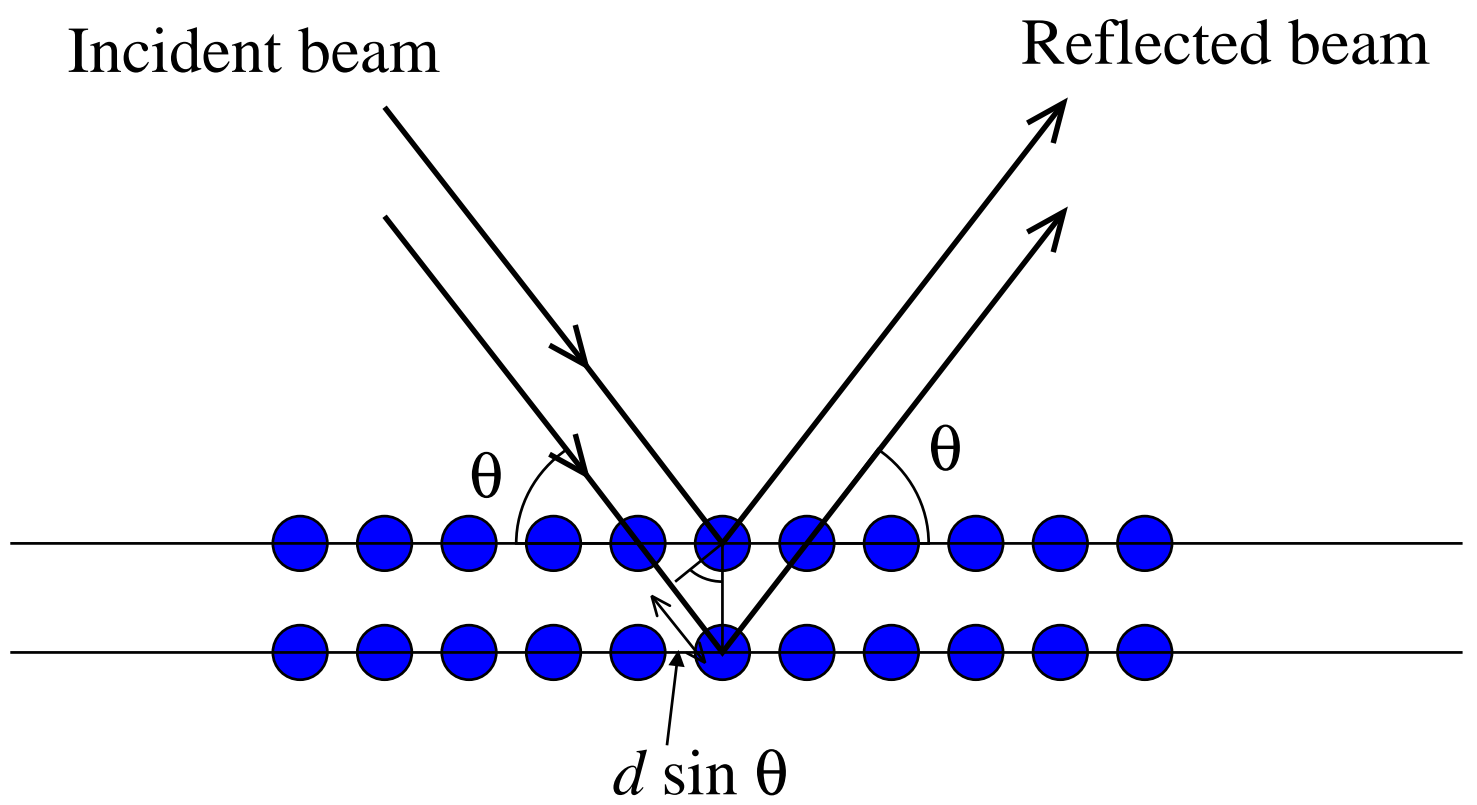

Figure 5.1.1 A two-dimensional representation of Bragg's law

The regular array of atoms in a crystal could act as a three-dimensional diffraction grating for any particle exhibiting wave properties.

\begin{tabular}{|l|l|l|l|l|}
\hline \multicolumn{1}{|c|}{ Source } & \multicolumn{1}{c|}{ Energy $(\mathrm{meV})$} & $\begin{array}{c}\text { Temperature } \\
\left({ }^{\circ} \mathrm{K}\right)\end{array}$ & Wavelength $(\AA)$ & Velocity $(\mathrm{m} / \mathrm{s})$ \\
\hline cold & $0.1-10$ & $1-120$ & $30-3$ & $170-1700$ \\
\hline thermal & $5-100$ & $60-1200$ & $4-1$ & $1200-5400$ \\
\hline hot & $100-500$ & $1200-5800$ & $1-0.4$ & $5400-12000$ \\
\hline
\end{tabular}

Table 5.1.1 Properties of neutrons for neutron scattering experiments

Besides that thermal and cold neutrons have wavelength closed to the atomic spacing of condensed matter, neutrons have an advantage of being neutral. Unlike X-rays and electrons, neutrons interact with the nucleus rather than its electron cloud. Thus, neutrons are highly penetrating and sensitive to light atoms. The reaction cross section of neutrons varies with different isotopes. This property also allows scientists to exploit 
techniques such as isotopic substitution and contrast variation to differentiate complex molecular structures.

\subsection{Thermal neutron beam for diffraction experiments}

The goal of this design is to produce a thermal neutron beam that is suitable for thermal neutron scattering experiments. Unlike spallation neutron sources, the pulse width of a fusion-based neutron generator is usually larger because of the duty factor. The duty factor is defined as the product of ion beam pulse width multiplied by the operating frequency. If the pulse width is too short, the beam current during the pulse needs to be increased in order to produce the same time average neutron yield. The current is limited by the plasma density in the source. The operating frequency is

determined by the distance of flight path and the neutron energies. A reasonable length of flight path must be assumed to avoid noise signals from the previous pulse. The pulse width is assumed to be $50 \mu$ s and the operating frequency is assumed to be $80 \mathrm{~Hz}$. This gives a duty factor of $0.4 \%$.

\subsubsection{Design parameters for thermal neutron scattering system and error analysis}

It is important to identify the parameters that determine the resolution of a neutron scattering system. The simulation is very different between a thermal neutron scattering system and a cold neutron scattering system. One main difference is that the neutron guides are used in cold neutron scattering. MCNP does not have the capability of simulating the effects of a neutron guide. Therefore, the simulation of thermal neutron scattering is discussed here first. When TOF technique is used, Bragg's Law can be re-written as the following: 


$$
\begin{aligned}
& \lambda=\frac{h t}{m L} \\
& d=\frac{n h t}{2 m L \sin \theta}
\end{aligned}
$$

where $L$ is the total length of the flight path from moderator to sample to detector, $t$ is the time of flight for the neutron.

The resolution is defined as:

$$
R=\frac{\delta_{d}}{d}
$$

where $\quad \delta_{d}$ is the FWHM at $d$.

Unfortunately, MCNP does not take lattice orientation into account in its calculation so it is impossible to calculate the exact resolution of the whole system. There is specific software for neutron instrument designs. However, these codes do not solve the general problems that MCNP can deal with. As a result, some assumption had been made in the MCNP calculation to obtain an upper bound of $R$.

The first assumption is that $L$ is constant. The flight path, $L$, has small variation due to the incident angle of neutron, the sample size, and the angle covered by one single detector tube being seen by a neutron. A typical maximum sample size for High Pressure Preferred Orientation (HIPPO) diffractometer is $2 \mathrm{~cm}$ in diameter.[18] The incident angle is determined by the collimator of the system. In the calculation for thermal neutron scattering system described in this thesis, the incident neutron beam divergence is 0.32 degree. A detector bank has a plane geometry and is usually tilted with an angle so the exact length of the flight path from sample to detector is unknown. This tilted angle depends on the length of the detector tube and the scattered angle, 2 $\theta$. The two factors 
change the sample to detector distance, $L_{2}$. The deviation of the flight time due to sample size and the angular divergence of the neutron beam can be taken into account by simulating the problem with the exact geometry in MCNP. However, $L$ cannot be measured for each neutron. Fortunately, the deviation of $L$ is comparatively small (less than $1 \%)$.

Because $R$ will be under-estimated by assuming a constant $L$, another assumption is made to avoid underestimating $R$. The second assumption is that $R$ is calculated at the sample position, $L_{1}$. Because $L_{1}$ is shorter than $L$ (i.e. $L_{1}+L_{2}$ ), the resolution calculated at sample position can be used as an upper bound for the system regardless of $L_{2}$.

The third assumption is that there is no angular divergence in the beam when a range of neutron energies is chosen for each detector tube in the calculation for a specific d. Because MCNP does not take lattice orientation into account, the angular divergence must be converted into an energy range for the calculation of $\delta_{d}$. As shown in Figure 5.2.1, the angular divergence becomes negligible when the angle covered by the detector panel is large. Although the energy range is calculated from a beam with no angular divergence, MCNP will take the angular divergence of the beam within the calculated energy range into account. The detector tubes in Figure 5.2.1 are aligned into the page. (NOTES: Figure 5.2.1 is not to scale. The moderator to sample distance, $L_{1}$, is 3 to 5 times larger than the sample to detector distance, $L_{2}$, depending on the scattered angle, $2 \theta$. The angular divergence of the beam due to collimation is also much smaller than the scattered angle.) 


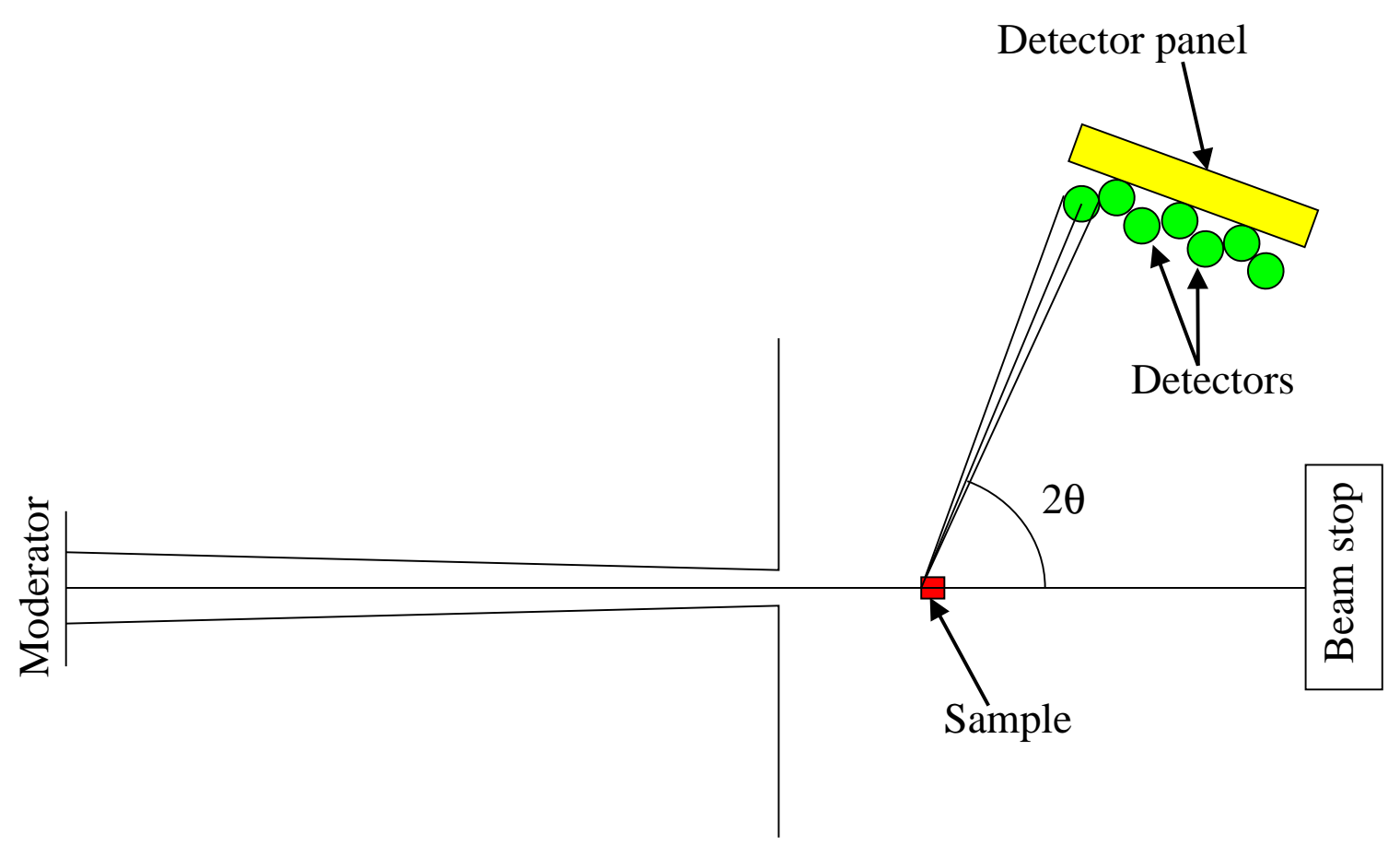

Figure 5.2.1 A schematic diagram showing the relationship between the detector panel and sample position

The assumptions made in the calculation can be examined by the following error analysis. The error of $d$ due to the error propagation of $\theta$ and $t$ derived from Bragg's Law can be written as:

$$
\left(\frac{\delta_{d}}{d}\right)^{2}=\left(\frac{\delta_{t}}{t}\right)^{2}+\left(\frac{\delta_{\theta}}{\theta}\right)^{2}
$$

where $\quad \delta_{t}$ is the uncertainty due to $t$.

$\delta_{\theta}$ is the uncertainty due to beam divergence.

There are several uncertainties neglected in the Equation 5.5 because of the assumptions mentioned above. A complete version of this equation is derived with consideration of uncertainties due to $L$, size of the sample and size of detector [19] : 


$$
\left(\frac{\delta_{d}}{d}\right)^{2}=\left(\frac{\delta_{t}}{t}\right)^{2}+\left(\frac{\delta_{L}}{L}\right)^{2}+\left[\left(\frac{W_{d e t}}{L_{2}}\right)^{2}+\left(\frac{W_{\text {sample }}}{L_{1}}\right)^{2}+\delta_{\theta}^{2}\right] \cdot \cot ^{2}
$$

where $\quad W_{d e t}$ is the diameter of the detector tube.

$W_{\text {sample }}$ is the width of the sample.

The uncertainty due to variation of $L$ can be taken into account by $t$ if exact geometry can be modeled in the simulation. As mentioned previously, it will be too tedious to calculate the exact $L$ in MCNP for each neutron. It is assumed to be $L_{1}$ and its uncertainty is included in $\delta_{\mathrm{t}} . W_{\text {sample }} / L_{1}$ is approximately equal to the angular divergence of the neutron beam. Because the geometry is treated exactly in the simulation up to the sample position, the uncertainty due to this angular divergence is also taken into account by $t . W_{d e t} / L_{2}$ is approximately equal to the angular divergence due to diameter of one detector tube. A typically diameter for a detector tube is approximately $1.25 \mathrm{~cm}$. The flight path from sample to detector range from $0.75 \mathrm{~m}$ to $2 \mathrm{~m}$. Therefore, the angle is much smaller than the scattered angle. The incident beam divergence, $\delta_{\theta}$, is converted into energy range and taken into account. Therefore, the angular divergence due to the detector size is partially included in our calculation. Our overall assumptions will slightly overestimate $R$ because the actual length of flight path will reduce uncertainty more than those uncertainties that had been ignored.

\subsubsection{Computational model for a thermal neutron scattering facility}

A model of the system was created with the geometry shown in Figure 5.2.2. A conical D-T neutron generator is used for the thermal neutron scattering facility. The neutron generator consists of two conical shape targets with a base diameter of $8 \mathrm{~cm}$ and 
a height of $8 \mathrm{~cm}$. The aluminum backing for the neutron generator is assumed to be 0.45 $\mathrm{cm}$ thick. Ti on the $\mathrm{Al}$ backing is not specified in the input file because the $\mathrm{Ti}$ is so thin that it has very little effects in terms of neutron moderation. The conical neutron generator is surrounded by a Be moderator which has a rectangular shape. The rectangular Be moderator has a dimension of $32 \mathrm{~cm}$ by $32 \mathrm{~cm}$ and $16 \mathrm{~cm}$ thick. A polyethylene moderator next to the Be moderator with a surface area of $13 \mathrm{~cm}$ by $13 \mathrm{~cm}$ and $4 \mathrm{~cm}$ thick. The polyethylene moderator is surrounded by a Ni reflector.

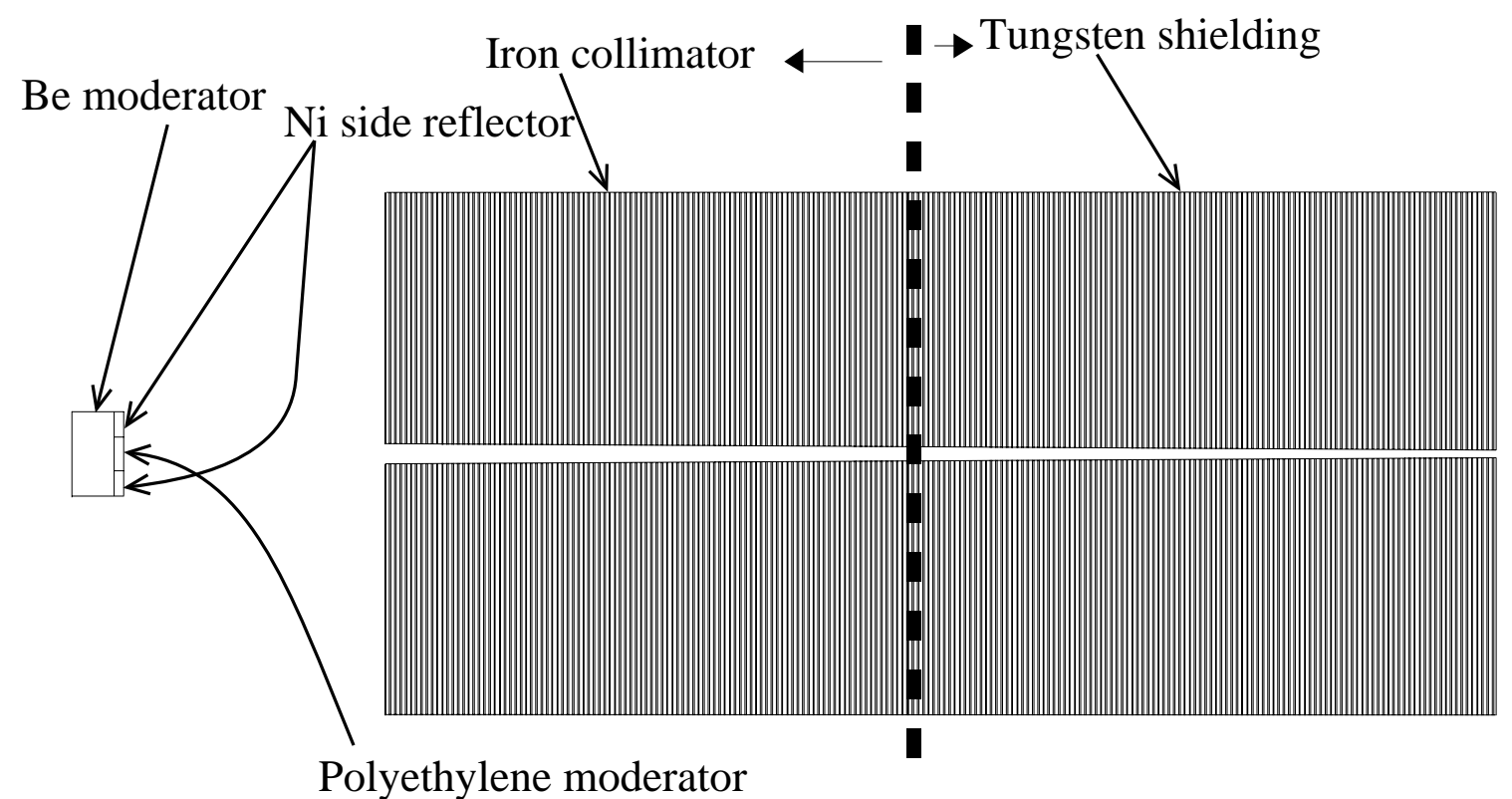

Figure 5.2.2 Geometry of thermal neutron scattering model in MCNPX

The collimator system is $1 \mathrm{~m}$ away from the polyethylene moderator surface. It views a circular portion of the moderator surface $8.7 \mathrm{~cm}$ in diameter so that the thermal neutron beam divergence is $0.32^{\circ}$. The collimation system is very similar to the collimation system for HIPPO diffractometer at LANSCE in order to make the results comparable to a spallation neutron source.[18] The first two meters of the collimator system consists of $1.5 \mathrm{~cm}$ thick iron plates. Each iron plate is separated by $0.5 \mathrm{~cm}$. The 
iron collimator system is followed by a $2.25 \mathrm{~m}$ tungsten collimator system, which is made up of tungsten plates with the same thickness and spacing between as the iron collimator system. The sample is 6 meter away from the surface of polyethylene moderator and has a diameter of $2 \mathrm{~cm}$.

Table 5.2.1 shows four detector banks of the HIPPO diffractometer and its angular range in each bank.[18] Each detector bank is capable of detecting scattered neutrons within a certain range of energy. A list of atomic spacing, $d$, (the $4^{\text {th }}$ column on the table) is chosen for the calculation. For short pulse spallation neutron source (SPSS), the primary proton beam has a width of $560 \mathrm{~ns}$, which is small compared to the time required for neutron moderation. For fusion-based neutron generators, a relatively long ion pulse width $(50 \mu \mathrm{s})$ is used due to the consideration of the low duty factor and the large heat flux on the target during the pulse. The effects of source neutron pulse width are shown in the next section. The flight path of HIPPO diffractometer at LANSCE is 3 $\mathrm{m}$ longer than the flight path of this fusion-based neutron scattering system. A short flight path can improve the duty factor as the source can operate at a higher frequency. The trade off of a higher pulsing frequency is energy resolution when hot or thermal neutrons are considered. Most calculations are performed at $20^{\circ}$ because the computer time increases with the number of neutron tallies. For the detector bank at $20^{\circ}$, six $d$ 's are chosen so there are six energy bins in each tally. Each detector tube on a detector bank gives a slight different angle. Because the detector bank at $20^{\circ}$ has 32 detector tubes, 32 neutron tallies are used. For each energy bin, there are 50 time bins in order to get a neutron pulse shape as a result. Therefore, 32 matrices with 2555 elements per matrix need to be allocated for the calculation of $d$ at $20^{\circ}$. The computer time will significantly 


\begin{tabular}{|c|c|c|c|c|c|}
\hline $\begin{array}{l}\text { Nominal } \\
\text { Angle }\end{array}$ & Angular Range & $\mathrm{L}_{2}(\mathrm{~m})$ & d $(\AA)$ & $\begin{array}{l}\text { Energy } \\
(\mathrm{meV})\end{array}$ & Energy Range (meV) \\
\hline \multirow[t]{7}{*}{150} & $143^{\circ} 54^{\prime}-155^{\circ} 48$ & 1.25 & 0.36 & 166 & $162-172$ \\
\hline & & & 0.52 & 80 & $77.7-82.1$ \\
\hline & & & 0.76 & 38 & $37.1-39.3$ \\
\hline & & & 1.1 & 18.2 & $17.8-18.8$ \\
\hline & & & 1.6 & 8.7 & $8.5-9.0$ \\
\hline & & & 2.3 & 4.2 & $4.1-4.3$ \\
\hline & & & 3.3 & 2 & $1.94-2.05$ \\
\hline \multirow[t]{7}{*}{90} & $76^{\circ} 46^{\prime}-103{ }^{\circ} 15^{\prime}$ & 0.75 & 0.52 & 153 & $125-199$ \\
\hline & & & 0.75 & 73 & $59-95$ \\
\hline & & & 1.1 & 35 & $28-45$ \\
\hline & & & 1.6 & 16.6 & $13.5-21.6$ \\
\hline & & & 2.3 & 7.9 & $6.4-10.3$ \\
\hline & & & 3.3 & 3.8 & $3.1-4.9$ \\
\hline & & & 4.8 & 1.8 & $1.5-2.3$ \\
\hline \multirow[t]{7}{*}{40} & $33^{\circ} 29^{\prime}-47^{\circ} 35^{\prime}$ & 1 & 1.06 & 157 & $113-221$ \\
\hline & & & 1.5 & 75 & $54-106$ \\
\hline & & & 2.2 & 36 & $25.8-50.7$ \\
\hline & & & 3.2 & 17.2 & $12.4-24.3$ \\
\hline & & & 4.6 & 8.2 & $5.9-11.6$ \\
\hline & & & 6.7 & 3.9 & $2.8-5.6$ \\
\hline & & & 9.6 & 1.9 & $1.4-2.7$ \\
\hline \multirow[t]{6}{*}{20} & $18^{\circ} 17^{\prime}-25^{\circ} 47^{\prime}$ & 1.5 & 1.97 & 175 & $106-209$ \\
\hline & & & 2.85 & 83.7 & $50.7-100$ \\
\hline & & & 4.12 & 40 & $24.2-47.8$ \\
\hline & & & 8.62 & 9.1 & $5.5-10.9$ \\
\hline & & & 12.5 & 4.36 & $2.6-5.2$ \\
\hline & & & 18.04 & 2.1 & $1.26-2.49$ \\
\hline
\end{tabular}

Table 5.2.1 Range of energy for different atomic spacing and angle ${ }^{1}$

1 There is a $10^{\circ}$ detector bank for HIPPO diffractometer not listed in this table. 
increase if two detector banks are calculated in one problem. The $20^{\circ}$ detector bank was chosen for most calculation because resolution gets better with larger angles. A DXTRAN sphere with a diameter of $2 \mathrm{~cm}$ is used at the sample position to bias the scattered direction to the sample position in order to reduce the variance of the results.

\subsubsection{Computational results for a thermal neutron scattering facility}

The neutron tallies with time bins are obtained at the sample position with MCNPX. The time is converted to atomic spacing, $d$. The detector tube with largest scattered angle in one detector bank is taken as a reference for calibrating $d$. The average of maximum and minimum neutron energy seen by this detector tube is used to calculate the velocity of neutron. By assuming the flight path is fixed, $d$ is proportional to the flight time. Therefore, $d$ calculated from each time bin is scaled by this reference $d$ and flight time. By shifting the time bins with the same size of $d$, the neutron tallies in other energy bins (i.e. other detector tubes on the same detector bank) can be converted to $d$ 's. The total thermal and cold neutron flux at the sample position is $7.3 \times 10^{5} \mathrm{n} / \mathrm{cm}^{2} \mathrm{~s}$. The resolutions for the detector bank at $20^{\circ}$ is $<5 \%$. The results show that the cold neutrons have a better resolution than the thermal and hot neutrons. The resolution of the neutron scattering system depends on the angular resolution, the length of flight path and ion beam pulse width. The ion beam pulse width is chosen to be $50 \mu \mathrm{s}$. The flight path is assumed to be $6 \mathrm{~m}$ (i.e. the moderator to sample distance) for the reasons discussed in the previous section. The angular resolution is a function of the neutron beam divergence, the detector tube diameter and the sample to detector distance, $L_{2}$. At the $20^{\circ}$ detector bank, the resolution for $d>8 \AA$ is $<2 \%$ (See Figure 5.2.3 to Figure 5.2.5). These figures show 
$18.04 \mathrm{~A}$ at $2 \theta=20$

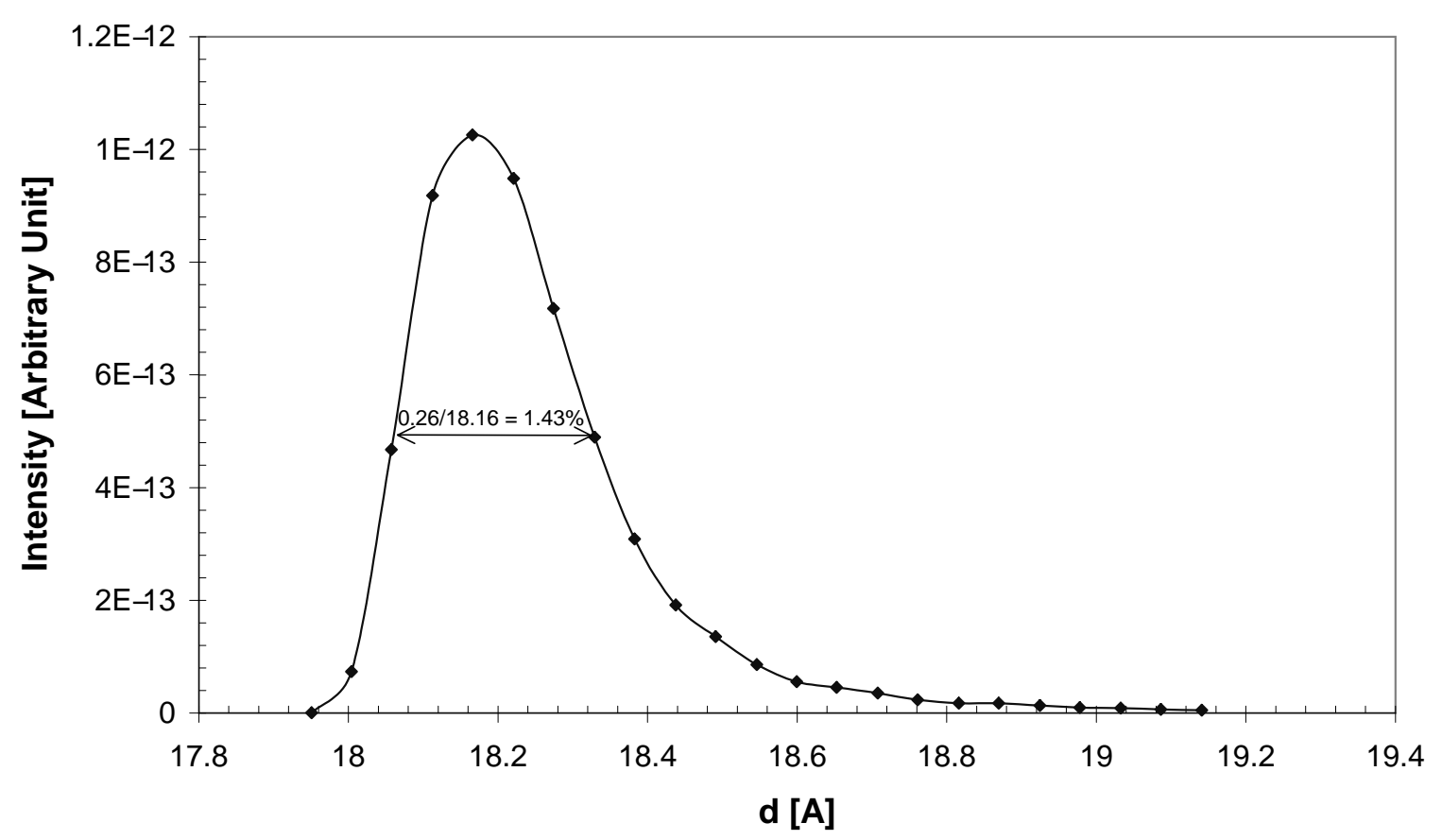

Figure 5.2.3 Resolution at $d=18.04 \AA$ at $2 \theta=20^{\circ}$ (ion beam width $=50 \mu \mathrm{s}$ )

\section{$12.5 \mathrm{~A}$ at $2 \theta=20 \mathrm{deg}$}

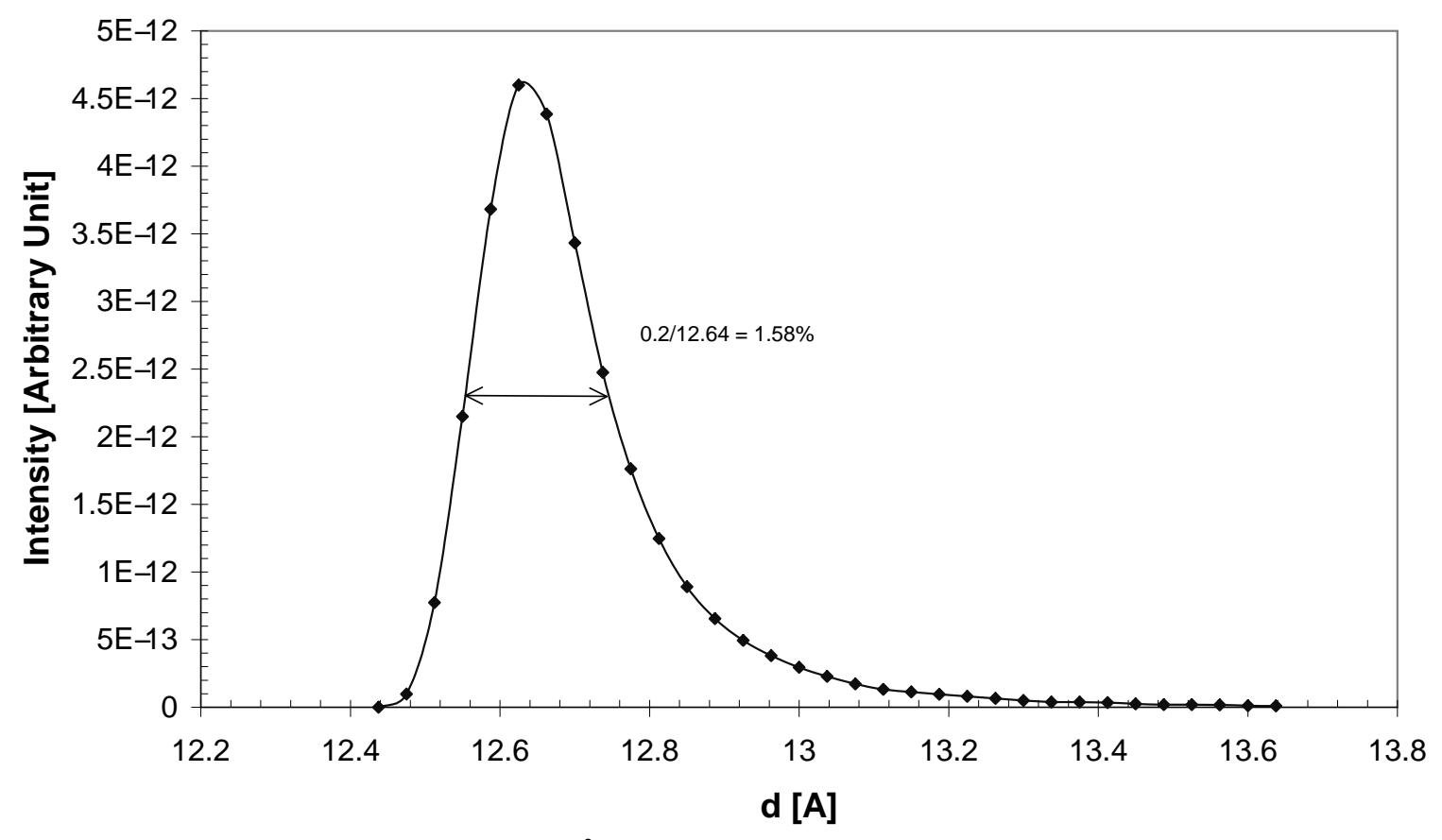

Figure 5.2.4 Resolution at $d=12.5 \AA$ at $2 \theta=20^{\circ}$ (ion beam width $=50 \mu \mathrm{s}$ ) 
that the cold neutrons have an excellent resolution for the fusion-based neutron scattering system because the flight time of cold neutrons is long. The thermal and hot neutrons have a short flight time.

\subsection{A at $2 \theta=20 \mathrm{deg}$}

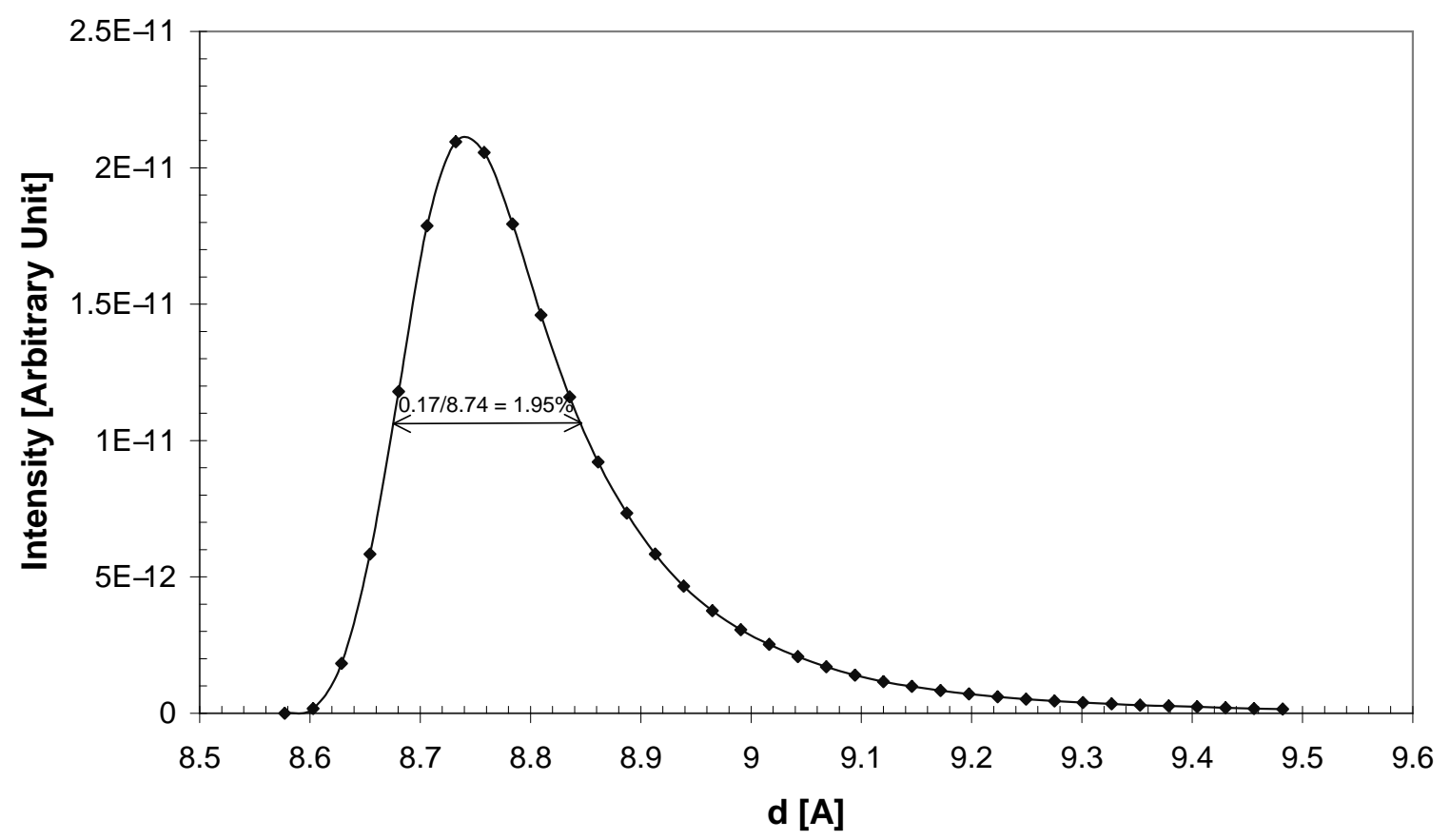

Figure 5.2.5 Resolution at $d=8.62 \AA$ at $2 \theta=20^{\circ}$ (ion beam width $=50 \mu \mathrm{s}$ )

The cold neutrons of this system have better resolution than the thermal or hot neutrons due to the intrinsic long moderation time and flight time of cold neutron. The pulse width of thermal neutrons differs from that of cold neutrons. The pulse width is a function of ion beam pulse width and the moderation time. Cold neutrons have a long moderation time. The long moderation time spreads the initial neutron pulse so much that the signals from one detector tube overlaps with the signals from the adjacent detector tubes in a certain number of time frames. The moderation time of cold neutrons is also comparable to the ion beam pulse width. On the contrary, the pulse width of thermal or 
hot neutrons does not spread too much and does not overlap. Therefore, the ion beam pulse width has a significant effect on the $\delta_{\mathrm{t}}$ of thermal and hot neutrons. Figure 5.2.6 and Figure 5.2.7 show the effects of ion beam pulse width on the resolution of hot neutrons.

\section{$1.97 \mathrm{~A}$ at $2 \theta=20 \mathrm{deg}$}

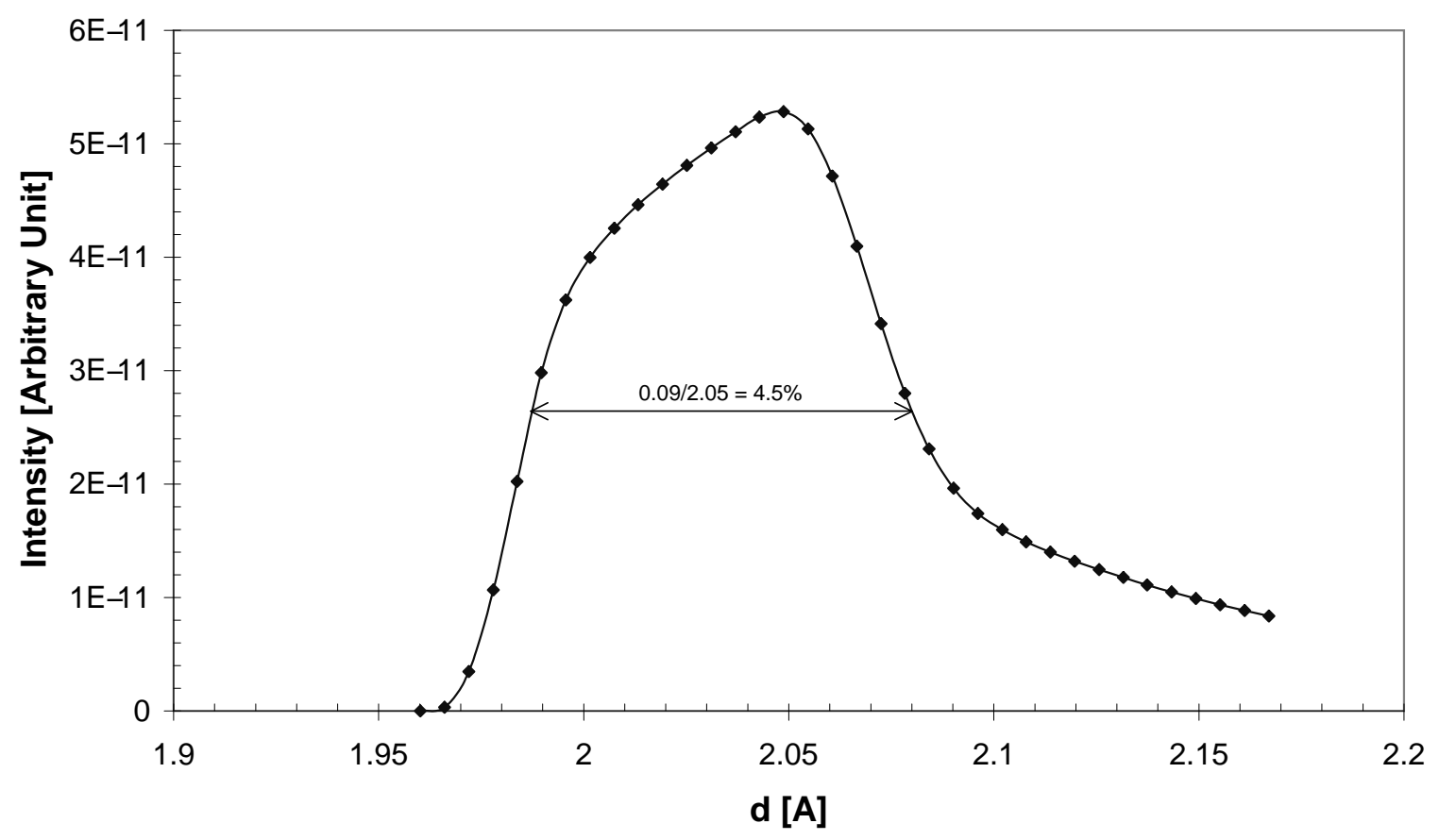

Figure 5.2.6 Resolution at $d=1.97 \AA$ at $2 \theta=20^{\circ}$ (ion beam width $=50 \mu \mathrm{s}$ )

The resolution of $d=1.97 \AA$ at $20^{\circ}$ is $4.5 \%$ when the pulse width of ion beam is $50 \mu$ s (Figure 5.2.6). The resolution of the same $d$ at the same angle is $1.26 \%$ when the width of source neutron emission time (i.e. ion beam pulse width) is zero (Figure 5.2.7). Notice that the hot neutrons have a long tail. This tail can be reduced by placing a neutron absorber a few centimeters from the surface of moderator. However, using a neutron absorber reduces the neutron flux. Figure 5.2.8 shows that the resolution of $d=2.85 \AA$ at $20^{\circ}$ is $4.4 \%$. 


\section{$1.97 \mathrm{~A}$ at $2 \theta=20 \mathrm{deg}$}

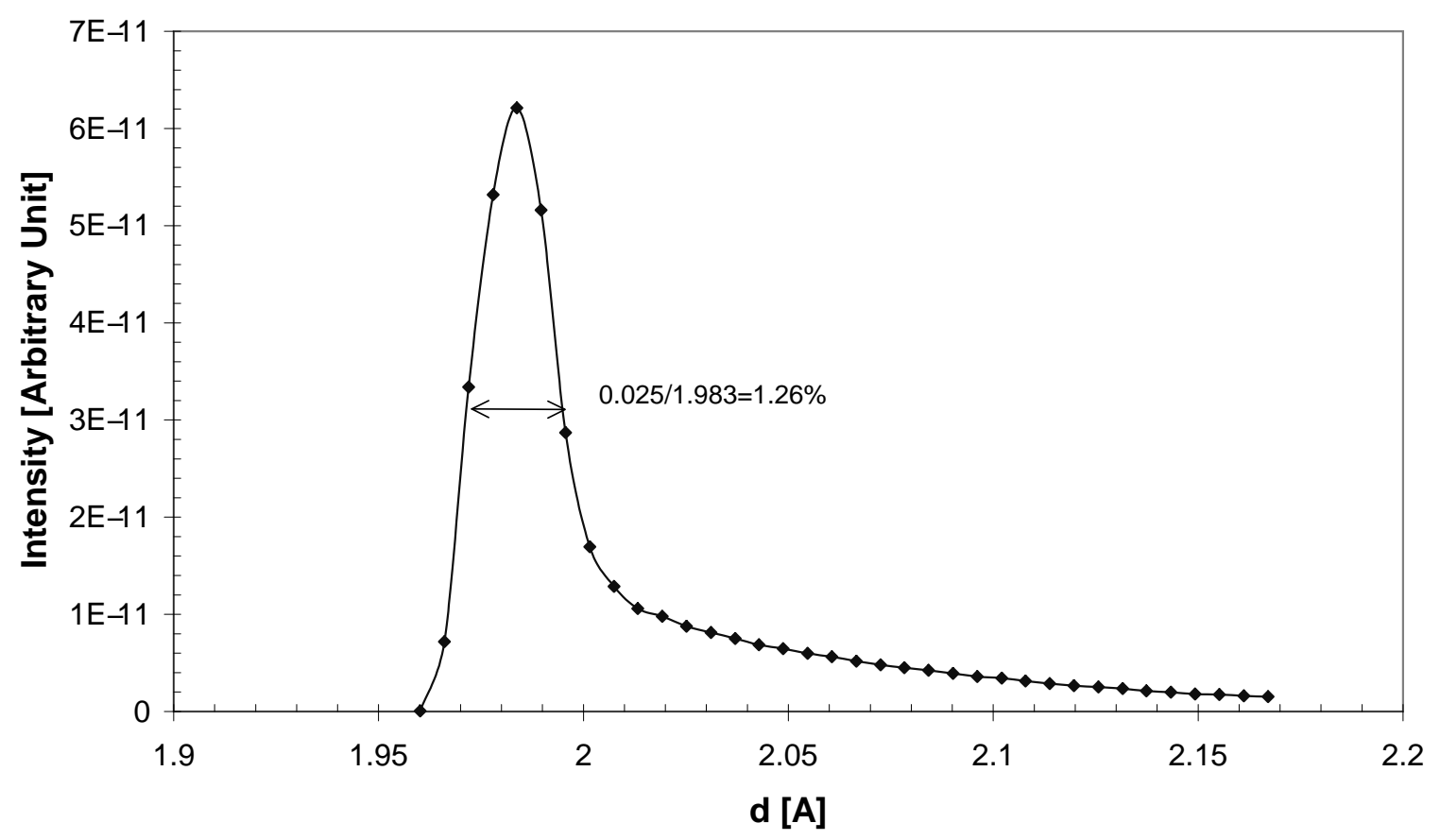

Figure 5.2.7 Resolution at $d=1.97 \AA$ at $2 \theta=20^{\circ}$ (ion beam width $=0 \mathrm{~s}$ )

\section{$2.85 \mathrm{~A}$ at $2 \theta=20 \mathrm{deg}$}

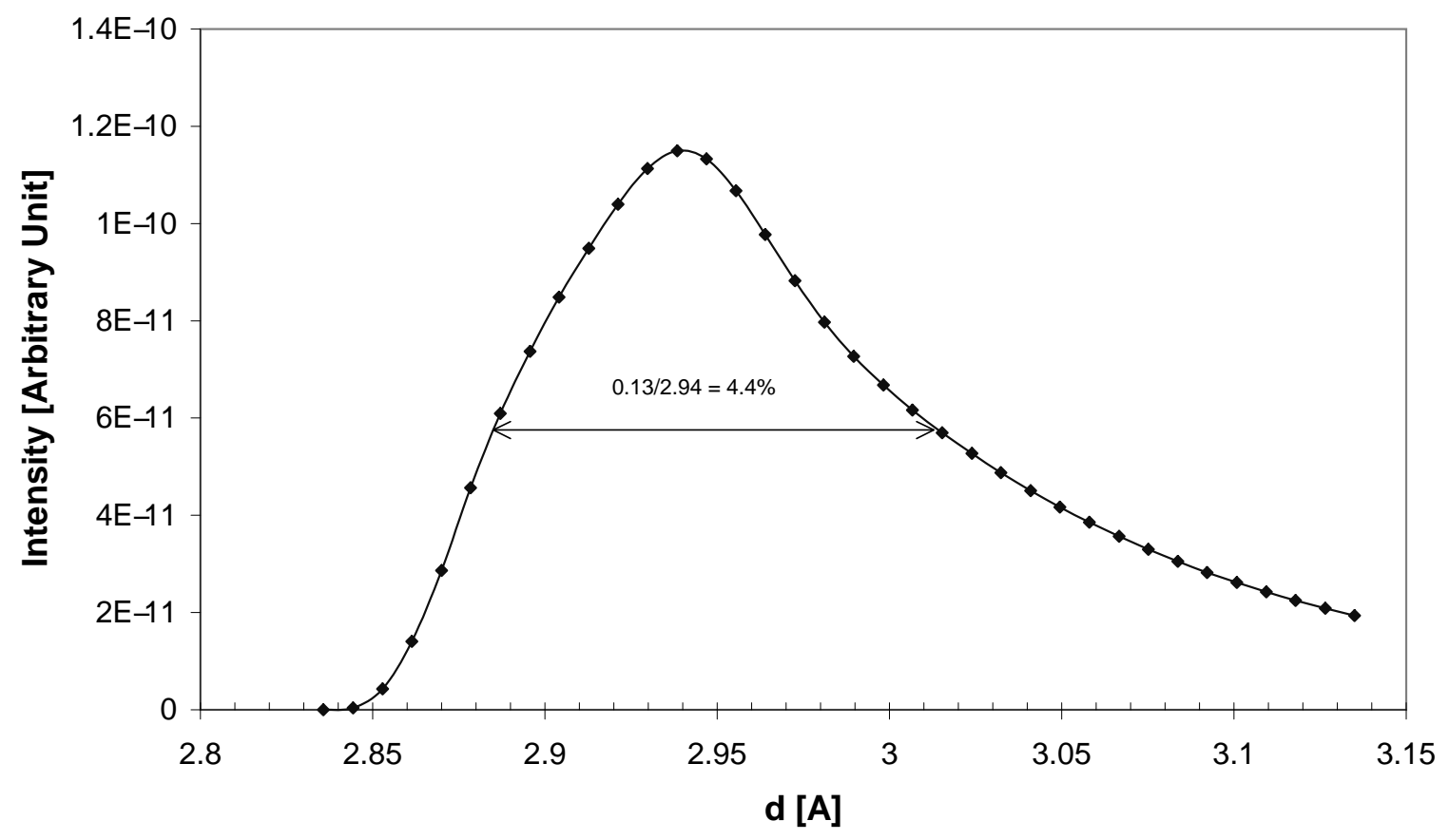

Figure 5.2.8 Resolution at $d=2.85 \AA$ at $2 \theta=20^{\circ}$ (ion beam width $=50 \mu \mathrm{s}$ ) 
The energy range covered by each detector tube decreases as the scattered angle, $2 \theta$, increases. The measurable atomic spacing also dereases as the scattered angle increases. Therefore, the resolution does not improve with larger scattered angle for thermal neutrons with the same energy because both $\delta_{\mathrm{d}}$ and $d$ decrease at about the same rate.

Figure 5.2.9 shows that the resolution at $20^{\circ}$ for $d=4.12 \AA$ is $3.55 \%$. The energy range covered by this detector bank is $24.2 \mathrm{meV}$ to $47.8 \mathrm{meV}$. The resolution at $150^{\circ}$ for $d=0.76 \AA$ is $3.5 \%$ as shown in Figure 5.2.10. The energy range covered by this detector bank is $37.1 \mathrm{meV}$ to $39.3 \mathrm{meV}$. The small difference in the resolutions of these two cases confirms that the scattered angle has very little effects on the resolution of thermal and

\subsection{2 $\mathrm{A}$ at $2 \theta=20 \mathrm{deg}$}

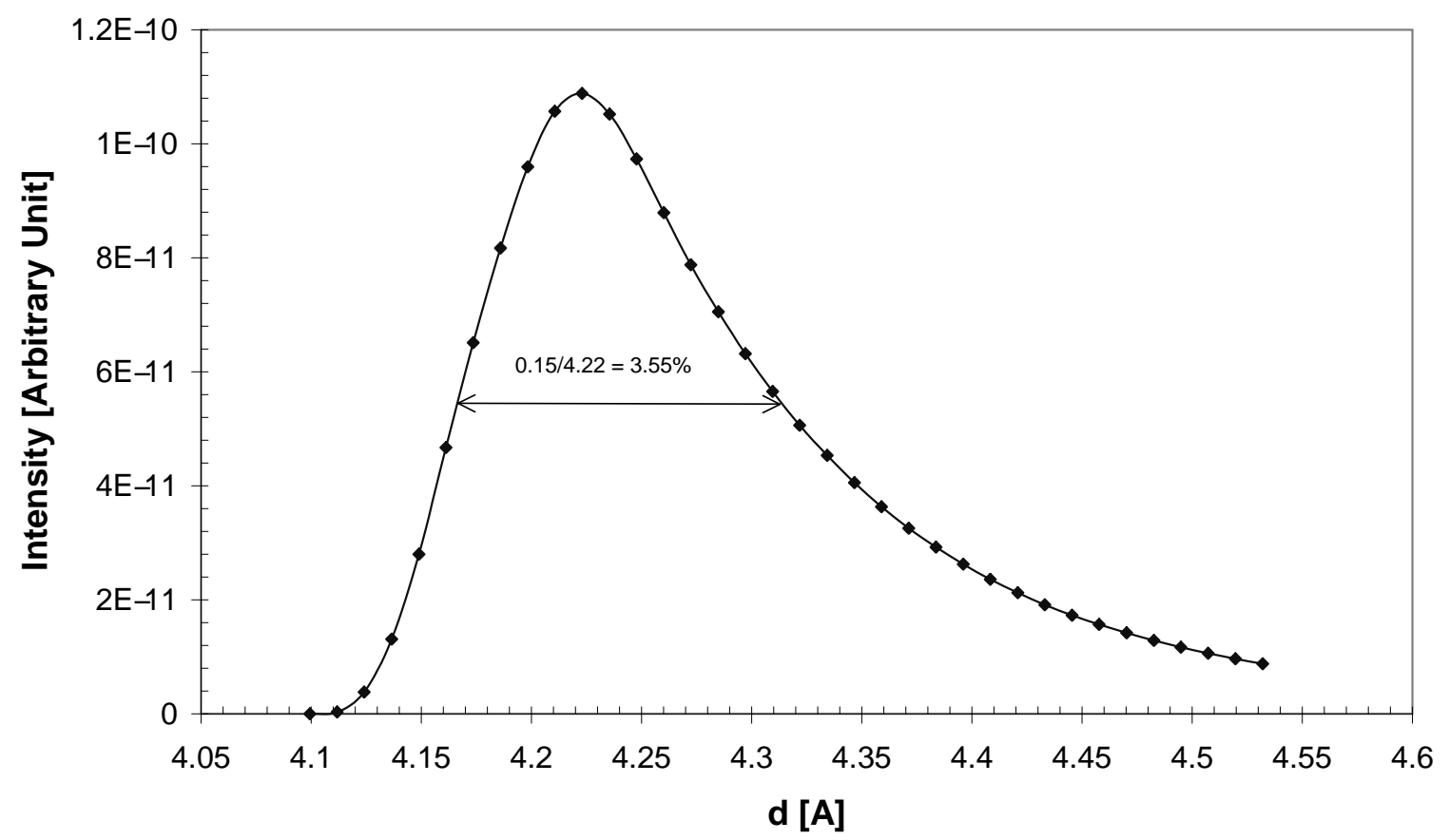

Figure 5.2.9 Resolution at $d=4.12 \AA$ at $2 \theta=20^{\circ}$ (ion beam width $=50 \mu \mathrm{s}$ ) 
hot neutrons. For cold neutrons, the resolution improves slightly because the pulse width for cold neutrons is larger.

\section{$0.76 \mathrm{~A}$ at $2 \theta=150 \mathrm{deg}$}

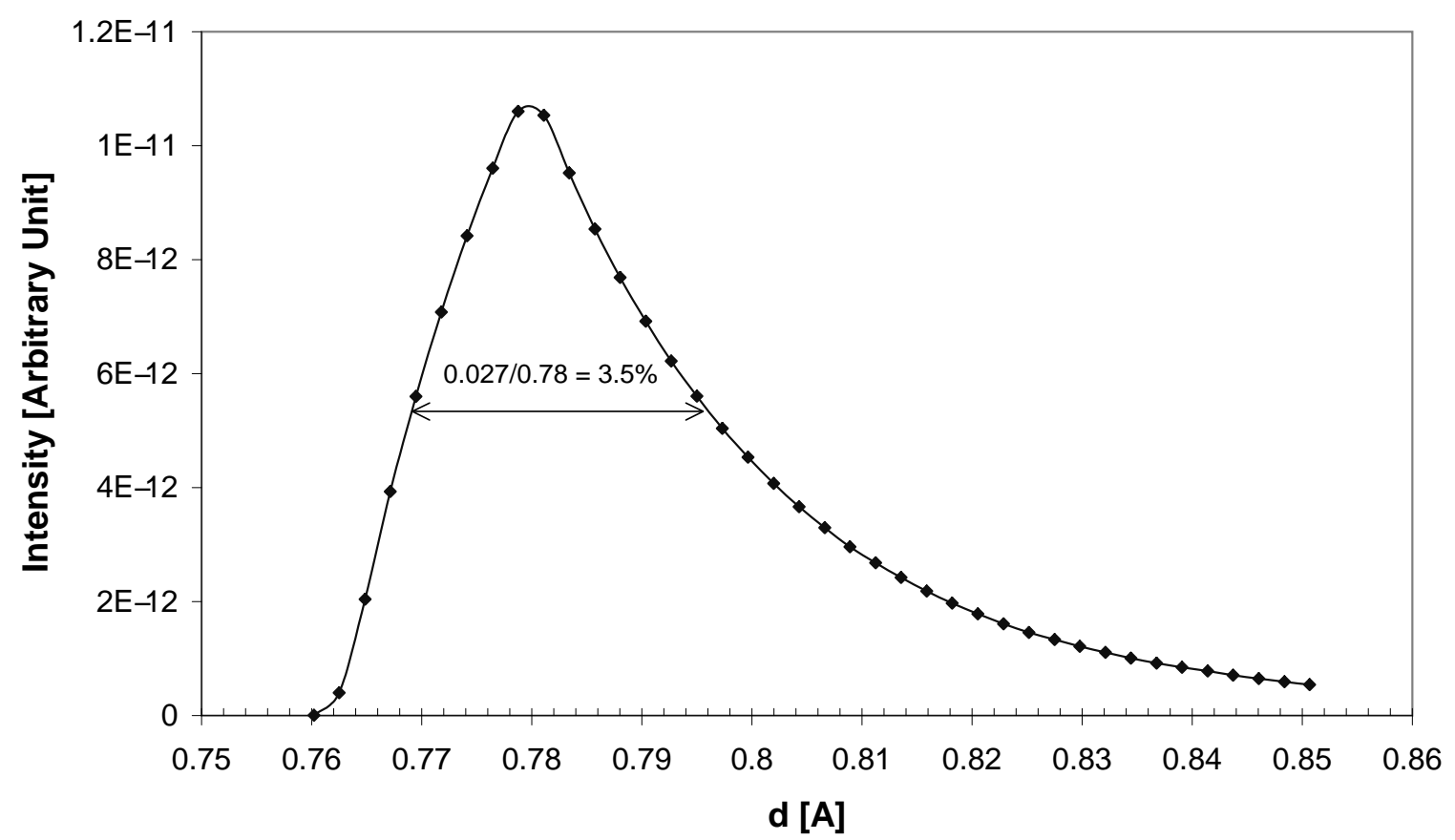

Figure 5.2.10 Resolution at $d=0.76 \AA$ at $2 \theta=150^{\circ}$ (ion beam width $=50 \mu \mathrm{s}$ )

The resolution at $150^{\circ}$ for $d=3.3 \AA$ is $1.2 \%$ as shown in Figure 5.2.11. The energy range covered by this detector bank is $1.94 \mathrm{meV}$ to $2.05 \mathrm{meV}$. This resolutions is better than the resolution of $1.43 \%$ at $20^{\circ}$ for $d=18.04 \AA$. The energy range covered by this detector bank is $1.26 \mathrm{meV}$ to $2.49 \mathrm{meV}$. The resolution of cold neutrons is improved by 15 to $20 \%$ when the scattered angle is changed from $20^{\circ}$ to $150^{\circ}$ while the resolution for hot neutrons is improved by $<2 \%$.

The resolution of HIPPO diffractometer at LANSCE is $2.6 \%$ at $20^{\circ}$ and $5.0 \%$ at $10^{\circ}$. Because the detector banks at larger angle can be used for larger $d$ if better resolutions are needed, a resolution of $<5 \%$ for a fusion-based thermal neutron scattering 


\section{$3.3 \mathrm{~A}$ at $2 \theta=150$}

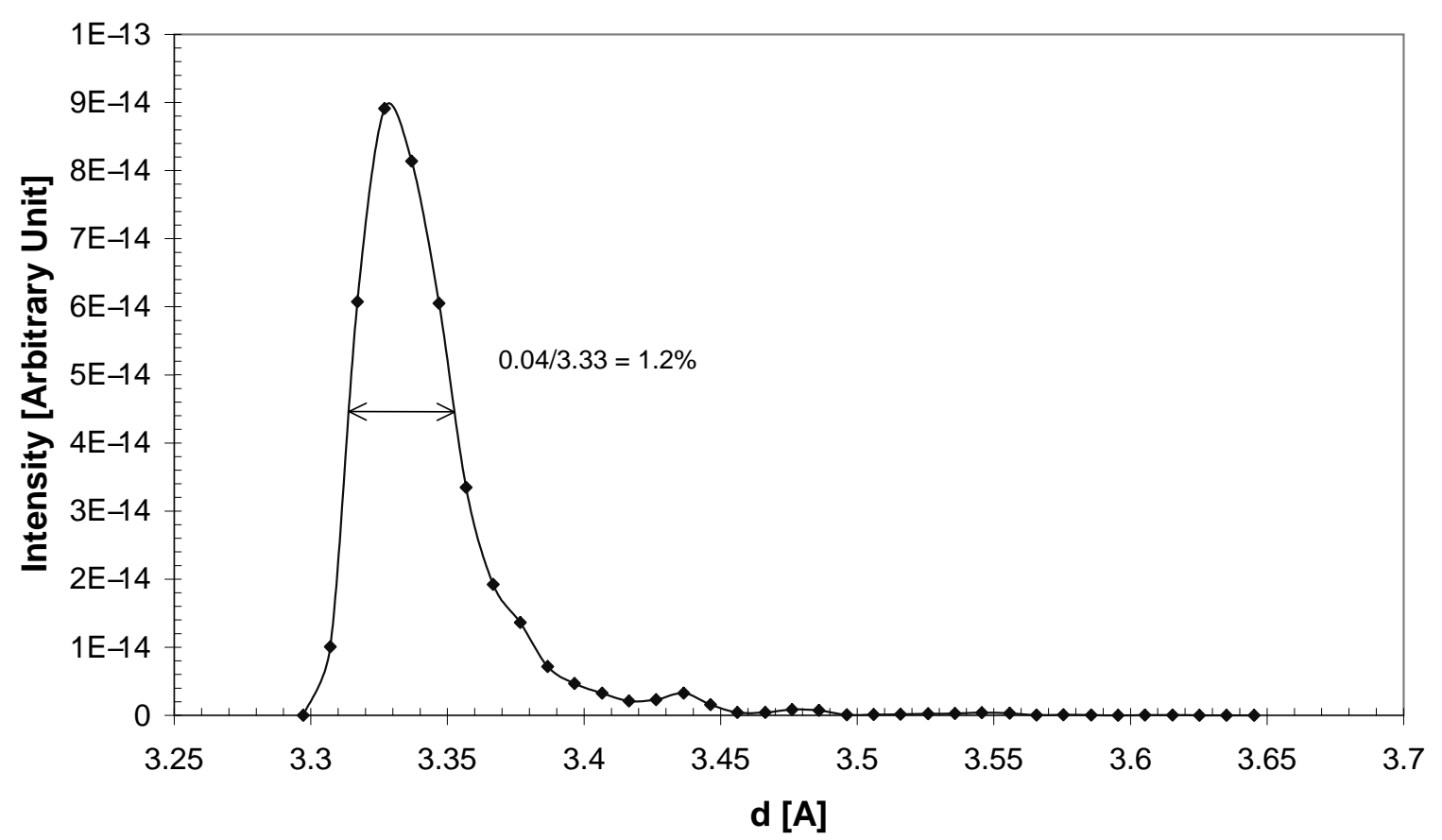

Figure 5.2.11 Resolution at $d=3.3 \AA$ at $2 \theta=150^{\circ}$ (ion beam width $=50 \mu \mathrm{s}$ )

system can be obtained. The results show that the error is mainly due to $\delta_{l} / t$ for hot and thermal neutrons and due to $\delta_{\theta} / \theta$ as expected. This observation matches the error analysis presented in the previous section.

\subsection{Cold neutron source for Small Angle Neutron Scattering}

As shown in the results for the thermal neutron scattering facility, the fusionbased neutron generator have a greater potential to be a cold neutron source for Small Angle Neutron Scattering (SANS) because of the better resolution of cold neutrons when the ion beam pulse width is large $(\sim 50 \mu \mathrm{s})$. The cryogenic moderator for a cold neutron source can be surrounded with a large beryllium and graphite reflectors. In this case, a neutron source of $10^{14} \mathrm{n} / \mathrm{s}$ can produce a peak moderator brightness of $6 \times 10^{12} \mathrm{n} / \mathrm{cm}^{2}$-s-sr- 
$\mathrm{eV}$. The moderator brightness is defined as the neutron flux on the moderator surface (i.e. number of neutrons per unit moderator area per unit time) per steradian per unit energy. Figure 5.3.1 shows one neutron brightness curve obtained from a $10^{14} \mathrm{n} / \mathrm{s}$ D-T neutron source. This D-T neutron source has a $2 \mathrm{~cm}$ thick and $10 \mathrm{~cm}$ diameter cryogenic liquid Para-hydrogen moderator. Before the source neutrons reach the cryogenic moderator, they go through a $3.5 \mathrm{~cm}$ thick beryllium moderator. The neutron generator and moderators are enclosed by a beryllium reflector. The beryllium reflector has a dimension of $1 \mathrm{~m}$ height and $1 \mathrm{~m}$ diameter. The design is not optimized and its neutron emission

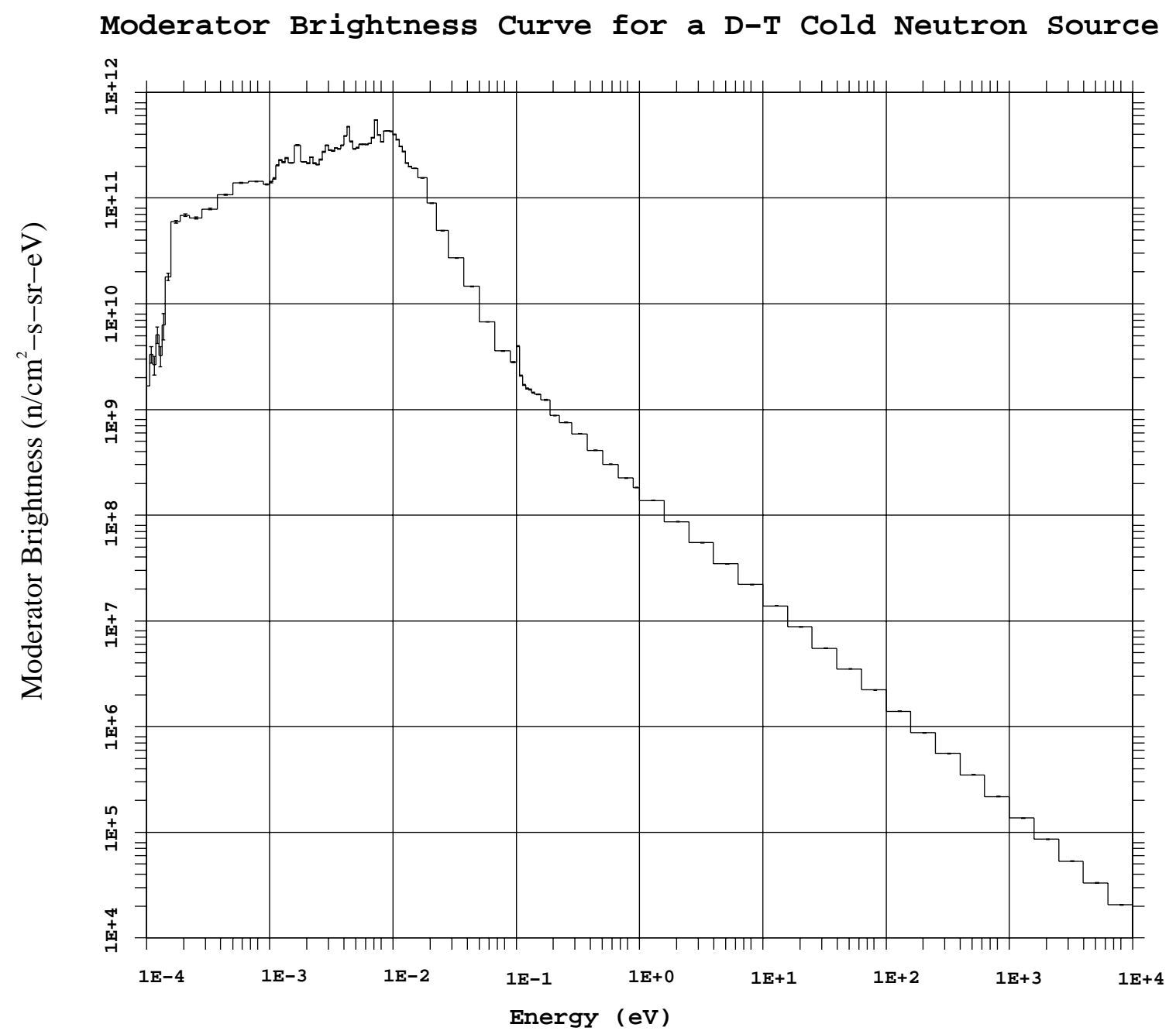

Figure 5.3.1 Moderator brightness curve for a D-T cold neutron source 
spectrum curve is not smooth. This is due to the ${ }^{9} \mathrm{Be}(\mathrm{n}, 2 \mathrm{n})$ reactions with the fast neutrons.

The $(n, 2 n)$ reaction of Be has a very small threshold energy as shown in Figure 5.3.2. Because the source neutron energy is $14 \mathrm{MeV}$, some neutrons are produced by this $(\mathrm{n}, 2 \mathrm{n})$ reaction far away from the source. These neutrons usually have lower energy. The neutrons produced by the $(\mathrm{n}, 2 \mathrm{n})$ reaction spread the neutron pulse width with spikes on the time emission spectrum because these neutrons born far away from the source at a later time reach the cryogenic moderator and contribute to the time emission spectrum. Therefore, the outer part of the beryllium reflector should be replaced with other materials such as graphite. Graphite is a good choice because it has a high density and a large inelastic cross section for fast neutrons (Figure 5.3.2). The absorption cross section of carbon is also smaller than that of beryllium for neutron energy between 1 and $7 \mathrm{MeV}$

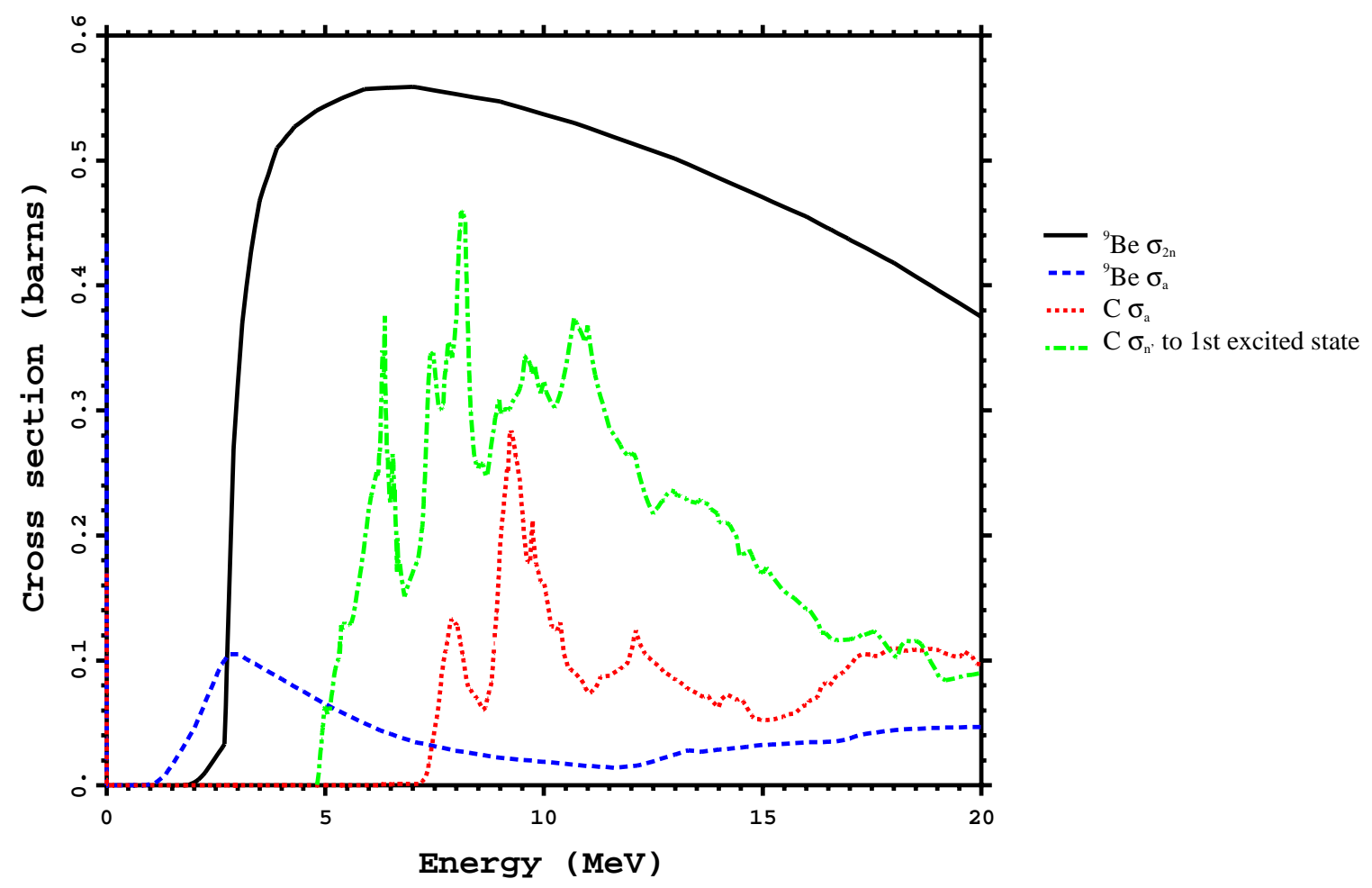

Figure 5.3.2 Be and C neutron reaction cross sections from ENDF60 
while the absorption cross section of carbon is much higher than that of beryllium for neutron energy above $7 \mathrm{MeV}$. With a graphite reflector replacing part of the beryllium reflector, neutrons with energy $>7 \mathrm{MeV}$ have a high probability to be absorbed by or slowed down to energy below the threshold energy of ${ }^{9} \mathrm{Be}(\mathrm{n}, 2 \mathrm{n})$ reaction. There is no simulation run for a system with graphite reflector. But, the change of the original moderator design for the Low Energy Neutron Source (LENS) implies that the beryllium reflector cannot be too large.

The exact neutron flux at the sample cannot be calculated with MCNP because neutron guides are usually incorporated into a cold neutron source. A computer code for neutron optics is needed to simulate the flux at the sample position. Neutrons may be totally reflected by a scattering material when the scattering length of the material is positive. The refractive index, $n$, of the scattering system for thermal neutrons is given by:[17]

$$
n=1-\frac{1}{2 \pi} \rho \lambda^{2} b
$$

where $\quad \rho$ is the number of nuclei per unit volume.

$\lambda$ is the wavelength of the incident neutrons.

$b$ is the coherent scattering length of the nuclei.

$b$ can be related to the cross-section by:[20]

$$
\sigma=4 \pi b^{2}
$$

The total reflection occurs when the glancing angle, $\theta$, is less than the critical value: 


$$
\theta_{c}=\cos ^{-1} n \approx \lambda \sqrt{\frac{\rho b}{\pi}}=\lambda \gamma_{c} \text { for } n \text { is close to } 1 .
$$

where $\quad \gamma_{c}$ is the critical angle per unit wavelength.

For example, natural Ni has a coherent scattering length of $10^{-14} \mathrm{~m}$ and an atomic density of $9 \times 10^{28} \mathrm{~m}^{-3}$. The critical glancing angle is $0.1^{\circ}$ (i.e. $6^{\prime}$ ) for $1 \AA$ neutrons. Thin silicon-wafer substrates can be coated with $\mathrm{Ni} / \mathrm{Ti}$ layers to make supermirrors to bend or guide the cold and thermal neutrons out of the cold moderator. Supermirrors can be used to bend the path of cold neutrons, collect the cold neutrons from a large moderator area, and focus the beam. For supermirror, $m$ is a number to describe its critical angle. $m$ is defined as the ratio of the critical angle for natural $\mathrm{Ni}$ and the critical angle for the supermirror.

\subsection{Summary of fusion-based neutron sources for neutron scattering}

A D-T fusion-based neutron source for thermal neutron scattering is shown to have a thermal neutron flux of $7.3 \times 10^{5} \mathrm{n} / \mathrm{cm}^{2} \mathrm{~s}$ at the sample position. The study shows that the fusion-based neutron source is more suitable for cold neutron scattering because of its long ion beam pulse. The long ion beam pulse is necessary for a fusion-based neutron source because the low duty factor requires much higher current drawn from the source during the pulse. A more practical ion pulse width is $50 \mu$ s. The flight path for a cold neutron source can also be shorter than the flight path of HIPPO diffractometer at LANSCE. Thus, the system can be operating at a high operating frequency with time choppers to allow counting only neutrons within certain energy range. The flux at the sample for LANSCE is $\sim 1 \times 10^{7} \mathrm{n} / \mathrm{cm}^{2} \mathrm{~s}$ when the source is operating at $70 \mu \mathrm{A}$. This flux 
is approximately 13 times of the flux achievable with the fusion-based neutron source. With a highly efficient diffractometer such as HIPPO, the count rate is comparable with LANSCE with a less efficient diffractometer. Therefore, the fusion-based neutron source can be considered as an economical neutron source for neutron scattering experiments at universities. 


\section{Chapter 6}

\section{Fission Multipliers for D-D/D-T Neutron Generators}

\subsection{Introduction}

Although the new fusion-based neutron generators currently under development have a potential yield of $10^{12} \mathrm{D}-\mathrm{D} \mathrm{n} / \mathrm{s}$ and $10^{14} \mathrm{D}-\mathrm{T} \mathrm{n} / \mathrm{s}$, some applications require higher flux that is available from nuclear reactors. This study attempts to design a subcritical fission multiplier with a $k_{\text {eff }}$ of 0.98 that will be coupled with compact neutron generators. There are two applications chosen in this study to show the gain in flux due to the use of fission multipliers - in-core irradiation and out-of-core irradiation. The idea of using a fission multiplier is not new; its concept is similar to the accelerator-driven systems (ADS) for transmutation of nuclear waste. The D-D/D-T neutron driven fission multiplier has been designed for applications that require a high neutron yield in the range of $10^{14}$ to $10^{16} \mathrm{n} / \mathrm{s}$. The first application of the neutron generator in this study is for positron production. Thermal neutrons are captured in ${ }^{113} \mathrm{Cd}$ with the emission of $\gamma$-rays and the generation of positrons in the pair production process.[21] The system for positron 
production described in Reference [21] consists of a $6.5 \mathrm{~cm}$ diameter ${ }^{113} \mathrm{Cd}$ cap, which is $6.5 \mathrm{~cm}$ long, with platinum plates inside. Thermal neutrons are captured by a ${ }^{113} \mathrm{Cd}$ cap and high-energy $\gamma$-rays are emitted. Positrons are produced in the platinum foils inside the ${ }^{113} \mathrm{Cd}$ cap by absorption of these $\gamma$-rays in the pair production process. The platinum foils also act as a positron moderator because of its negative work function of $-1.8 \mathrm{eV}$ in polycrystalline platinum.[22] In this study, the ${ }^{113} \mathrm{Cd}$ cap is placed very close to a subcritical pile driven by a D-T neutron generator. The discussion presented here is limited to the parameters that affect the optimization of thermal neutron absorption rate in the ${ }^{113} \mathrm{Cd}$ cap.

The second application studied is for Boron Neutron Capture Therapy (BNCT). It has been shown that a yield of $10^{14} \mathrm{n} / \mathrm{s}$ from a D-T neutron source is necessary to achieve a BNCT treatment time of $\sim 45$ minutes.[23] However, due to the public concerns of using tritium-based source in a hospital environment, there is an interest to study the use of a subcritical pile to boost the neutron yield from a D-D neutron generator to at least $5 \times 10^{13} \mathrm{n} / \mathrm{s}$. Unlike fission converter plates that convert the thermal neutrons in a research reactor to fast neutrons, a subcritical fission multiplier is designed to increase the flux of a neutron source. Because most neutrons produced in a reactor are thermalized when they exit the reactor core, it is necessary to convert these thermal neutrons into fast neutrons and re-moderate them to epithermal energy range.[24] A fission converter assembly with a $k_{\text {eff }}$ of 0.26 was shown to be capable of producing $9 \times 10^{9} \mathrm{n} / \mathrm{cm}^{2} \mathrm{~s}$ in a $10 \mathrm{MW}$ research reactor. A $k_{\text {eff }}$ of 0.26 would be sufficient for the fission converter plates because they are irradiated with a high thermal neutron flux. A fission multiplier, on the other hand, 
usually requires a $k_{\text {eff }}$ close to 1 because its goal is to produce more neutrons. A $k_{\text {eff }}$ of 0.98 was chosen in this study.

These two applications that we decided to study belong to two different categories: (a) in-core irradiation and (b) out-of-core irradiation. In an ideal situation, the total number of fission neutrons generated by a source neutron in a fission multiplier with $k_{\text {eff }}$ of 0.98 is $\sim 50$ for in-core irradiation and $\sim 30$ for out-of-core irradiation. For in-core irradiation, the sample being irradiated is placed inside or in the vicinity of the multiplier

assembly. For example, the ${ }^{113} \mathrm{Cd}$ cap of a positron production system is located very close to the fuel. One example of in-core irradiation is radioisotope production. For outof-core irradiation, the neutron beam is extracted out of the multiplier assembly, such as in the case of neutron radiography, as well as BNCT. In this study, the advantages of the fission-multiplier enhanced D-D neutron generator system for BNCT are analyzed because it requires an epithermal neutron beam that is not readily available in most reactors without contamination from thermal and/or fast neutrons.

\subsection{Source brightness and net multiplication}

The major factors that influence the effectiveness of a fission multiplier in improving the neutron flux are source brightness and the fuel material. Any neutron source can be designed to produce a lot of neutrons. However, a high neutron yield is not the only factor that determines the usefulness of a particular neutron source. An important parameter is the neutron flux. The neutron flux is defined as the number of neutrons crossing a unit area per unit time. i.e.:

$$
\Phi=n v
$$


where $\quad n$ is the neutron density.

$v$ is the velocity of neutron.

A neutron source with a high yield must also have a high neutron density in order to provide a high neutron flux. When the neutron generator is coupled with a fission multiplier, the volume of the source increases. As a result, the neutron density increases slower than the yield and the neutron flux will not be improved linearly with the true system multiplication factor. The definition of "true system multiplication" taken from the user manual of MCNP is:[14]

$$
1+k_{e f f}+k_{e f f}^{2}+\cdots+k_{e f f}^{n}+\cdots=\frac{1}{1-k_{e f f}}
$$

The "true system multiplication" includes the neutrons that are absorbed in the fission process. Due to its name, some confusion may arise: that an optimized fission multiplier has a net gain in neutron flux equal to the "true system multiplication" if the source brightness (i.e. number of source neutrons generated in a unit volume per unit time) is not reduced as a consequence of introducing a subcritical pile.[25] The net multiplication, $M$, for a fixed source problem in MCNP is defined as:

$$
M=1+G_{f}+G_{x}=W_{e}+W_{c}
$$

where $\quad G_{f}$ is the weight of gain from fission.

$G_{x}$ is the weight of gain from $\mathrm{n}, \mathrm{xn}$ reaction.

$W_{e}$ is the weight of escape from the system.

$W_{c}$ is the weight of capture in the system.

$$
G_{f}=(\bar{v}-1) \times W_{f}
$$

where $\quad W_{f}$ is the weight of fission. 
$\bar{v}$ is the average number of fission neutrons per fission.

The net multiplication, $M$, takes into account the neutrons undergoing fission and capture in the system. If $M$ is fixed, $W_{e}$ increases when $W_{c}$ decreases. $W_{c}$ includes all absorption processes in a fixed source problem except the fission process. For a system with a $k_{\text {eff }}$ of $0.98, M$ is approximately 30 for a core loaded with $100 \%{ }^{235} \mathrm{U}$ and 33 for a core loaded with reactor-grade $\mathrm{Pu}$ only. There is a minimum $W_{c}$ even in a perfect system because neutrons are absorbed in the fuel by other process. By assuming that a fission multiplier does not reduce the source brightness significantly, the maximum $W_{e}$ strongly depends on $M$ and $W_{c}$ of the fuel if the absorption in the moderator and structure materials is negligible.

\subsection{Designs of fission multipliers}

From the discussion in the previous section, several key issues in optimizing a subcritical fission multiplier are identified:

1. The fuel should have a high content of fissile material in order to reduce the absorption in the fuel. (i.e. no ${ }^{238} \mathrm{U}$ )

2. The average number of fission neutrons per fission for the fissile material should be large in order to minimize the number of neutrons that undergo fission.

3. The size of the fission multiplier should be minimal with high leakage directed toward the sample being irradiated. In other words, the sample should see a fuel assembly that is optically thin.

4. The moderator and other structure materials should not have large absorption crosssections so that the neutrons born in the fuel can escape the core. 


\subsubsection{In-core irradiation - Positron production system}

Because plutonium has a larger average number of fission neutrons per fission than uranium, the fuel chosen in this study is $\mathrm{Pu}-10 \mathrm{Zr}$. Reactor-grade $\mathrm{Pu}$ with an isotope composition of $2 \%{ }^{238} \mathrm{Pu}, 56 \%{ }^{239} \mathrm{Pu}, 26 \%{ }^{240} \mathrm{Pu}, 11 \%{ }^{241} \mathrm{Pu}$ and $5 \%{ }^{242} \mathrm{Pu}$ by weight is used. Metallic fuel is chosen for its high Pu density. Table 6.3.1 shows other specification for the fuel rod. The total Pu loading of the subcritical assembly is $18.7 \mathrm{Kg}$.

\begin{tabular}{|l|l|}
\hline Fuel & Metallic Pu-10Zr \\
\hline Density & $14.36 \mathrm{~g} / \mathrm{cm}^{3}$ \\
\hline Pu density & $12.9 \mathrm{~g} / \mathrm{cm}^{3}$ \\
\hline Fuel temperature & $755{ }^{\circ} \mathrm{K}$ \\
\hline Pu composition & $\begin{array}{l}2 \%{ }^{238} \mathrm{Pu}, 56 \%{ }^{239} \mathrm{Pu}, 26 \%{ }^{240} \mathrm{Pu}, 11 \%{ }^{241} \mathrm{Pu}, \\
5 \%{ }^{242} \mathrm{Pu}\end{array}$ \\
\hline Diameter & $0.63 \mathrm{~cm}$ \\
\hline Length & $17.25 \mathrm{~cm}$ \\
\hline Plenum & $5.75 \mathrm{~cm}$ \\
\hline Pin arrangement & $\mathrm{Hexagonal}$ pitch \\
\hline Pitch & $1 \mathrm{~cm}$ \\
\hline Number of pins & 270 \\
\hline Gap & $\mathrm{Na} \mathrm{filled}$ \\
\hline Thickness & $0.2 \mathrm{~mm}$ \\
\hline Clad & $\mathrm{HT}-9$ \\
\hline Density & $7.7 \mathrm{~g} / \mathrm{cm}^{3}$ \\
\hline Thickness & $0.4 \mathrm{~mm}$ \\
\hline Composition & $84.49 \% \mathrm{Fe}, 11.8 \% \mathrm{Cr}, 0.51 \% \mathrm{Ni}$, \\
\hline & $1.03 \% \mathrm{Mo}, 0.4 \% \mathrm{Mn}, 0.33 \% \mathrm{~V}, 0.52 \% \mathrm{~W}$, \\
\hline
\end{tabular}

Table 6.3.1 Specification for the fuel and clad used in D-T driven fission multiplier 
The cladding material is chosen to be martensitic stainless steel, HT-9, for its excellent strength and swelling properties under fast neutron irradiation.[26] Helium at a pressure of $2.3 \mathrm{MPa}$ is assumed to be the coolant for the subcritical assembly due to its low absorption cross-section for neutron. Beryllium is used as moderator and reflector. A beryllium moderator is used rather than a light water moderator because the light water tends to over-thermalize the neutrons before they reach the Cd cap. A secondary light water moderator surrounding the $\mathrm{Cd}$ cap is used to enhance the thermal neutron flux in the Cd cap. (Figure 6.3.1)

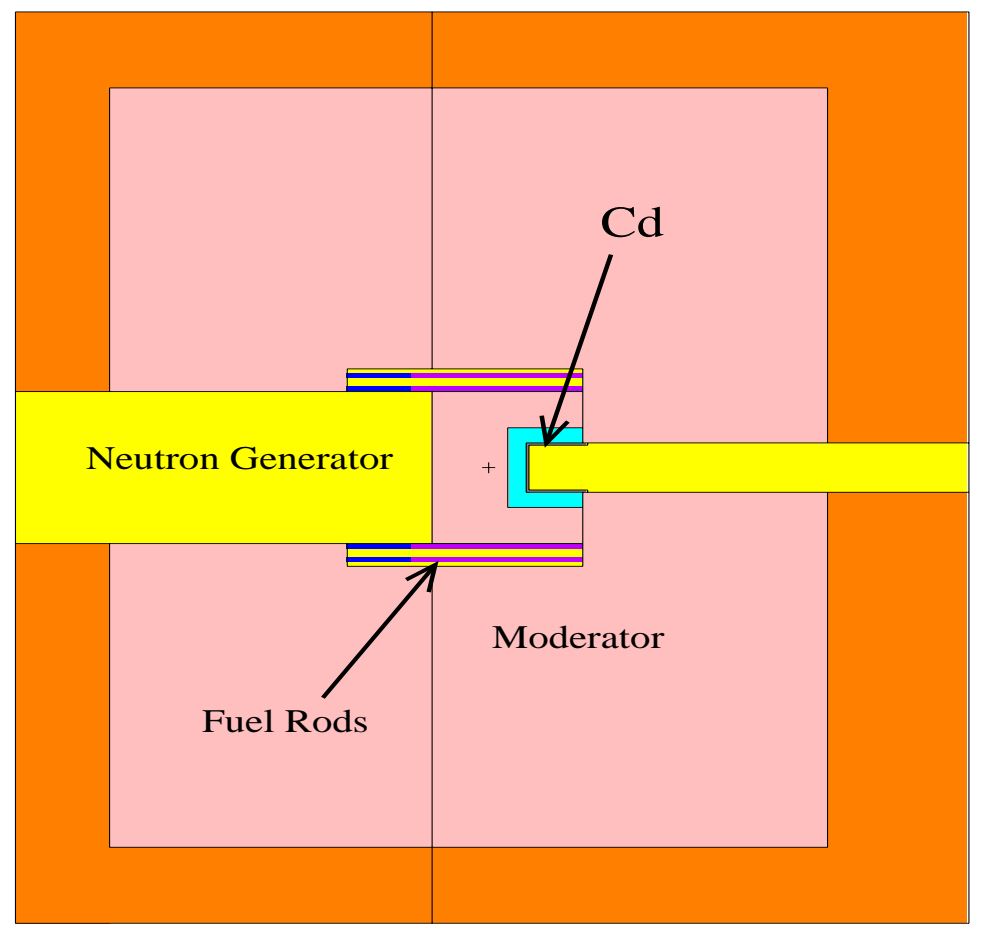

Figure 6.3.1 A D-T driven fission multiplier showing a Cd cap surrounded by a small light water moderator

The coaxial neutron generator has a diameter of $20 \mathrm{~cm}$ and a length of $8 \mathrm{~cm}$. A cylindrical fuel assembly surrounds the neutron generator and the Cd cap. Its inner diameter and outer diameter are $20 \mathrm{~cm}$ and $27.4 \mathrm{~cm}$, respectively. As shown in Figure 
6.3.2, the fuel assembly is consisted of 270 fuel pins. Each of these fuel pins has a plenum filled with sodium to allow for thermal expansion of the fuel pellets.

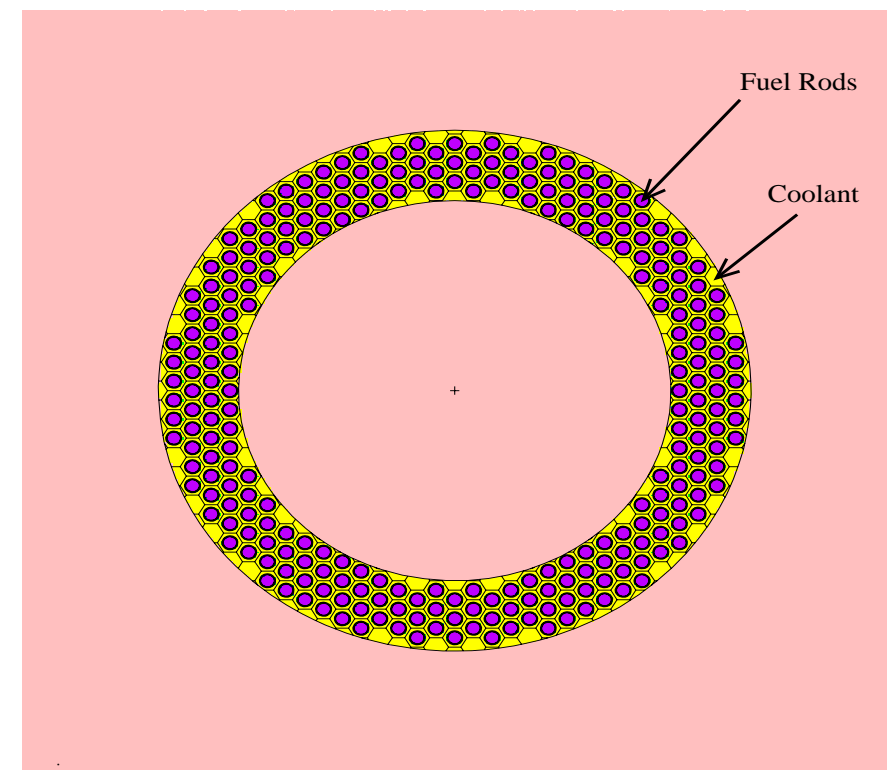

Figure 6.3.2 A cylindrical fuel assembly for the D-T fission multiplier

The calculation is performed using MCNP. There are two modes of neutronics calculation in MCNP: (a) fixed source and (b) criticality. In most cases, when the $k_{\text {eff }}$ of a system is close to 1 , a criticality problem has almost the same result as a corresponding fixed-source problem. Tracking one history of a fixed source problem with $k_{\text {eff }}$ close to 1 can sometimes take a long time to finish. Therefore, the calculation is performed with criticality calculation first. Then, it is verified with the fixed source calculation to account for the source neutron spectrum.

\subsubsection{Out-of-core irradiation - BNCT}

In designing a beam shaping assembly for BNCT, aluminum, iron and aluminum fluoride are some of the best moderator materials. The U-Mo alloys have a very high fuel density.[27] The U-10Mo alloy fuel has a density of $16.8 \mathrm{~g} / \mathrm{cm}^{3}$. The alloy fuel particles, 
when dispersed in an aluminum matrix (i.e. the moderator), can act as a high brightness fission neutron source. Table 6.3.2 shows some physical properties of U-10Mo alloy dispersed in an aluminum matrix. The fuel is assumed to be $100 \%$ enriched ${ }^{235} \mathrm{U}$. Pu is not chosen here because U-10Mo shows stable behavior with respect to fuel-matrix interaction in the form of inter-diffusion.[28]

\begin{tabular}{|l|l|}
\hline Fuel composition & $90 \%{ }^{235} \mathrm{U}, 10 \%$ Mo \\
\hline Aluminum matrix volume (\%) & 71 \\
\hline Fuel volume (\%) & 29 \\
\hline Fuel density & $4.4 \mathrm{~g}-\mathrm{U} / \mathrm{cm}^{3}$ \\
\hline
\end{tabular}

Table 6.3.2 Physical properties of U-10Mo alloy aluminum matrix dispersion fuel

Because the fuel is dispersed in the moderator, the change in moderator size is minimal. The Beam Shaping Assembly (BSA) for D-D neutron source is taken from a BNCT system optimized for liver cancer treatment with D-D fusion neutrons. The back of its Fe moderator is replaced with the dispersion fuel as shown in Figure 6.3.3. A power of $\sim 630 \mathrm{~W}$ is calculated for this multiplier from the neutron yield of the D-D neutron generator and the $k_{\text {eff }}$ of the fission multiplier. Therefore, for simplicity and reduction of computer time, there is no coolant channel in this computational model.

A conical D-D neutron generator is used for the BNCT system. The conical neutron generator is another design under development at LBNL. It has a diameter of 16 $\mathrm{cm}$ and a length of $32 \mathrm{~cm}$. The iron moderator has a dimension of $80 \mathrm{~cm} \times 25.5 \mathrm{~cm} \times 40$ $\mathrm{cm}$. The fuel meat has a dimension of $80 \mathrm{~cm} \times 14.5 \mathrm{~cm} \times 40 \mathrm{~cm}$. The total U loading is $180 \mathrm{Kg}$. The moderator for the epithermal neutron beam is made of Fluental ${ }^{\mathrm{TM}}$ with a thickness of $27 \mathrm{~cm}$. 


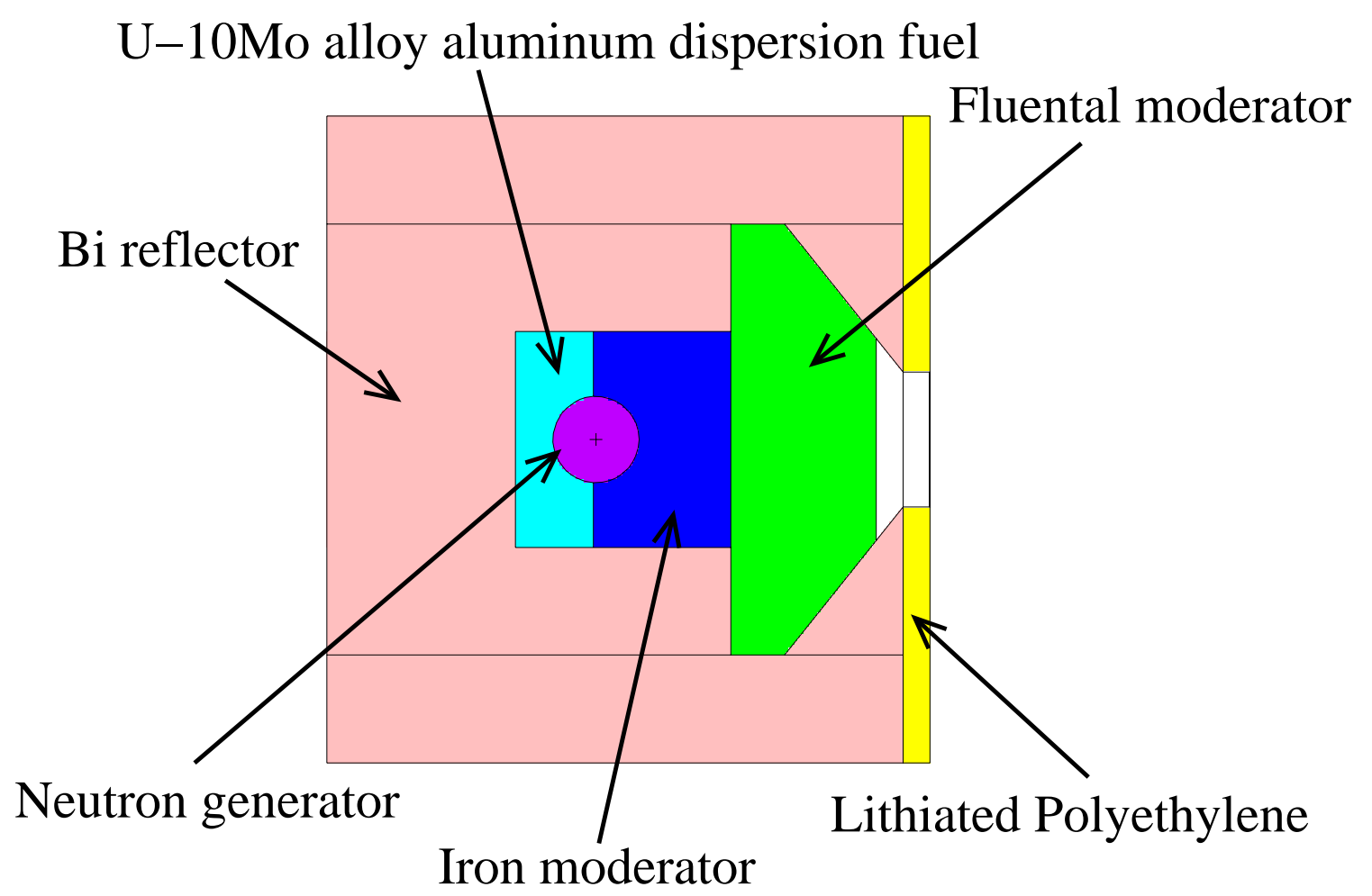

Figure 6.3.3 A D-D neutron driven fission multiplier with metal alloy fuel dispersed in its moderator

\subsection{Results and Discussion}

The fission multipliers for the positron production system and BNCT have a $k_{\text {eff }}$ of 0.98449 and 0.98028 respectively. Although the "true system multiplication" is $1 /(1-0.98449)=64.5$, the absorption rate in the Cd cap of the positron production system is increased by a factor of 25 only. This number shows that the "true system multiplication" does not represent the gain in neutron flux inside the core of a fission multiplier. In fact, if light water is being irradiated with neutrons to produce heavy water, the absorption rate of neutrons in light water should never be expected to increase more than $G_{f}$. When the fuel rods are removed to reduce the volume of the whole system, the flux being seen by the light water increases. This example provides a simple analogue to the positron production system. Not only the net gain in the neutron absorption rate of the 
Cd cap is always less than the "true system multiplication" or the net multiplication, $M$, but also it is always less than the difference between $M$ and $W_{c}$ in the fuel. The result shows that $M$ and $W_{c}$ for the fuel in the positron production system are 43.1 and 8.3, respectively. Therefore, the true theoretical maximum gain is 34.8 for the whole system. $G_{f}$ for the positron production system is 35.0 so $G_{x}=\left(M-1-G_{f}\right)=7.1$ is due to the $\operatorname{Be}(\mathrm{n}, 2 \mathrm{n})$ reaction. Therefore, the theoretical maximum net gain due to the fission multiplier is $(34.8-7.1)=27.7$ which is close to 25 . The remaining $10 \%$ discrepancy is due to the neutron absorption in structure material and source brightness.

The fission multiplier for BNCT has a relatively lower gain because the source brightness does not increase as much as the total neutron yield. The net gain in neutron flux at the beam port exit is $\sim 17.5$ as shown in Figure 6.4.1. $M$ and $G_{f}$ of the D-D neutron generator driven fission multiplier for BNCT are 31.0 and 29.9, respectively. The gain from $(\mathrm{n}, \mathrm{xn})$ reaction in this system is comparatively small. $G_{x}$ and $W_{c}$ in the fuel are $<0.1$ and 3.6 respectively. A theoretical maximum gain of 27.3 is expected. Although ${ }^{235} \mathrm{U}$ on average produces less fission neutrons than reactor-grade $\mathrm{Pu}$, the neutron absorption cross-section for ${ }^{235} \mathrm{U}$ is smaller than reactor-grade Pu. Therefore, it is not necessary to use $\mathrm{Pu}$ in order to get the maximum gain.

The large fuel meat at the back of the neutron generator can explain why the gain in neutron brightness is smaller than its yield. The dispersion fuel meat can be separated into moderator and a high-density metallic fuel assembly to reduce the fission neutron source volume and improve the source brightness. However, if the fuel assembly is placed too close to the neutron generator, a thicker moderator is needed because there is a significant number of fission neutrons with energy higher than $2.5 \mathrm{MeV}$. When the 


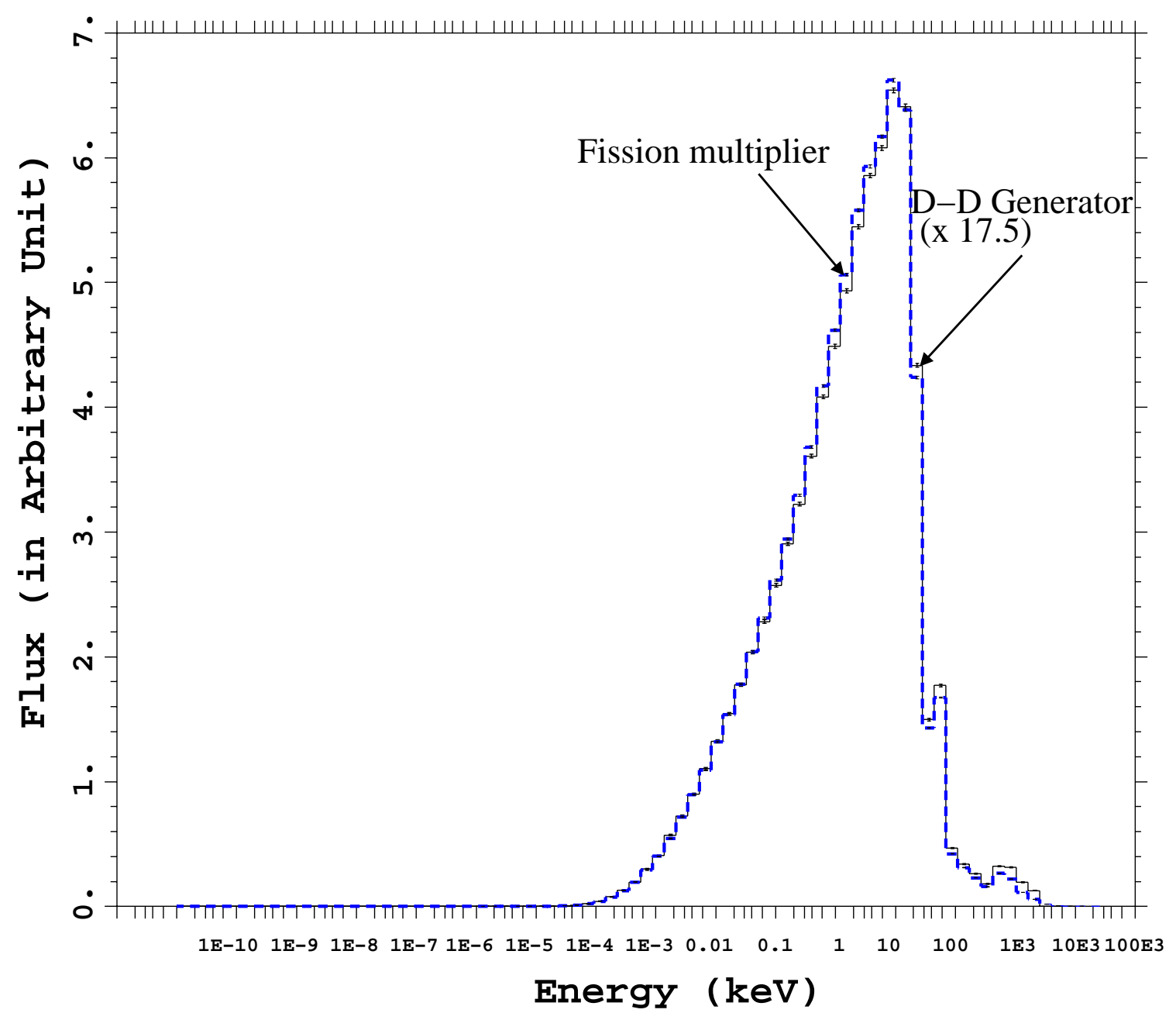

Figure 6.4.1 Epithermal neutron flux at the beam port exit of the BNCT system (Fission multiplier vs. D-D neutron generator)

moderator size is increased, the geometry factor will also reduce the gain in flux at the beam port exit. If the fuel is placed far from the generator, the size of the subcritical assembly also increases and the brightness also reduces due to the geometric factor. Therefore, the fission neutrons with energy higher than $2.5 \mathrm{MeV}$ are the issues in obtaining the maximum gain in this system because they require a thicker moderator. 


\subsection{Summary of fission multiplier}

The FRM-II reactor described in Reference [21] has a peak thermal neutron flux of $8 \times 10^{14} \mathrm{n} / \mathrm{cm}^{2} \mathrm{~s}$. The core loading for this reactor is $7.5 \mathrm{~kg}$. The subcritical multipliers have a core loading of $18.7 \mathrm{~kg}$ and $180 \mathrm{~kg}$ depending on the type of fuel being used. The fuel for these systems is also either highly enriched uranium or reactor-grade Pu without

${ }^{238} \mathrm{U}$. If low-enriched uranium is used, the gain from fission is significantly reduced because many fission neutrons are absorbed in ${ }^{238} \mathrm{U}$ and the fuel assembly volume increases. If the subcritical assembly for the positron production system is fueled with $\mathrm{U}-19 \mathrm{Pu}-10 \mathrm{Zr}$, the absorption rate is reduced by $50 \%$ due to the reduction in source brightness and ${ }^{238} \mathrm{U}$. Using a light water moderator or having a low leakage design can reduce the uranium core loading. Unfortunately, these approaches will also significantly reduce the number of neutrons useful for the applications. The same situation will not happen to a reactor because the flux in a reactor is controlled by the power. The flux from a subcritical multiplier depends on the neutron yield of the neutron generator only. In conclusion, we have shown that a gain of $\sim 25$ times can be achieved in a positron production system using D-T generator. For the out-of -core irradiation, a gain of $\sim 17$ times is obtained in Boron Neutron Capture Therapy (BNCT) using a D-D neutron generator. The total number of fission neutrons generated by a source neutron in a fission multiplier with $k_{\text {eff }}$ is $\sim 50$. For the out-of-core irradiation, the theoretical maximum net multiplications is $\sim 30$ due to the absorption of neutrons in the fuel. 


\section{Chapter 7}

\section{Other possible applications of fusion-based neutron generators}

\subsection{A review of neutron sources for contraband detection}

In response to the September $11^{\text {th }}$ terrorist attack, a scoping study of using the accelerator-based neutron generators for explosive detection in the airport is carried out. The primary design goal is to detect explosives listed in Table 7.1.1. A detection limit of $1 \mathrm{lb}$ is set as a requirement for this system. The system should also be capable of detecting the elemental compositions. Standard X-ray machines can only detect the shape and the density of an object. When the explosives are rolled into thin sheets or in liquid forms, X-ray machines fail to detect them because the attenuation of photons is a function of charge density. 


\begin{tabular}{|l|l|}
\hline Explosive & Composition \\
\hline ANFO $^{2}$ (Ammonium nitrate fuel oil) & $45 \% \mathrm{H}, 8 \% \mathrm{C}, 19 \% \mathrm{~N}, 28 \% \mathrm{O}$ \\
\hline Black Powder & $75 \% \mathrm{KNO}_{3}, 15 \% \mathrm{C}, 10 \% \mathrm{~S}$ \\
\hline $\mathrm{C} 4$ (Composition 4) & $33 \% \mathrm{H}, 21 \% \mathrm{C}, 23 \% \mathrm{~N}, 23 \% \mathrm{O}$ \\
\hline Dynamite & $41 \% \mathrm{H}, 10 \% \mathrm{C}, 18 \% \mathrm{~N}, 31 \% \mathrm{O}$ \\
\hline PETN & $28 \% \mathrm{H}, 17 \% \mathrm{C}, 14 \% \mathrm{~N}, 41 \% \mathrm{O}$ \\
\hline RDX & $28.57 \% \mathrm{H}, 14.29 \% \mathrm{C}, 28.57 \% \mathrm{~N}, 28.57 \% \mathrm{O}$ \\
\hline SemtexH & $36 \% \mathrm{H}, 23 \% \mathrm{C}, 18 \% \mathrm{~N}, 23 \% \mathrm{O}$ \\
\hline Smokeless Powder & $26 \% \mathrm{H}, 22 \% \mathrm{C}, 11 \% \mathrm{~N}, 41 \% \mathrm{O}$ \\
\hline
\end{tabular}

Table 7.1.1 Some common explosives and their compositions

Unique characteristics of neutron interactions allow distinguishing different elements with different reaction rates. Neutrons can also induce secondary characteristic radiation, which can be used as a signature of elements and compounds. There are many approaches proposed by different groups to use neutrons for screening small luggages. These approaches are discussed in the following sections. The Federal Aviation Administration (FAA) wants to have a screening system that can provide an image, density and elemental compositions of the inspected objects. The mass of the whole system cannot be more than 12 tonnes. A probability of detection of more than $95 \%$ is required. The processing rate is $8 \mathrm{sec}$ per luggage or faster. The cost for each machine should be less than $\$ 1 \mathrm{M}$.

\subsubsection{Thermal Neutron Analysis (TNA)}

In Thermal Neutron Analysis (TNA), a luggage is irradiated with thermal neutrons. The objects in the luggage absorb some of the neutrons and the $(n, \gamma)$ reactions with the nuclides in these objects produce a gamma spectrum with a signature

2 Emulsion, Tovex and slurry are aqueous version of ANFO.

3 Composition is given for NG dynamite. Other common dynamites are AN Gel, ExGel, and Unigel. 
characterizing the elemental compositions in the luggage.[29] Radioactive ${ }^{252} \mathrm{Cf}$ and D-D neutron generator had been used as neutron sources for TNA.[30] This technique has several problems. It cannot be used to detect $\mathrm{C}$ and $\mathrm{O}$ because the neutron absorption cross-sections for $\mathrm{C}$ and $\mathrm{O}$ are too small. Hydrogen may be detected only when the moderator is not made of hydrogenous material. As hydrogen is the most effective nuclide in thermalizing the neutrons, a non-hydrogenous moderator will be larger in size and weight. It also can produce any image of the object being interrogated. Pin-hole imaging or projection imaging techniques fail in TNA because the energy of the gamma rays coming from the $\mathrm{N}(\mathrm{n}, \gamma)$ reactions is $10.8 \mathrm{MeV}$ and thermal neutrons diffuse randomly in the luggage. It is difficult to collimate or shield the gamma rays at this high gamma energy. Even if the hydrogen and nitrogen ratio can be found, the inability of producing an image and spatial density map creates a lot of false alarms because a lot of materials (e.g. leather and meat) contain nitrogen and hydrogen. Therefore, people can only use TNA to identify small objects. For example, a pair of shoes can be irradiated. With the aid of intelligent computer software, hidden explosives in the shoes can be spotted if the operator tells the computer that the object is a pair of shoes. This computer program relies on a large database of different objects.

\subsubsection{Fast Neutron Analysis (FNA)}

The Fast Neutron Analysis (FNA) technique use pulsed D-T (14 MeV) neutrons rather than thermal neutrons. Therefore, the mechanism of how it works is slightly different from TNA. When a fast neutron collides with a nuclide, it leaves the nuclide in its excited states. The excited nuclide emits gamma rays as a process of de-excitation. 
These gamma rays due to inelastic neutron scattering can be used as fingerprints for different elements. When the pulsed D-T neutron beam is collimated, the position of the inelastic scattering reaction sites can be determined from the time of flight. However, the collimation usually produces a significant amount of noise to the detector so heavy shielding is required. The angular divergence of the collimated neutron beam also limits the flux and resolution of the system. In general, its spatial resolution is barely acceptable. Furthermore, its massive shielding requires modification of the building structure. Time-gated TNA technique has been combined with FNA for non-intrusive inspection of drugs and explosives in small vehicles and maritime vessels.[31] However, this combined approach is good for bulk explosive and its result heavily relies on the algorithm refinement. For these reasons, it is expensive and difficult to adopt this technique in an airport environment.

\subsubsection{Associated Particle Imaging (API)}

FNA requires a high neutron yield and produces a high noise signal. To address these two problems, a seal-tube D-T neutron generator can have a 2-D position sensitive charge particle detector inside the neutron generator to tag each neutron's direction by its associated ${ }^{4} \mathrm{He}$ particle in the fusion reaction. The obtained precise neutron flight direction and flight time allow the production of a tomographic image for each nuclide. The image produced by this technique is excellent because the noise due to collimation is eliminated. However, the counting time for recognizing $1 \mathrm{lb}$ of substance is $\sim 1$ minute because the detector for the associated ${ }^{4} \mathrm{He}$ particles has a very high count rate for high neutron yield.[32] This screening time is too long if this technique is applied to screen every luggage in an airport. 


\subsubsection{Pulsed Fast Neutron Spectroscopy (PFNS)}

Pulsed Fast Neutron Spectroscopy (PFNS), sometimes also called Pulsed Fast Neutron Transmission Spectroscopy (PFNTS) use a white neutron source. It measures the transmission of fast neutron with different energy. A projection image is obtained from the transmitted fast neutron hitting on a 2-D proton recoil detector array. Because the inelastic scattering and total removal cross-sections have different resonance peaks for different elements, the density and composition of an object can be determined.[33] The white neutron spectrum can be produced by a cyclotron accelerating $\mathrm{D}^{+}$ions onto a $\mathrm{Be}$ target.[34]

Currently, this system does not fulfill the requirements set forth by FAA.[35] There are several technical problems that need to be addressed for this approach. The shielding is too heavy for the current building structure. It requires a source neutron pulse width of a few nanoseconds in order to obtain better energy resolution. Because the neutron energy is measured by time of flight (ToF) technique, the pulse width must be small enough to obtain a reasonable resolution. For fast neutrons with energy of a few $\mathrm{MeV}$, the flight time is only hundreds of nanoseconds depending on the neutron energy and the length of flight path. A T-T neutron source can substitute the $\mathrm{Be}(\mathrm{d}, \mathrm{n})$ cyclotron neutron source to obtain shorter source neutron pulse width. It can also lower the cost.

\subsubsection{Nuclear Resonance Radiography (NRR)}

The working principle behind Nuclear Resonance Radiography (NRR) is basically the same as PFNS except that it uses a variable monoenergetic D-D neutron source. The $\mathrm{D}^{+}$ions are accelerated to 2 to $6 \mathrm{MeV}$. The source neutron energy spectrum is 
a function of angle in this reaction. At zero degree, the neutrons have the highest energy. At larger angle, the neutron intensity is smaller and the counting time is longer. This problem can be solved if the accelerator can change the $\mathrm{D}^{+}$energy and only the neutrons emitted at zero degree are used. The energy of $\mathrm{D}^{+}$ions can be changed by turning on and off section(s) of an RFQ accelerator. By using such an expensive RFQ accelerator, the neutron energy of a monoenergetic neutron source can be varied. Otherwise, the counting time for the luggage at larger angle is much longer than at zero degree. The selected source neutron energies are resonance peaks and valleys for different elements so the elements in an object can be easily differentiated from the images of the same object taken with different neutron energy. The transmitted fast neutrons project a 2-D image on a scintillation screen. A mirror, a lens and a CCD camera are employed to record the image.[36] This technique does not require a source neutron pulse width of nanoseconds as PFNS does. However, the mass of the accelerator and shielding remains the issues in deploying it in an airport environment.

\subsubsection{Dual modality imaging}

Currently, no system can satisfy all the goals set forth by the FAA. However, the author believes that a hybrid TNA and X-ray system may achieve all these goals at a low cost by combining the advantages of thermal neutron and X-ray. An envisioned system is described after the discussion of a dual-energy X-ray imaging system.

Dual/Multiple-energy X-ray machines had been utilized in the airport. By using two or more different energies, these X-ray machines can distinguish organic, metal, or mixed objects when it encodes its images in pseudo colors. With more sophisticated algorithm and two almost identical images taken from slightly different angles at the 
same time, a stereoscopic color X-ray image can be produced.[37] However, this machine works only as good as the person who inspect the object with the X-ray images. Explosives may be hidden with special shapes since no information about the elemental composition of object is obtained. Because it is prone to human errors, a hybrid system may reduce the reliance on human inspection.

The disadvantage of a dual-energy X-ray can be complemented by neutrons activation analysis. The X-ray image can provide the spatial information of the objects inside a luggage. With the spatial information and density information, the TNA technique can be applied without any collimation for the $10.8 \mathrm{MeV} \gamma$-rays emitted by the ${ }^{14} \mathrm{~N}(\mathrm{n}, \gamma)$ reaction. An array of gamma detectors can be placed around the object. By taking the density map obtained from the X-ray image, the reaction rate in different objects due to thermal neutron irradiation can be calculated from the data collected by the gamma detectors. From the reaction rates with nitrogen and hydrogen, the density of $\mathrm{H}$ and $\mathrm{N}$ for each object in a luggage can be found. The collimation of the $10.8 \mathrm{MeV} \gamma$-ray usually reduces the efficiency and introduces noise to the gamma detectors. Because the envisioned system has eliminated the collimation, better counting efficiency is expected. The improvement in efficiency may lead to a lower neutron yield requirement. Therefore, the neutron shielding is minimized. Furthermore, the neutron source can be pulsed and time-gated to take advantage of the fast neutron and obtain the oxygen and carbon density.

A literature search on this type of systems shows that only a few studies were done on this approach. A similar system called XENIS (X-ray Enhanced Interrogation 
System) was tested in a number of airports to collect the critical data for evaluating a hybrid X-ray and TNA system.[38] A simplified version of this system, Portable Isotopic Neutron Spectroscopy (PINS), had been used to identify the chemical weapon agent inside unused rusting ammunitions.[39] The X-ray images of the warheads are used to distinguish the chemical weapon projectiles with a blasting tube down their centers. Then, PINS is used to identify the chemical agent filled the projectile cavities. Because the system for airport luggages has to deal with more than one object in the luggage at a time, multiple gamma detectors are used and the counts are measured with the luggage at different position on the conveyor belt. These data combined with the density map from an X-ray system will produce a complicated matrix equation that can be solved to find the elemental density of the objects. The working principle is similar to the medical “emission-transmission” systems which perform both X-ray transmission imaging to obtain anatomical information and radionuclide emission imaging to extract functional information.[40]

\subsection{Brachytherapy}

Brachytherapy is a type of radiation therapy in which radiation sources are placed directly into the tumor (interstitial brachytherapy) or into a body cavity in the vicinity of the tumor being treated (intracavity brachytherapy). The dose rate drops off rapidly as the distance increases from the source. Compared with an external beam irradiation, brachytherapy has the advantage of delivering a high dose to a localized volume and minimizing the radiation damage to normal tissues adjacent to the tumor. Conventionally, brachytherapy utilizes radioactive isotopes as radiation sources. Radioactive isotopes are usually encapsulated into small pellets or "seeds". For a low-dose-rate source with short 
half-life radioactive isotopes, the seeds can be permanently implanted into a patient. For a high-dose-rate source with longer half-life radioactive isotopes, the seeds are on the tip of needles, which are loaded into the tumor temporarily by a machine with shielding.

\subsubsection{Photon Brachytherapy}

The brachytherapy treatment in the United States commonly uses a gamma source with low photon energy. One advantage of a gamma source is comparatively easy fabrication of the seeds. Low energy photons also have the benefits of short range in tissue. Because the effectiveness of photon beams varies with the dose rate and dose delivered.[41] High-Dose-Rate (HDR) brachytherapy treatment is preferred. HDR brachytherapy is delivered by a remote after-loading device in order to avoid unnecessary occupational dose for the clinical personnel.

${ }^{137} \mathrm{Cs}$ and ${ }^{192} \mathrm{Ir}$ are two widely used gamma sources for brachytherapy. The photon energy for these conventional gamma sources ranges from 400 to $660 \mathrm{keV} .{ }^{103} \mathrm{Pd}$ and ${ }^{125} \mathrm{I}$ are examples of low energy gamma sources used in permanent implants of prostate tumors. The low photon energy has many advantages. It requires less shielding materials and spare more healthy tissue. The low photon energy enables the implantation short half-life seeds into a patient permanently. It also has higher relative biological effectiveness (RBE). RBE is defined as the ratio of the dose of some standard photon beam to the dose of the test beam that is necessary to produce the same level of biological effect. The test beam is usually ${ }^{60} \mathrm{Co}$ while some older reference photon beams are 250 $\mathrm{kV}_{\mathrm{p}}$ X-ray.[42] A recent study shows that the $\mathrm{RBE}$ value for ${ }^{192} \mathrm{Ir}$ is 1.3 relative to ${ }^{60} \mathrm{Co}$.[43] The mean photon energies of ${ }^{103} \mathrm{Pd},{ }^{125} \mathrm{I}$ and ${ }^{241} \mathrm{Am}$ are $21 \mathrm{keV}, 28 \mathrm{keV}$ and 60 $\mathrm{keV}$; their RBE values are 2.3, 2.1, and 2.1, respectively. 


\subsubsection{Neutron Brachytherapy}

The effectiveness of fast neutron brachytherapy in treating radioresistant tumors had been confirmed by the practice of implanting ${ }^{252} \mathrm{Cf}$ capsules into the patients for a short period of time.[44] A RBE value of 6 for low dose rate (LDR) irradiation with ${ }^{252} \mathrm{Cf}$ neutrons has been adopted for various tumor sites based on the clinical data from the University of Kentucky trial.[45] The primary cell killing mechanism of fast neutrons is the recoiled protons from its elastic collision with water molecules. These recoiled protons ionize the water molecules along their paths. Due to the short range and high linear energy transfer (LET) of heavy charge particles, the recoiled protons leave a track with a large number of free radicals in a small volume. In about $1 \mu \mathrm{s}$, four species $-\mathrm{H}_{3} \mathrm{O}^{+}$, $\mathrm{OH}, \mathrm{e}_{\mathrm{aq}}^{-}, \mathrm{H}$ and $\mathrm{H}_{2}$, are formed. These species diffuse and subsequently react with each other and some will recombine. These free radicals will react with biological molecules and may cause the death of these cells.[46]

There are approximately 3400 treated patients and 1500 in the United States.[47] Researches conducted in the Gershenson Radiation Oncology Center of Wayne State University (WSU) and Maruyama have shown that neutron brachytherapy is a more effective way to deal with bulky tumors, hypoxic tumors and recurrent tumors when compared with photon therapy.[48] These bulky tumors include gynecological tumors, head and neck, rectal, sarcomas, etc. Currently, the Gershenson Radiation Oncology Center of WSU is the only medical institute in the United States treating cancer patients with neutron brachytherapy. 


\subsubsection{Radioisotope Neutron Sources}

As mentioned in the previous section, ${ }^{252} \mathrm{Cf}$ can be used as a neutron source for brachytherapy. Californium-252 has a half-life of 2.645 years. It decays by alpha emission (96.9\%) and spontaneous fission (3.1\%). It emits 3.768 neutrons per fission on average. It has a neutron energy spectrum that may be fit to a Watt fission model with a most probable neutron energy of $0.7 \mathrm{MeV}$.[49] The average energy is approximately 2.3 MeV. One milligram of ${ }^{252} \mathrm{Cf}$ emits $2.3 \times 10^{9} \mathrm{n} / \mathrm{s}$. The fabrication process for the ${ }^{252} \mathrm{Cf}$ seeds are relatively more difficult than most gamma sources due to potential pressurization via its alpha decay branch.[47] The source used by the clinicians at WSU contains $\leq 30 \mu \mathrm{g}{ }^{252} \mathrm{Cf}$ in the form of a cermet wire of $\mathrm{Cf}_{2} \mathrm{O}_{3}$ in a palladium matrix. The treatment time for each patient can be as long as several hours. Furthermore, the source has to be manually loaded into the patients with this Low-Dose-Rate (LDR) design. The clinicians inevitably handle a radioactive source routinely. Due to these drawbacks, remotely afterloaded sources have been designed in the United States and a ${ }^{252} \mathrm{Cf}$ loading of $100 \mu \mathrm{g}$ have been reported.[47] High-Dose-Rate (HDR) design had been deployed in the European countries. The schematic diagram of a HDR system is shown in Figure 7.2.1.

\subsubsection{Fusion-based Neutron sources}

A fusion-based neutron source for brachytherapy offers several advantages over the ${ }^{252} \mathrm{Cf}$ brachytherapy neutron source in terms of safety concerns. A D-D/D-T neutron brachytherapy neutron generator produces no radioactivity when the neutron generator is not in operation. Using an accelerator based neutron source complies with the ALARA 


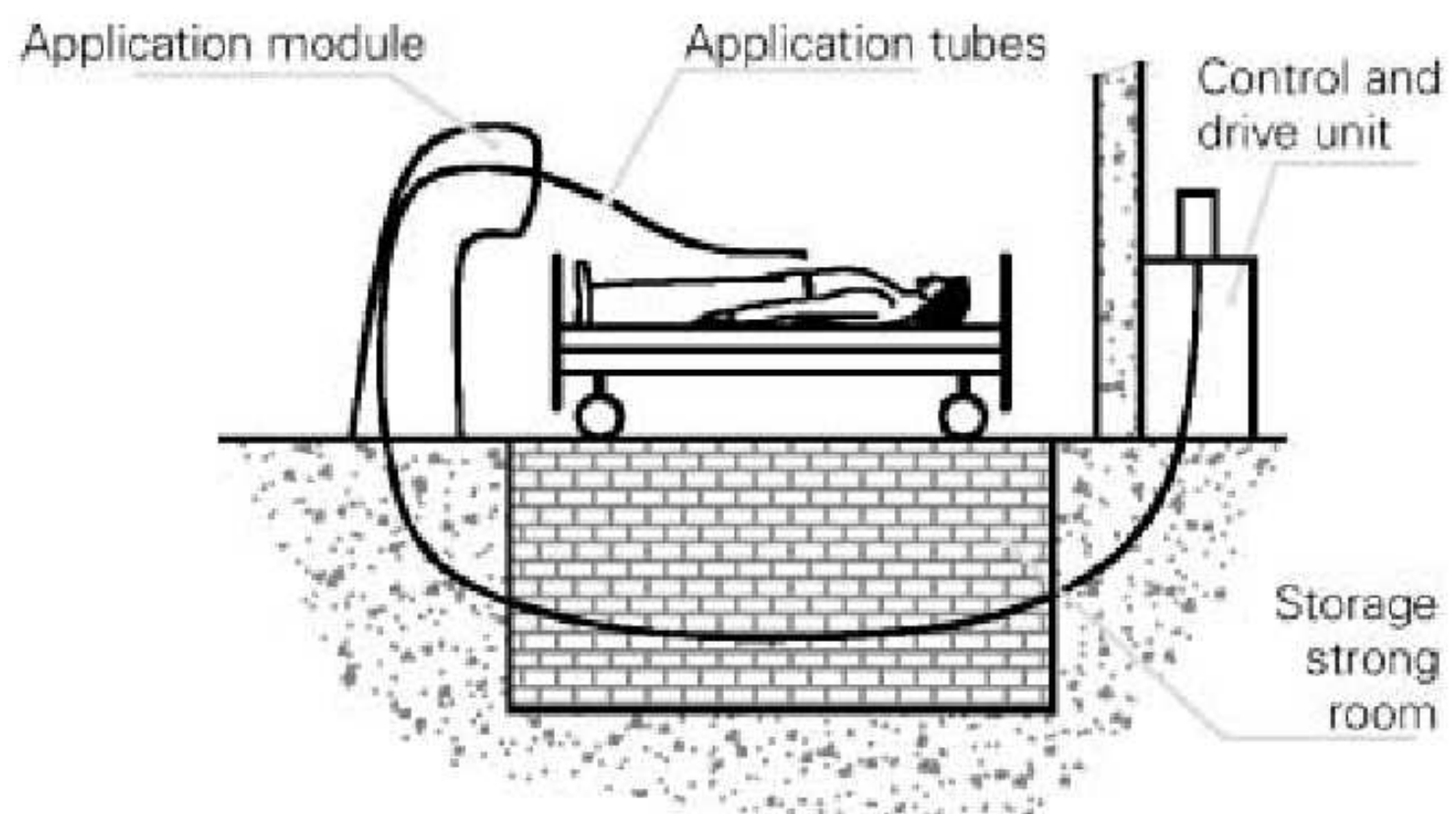

Figure 7.2.1 A HDR ${ }^{252} \mathrm{Cf}$ neutron brachytherapy treatment system

(As Low As Reasonably Achievable) policy set forth by the U.S. Nuclear Regulatory (NRC). Second, with the current LDR ${ }^{252} \mathrm{Cf}$ system, the treatment time for each patient is several hours. A $100 \mathrm{keV}$ D-D neutron generator operating at $1 \mathrm{~mA}$ is expected to produce $10^{9} \mathrm{n} / \mathrm{s}$. Assuming that the RBE of $2.5 \mathrm{MeV}$ neutron is approximately equal to that of ${ }^{252} \mathrm{Cf}$ spontaneous fission neutrons, a $0.1 \mathrm{~mA} \mathrm{D}-\mathrm{D}$ neutron generator is capable of treating more than one patient per hour.

The fusion-based neutron brachytherapy system at LBNL is based on the focused ion beam technology developed for lithography. Beam spot size can be focused down to less than $1 \mu \mathrm{m}$.[50] The $\mathrm{D}^{+}$ion beam can be focused down to $1 \mathrm{~mm}$ in diameter or less. The titanium coated conical target has a diameter of $\sim 3 \mathrm{~mm}$ for target cooling reason. The surface area of the target is thus larger than $0.07 \mathrm{~cm}^{2}$. The total power of the beam is $(0.1 \mathrm{~mA} \times 100 \mathrm{kV})=10 \mathrm{~W}$. An average power density of $<143 \mathrm{~W} / \mathrm{cm}^{2}$ is obtained at the 
target. This heat rate can be dissipated by a moderate water flow rate of $1.45 \mathrm{~L} / \mathrm{min}$ at 2 atm.[51] Figure 7.2.2 shows a model of the D-D fusion-based neutron generator. Several of these neutron generators can be mounted on a single station as shown in Figure 7.2.3 to treat multiple tumor sites at the same time.

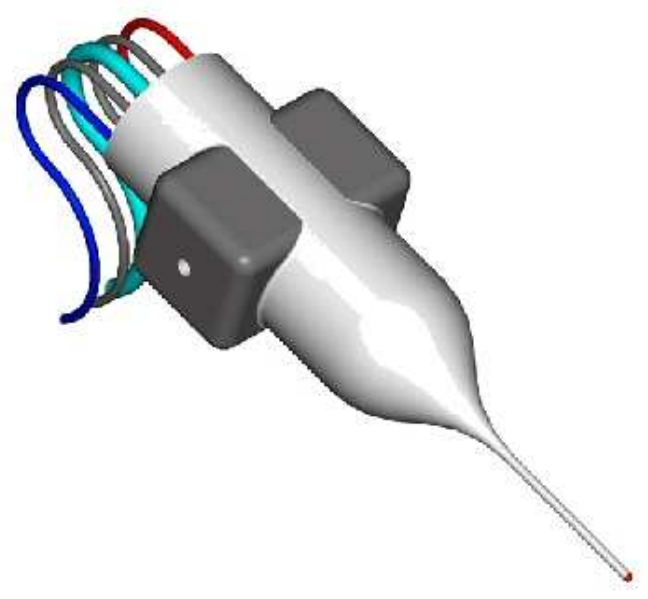

Figure 7.2.2 A fusion-based neutron generator for brachytherapy

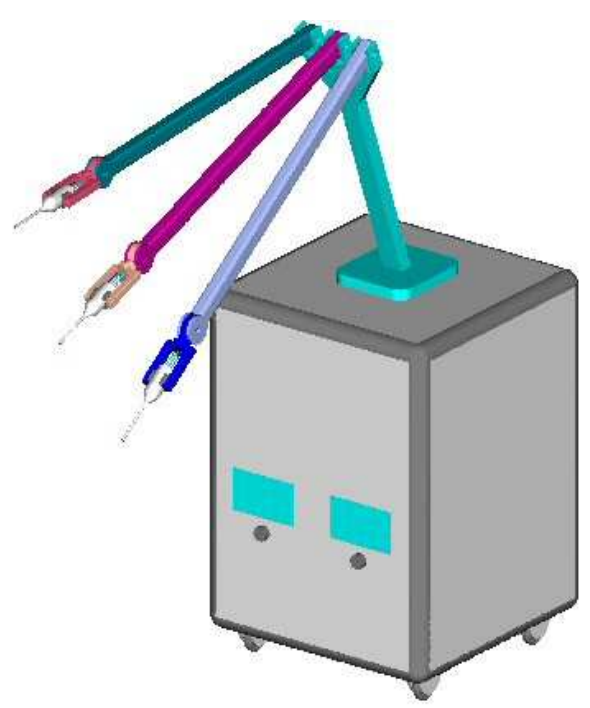

Figure 7.2.3 Multiple neutron generators mounted on a single station

\subsubsection{Neutron dose profile, RBE and activation rates}

The neutron dose profile due to a D-D brachytherapy needle is plotted as a function of distance from the source in Figure 7.2.4. The exact treatment time for a $10^{8}$ D-D n/s point source cannot be calculated without knowing the exact neutron RBE for the tumors being dealt with. The RBE for neutron can be calculated if the RBE's for the neutron induced charge particles is determined experimentally. Table 7.2.1 lists all the neutron induced charge particle production reactions with their threshold energy and products. Among those reactions, only ${ }^{14} \mathrm{~N}(\mathrm{n}, \mathrm{p})$ has a significant cross-section for energy below $2.45 \mathrm{MeV}$. 


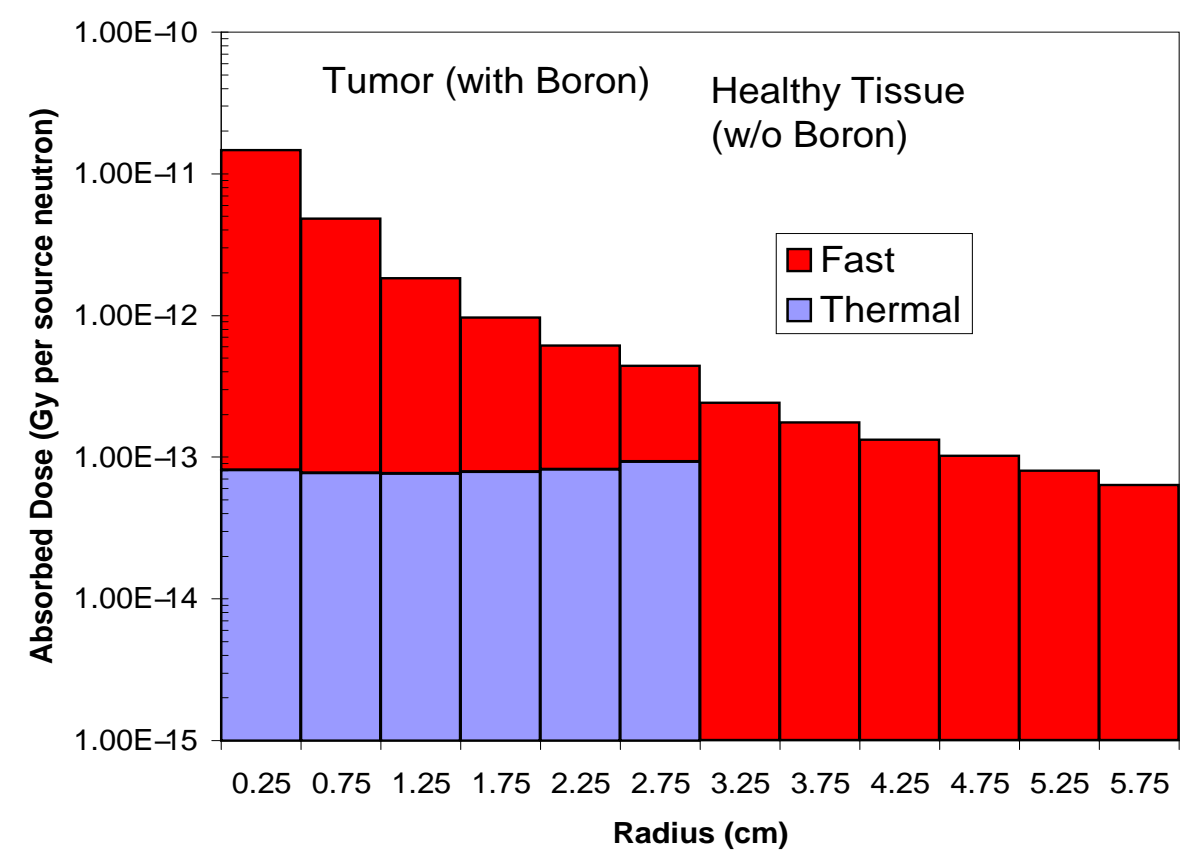

Figure 7.2.4 Dose Profile due to a point D-D neutron source

\begin{tabular}{|l|l|l|l|}
\hline Element & $\begin{array}{l}\text { Neutron induced } \\
\text { reaction }\end{array}$ & $\begin{array}{l}\text { Threshold energy } \\
(\mathrm{MeV})\end{array}$ & Product \\
\hline${ }^{16} \mathrm{O}$ & $(\mathrm{n}, \alpha)$ & 2.35533 & ${ }^{13} \mathrm{C}$, stable \\
\hline${ }^{16} \mathrm{O}$ & $(\mathrm{n}, \mathrm{p})$ & 10.24595 & ${ }^{16} \mathrm{~N}, \mathrm{~T}_{1 / 2}=7.13 \mathrm{~s}$ \\
\hline${ }^{16} \mathrm{O}$ & $(\mathrm{n}, \mathrm{d})$ & 10.52741 & ${ }^{15} \mathrm{~N}$, stable \\
\hline${ }^{12} \mathrm{C}$ & $(\mathrm{n}, \alpha)$ & 6.18044 & ${ }^{9} \mathrm{Be}$, stable \\
\hline${ }^{12} \mathrm{C}$ & $(\mathrm{n}, 3 \alpha)$ & 7.88628 & $\mathrm{n}$ \\
\hline${ }^{12} \mathrm{C}$ & $(\mathrm{n}, \mathrm{n})$ & 7.98584 & ${ }^{8} \mathrm{Be}$, stable \\
\hline${ }^{12} \mathrm{C}$ & $(\mathrm{n}, 2 \alpha)$ & 8.85109 & ${ }^{5} \mathrm{He}$, stable \\
\hline${ }^{12} \mathrm{C}$ & $(\mathrm{n}, \mathrm{p})$ & 13.64462 & ${ }^{12} \mathrm{~B}, \mathrm{~T}_{1 / 2}=20.20 \mathrm{~ms}$ \\
\hline${ }^{14} \mathrm{~N}$ & $(\mathrm{n}, \mathrm{p})$ & 0 & ${ }^{14} \mathrm{C}, \mathrm{T}_{1 / 2}=5700 \mathrm{yrs}$ \\
\hline${ }^{14} \mathrm{~N}$ & $(\mathrm{n}, \alpha)$ & 0.16955 & ${ }^{11} \mathrm{~B}$, stable \\
\hline${ }^{14} \mathrm{~N}$ & $(\mathrm{n}, 2 \alpha)$ & 9.45835 & ${ }^{7} \mathrm{Li}$, stable \\
\hline${ }^{14} \mathrm{~N}$ & $(\mathrm{n}, \mathrm{d})$ & 5.70968 & ${ }^{13} \mathrm{C}$, stable \\
\hline${ }^{14} \mathrm{~N}$ & $(\mathrm{n}, \mathrm{t})$ & 4.30430 & ${ }^{12} \mathrm{C}$, stable \\
\hline${ }^{14} \mathrm{~N}$ & $(\mathrm{n}, 2 \mathrm{n})$ & 11.31363 & ${ }^{13} \mathrm{~N}, \mathrm{~T}_{1 / 2}=9.965 \mathrm{minutes}$ \\
\hline
\end{tabular}

Table 7.2.1 Neutron induced charge particles in major tissue elements 
Activation of these major elements in generic tissues is not a concern. The two major cell killing mechanisms, as mentioned previously, are the recoiled protons and the protons produced in ${ }^{14} \mathrm{~N}(\mathrm{n}, \mathrm{p})$ reaction. The neutron $\mathrm{RBE}$ can be studied with low energy proton beam uniformly over an irradiation area using radiochromic film.

\subsubsection{Summary}

There are many approaches that can detect a small amount of explosives in a luggage with acceptable probability of detection and false alarm rate. (e.g. API and NRR) Unfortunately, the cost, screening rate, and physical weight of these systems have kept people from adopting these technologies in airport. By combining the X-ray images with TNA, the hybrid system may be able to provide both high quality images of luggages being interrogated with material identification capability at low cost. A lot of research efforts are needed in order to make such a system to work flawlessly. The development of an intellectual algorithm of unfolding the signals obtained from the detectors is the essential part to make this approach working with satisfaction. The design of such an algorithm is beyond this scoping study.

Fast neutron brachytherapy has the advantage of high RBE for certain tumors with high photon radioresistivity. If the neutron source is a radioisotope source, the source must be stored and shielded to avoid accidental exposure. Clinical personnel prefer to receive a minimal dose from the neutron sources. The D-D neutron generator for brachytherapy would be an ideal solution to avoid occupational dose. However, there are several issues that have to be addressed. The RBE's for fast neutron $(<2.45 \mathrm{MeV})$ for different tumors need to be studied well enough to justify the high morbidity of using neutron brachytherapy rather than photon brachytherapy. The size of the D-D neutron 
generator for brachytherapy may be too large or its applicator is too rigid to reach some tumors adjacent to inner organs. 


\section{Chapter 8}

\section{Conclusion}

This study has analyzed various neutron sources and their applications, with emphasis on the new fusion-based neutron generator designs. Two types of the new D-D neutron generators were described in detail: the axial and coaxial types. The neutron yield for the axial neutron generator is $>10^{8} \mathrm{n} / \mathrm{s}$ while the neutron yield for coaxial for the coaxial neutron generator is $>10^{9} \mathrm{n} / \mathrm{s}$. The concept of low energy high current fusionbased neutron generators has been demonstrated to work as expected. The prototypes being tested at LBNL will continue to be upgraded in order to achieve the envisioned yield of $10^{12} \mathrm{D}-\mathrm{D} \mathrm{n} / \mathrm{s}$ and $10^{14} \mathrm{D}-\mathrm{T} \mathrm{n} / \mathrm{s}$. The neutron yield is not the only parameter that determines the usefulness of a neutron source. The source neutron density or source neutron brightness is one of the major reasons that these fusion-based neutron generators can provide a flux comparable to other neutron sources with orders of magnitude higher neutron yield.

Regarding the NAA experiment performed in the prototype neutron facility, we were able to identify the bulk elements in the sample. With further improvements in the 
prototype and increase in the neutron yield, we will be able to identify the trace element as well.

The Monte Carlo code MCNP was used for all computer simulations. The detailed analysis of the variance reduction techniques used to speedup the calculation and increase accuracy, was included.

Regarding thermal neutron scattering, a D-T fusion-based neutron source is predicted to produce a thermal neutron flux of $7.3 \times 10^{5} \mathrm{n} / \mathrm{cm}^{2} \mathrm{~s}$ at the sample position. The resolution for thermal neutrons is less than $5 \%$ while the resolution of cold neutrons is less than $2 \%$. Therefore, the D-T neutron generator is more suitable for cold neutron scattering because of its long ion beam pulse. Cold neutron can also take advantages of supermirror and the spin-echo small angle neutron scattering technique. This neutron generator could be efficiently used for research and educational purposes at universities.

The concepts of using fission multiplier have been studied carefully in this thesis. The main disadvantage of deploying such a system could be its fissile material loading that, for some designs, could be two to ten times that of a small-scale research reactor. In addition, the sample being irradiated has to see an optically thin fuel assembly in order to maximize the gain. Also, the fuel with high fissile contents is needed in order to minimize the size of the subcritical pile. A gain of 25 was predicted for positron production while a gain of 17 was predicted for BNCT. Therefore, it could be more practical to use the D-T reaction to increase neutron yield rather than coupling a D-D generator with a fission multiplier. The amount of tritium in a D-T neutron generator could be minimized, by employing existing techniques. Any neutron generator containing either tritium or fissile material must be designed with safety, security and non- 
proliferation issues in mind. The author believes that the proper regulations and licensing procedures will make a D-T neutron generator more favorable than a D-D driven subcritical fission multiplier neutron source.

As for neutron generators for brachytherapy, detailed RBE studies must be carried out in order to ensure that the D-D neutrons have clinical advantages for the tumors being treated. Perhaps, a prescription of fast neutron therapy can be based upon the relative status of appropriate molecular parameters that show a differential impact upon radiosensitivity.

A number of applications of the fusion-based neutron generators have been identified to be viable: BNCT, neutron scattering, NAA, and neutron brachytherapy, if the envisioned neutron yield is achieved. The neutron yield and neutron brightness have to be brought up to the required level obtained in computer simulations. Continuous studies and the experimental findings in axial electron confining magnetic field, low power CW RF discharge, and optimum Ti target temperature will enable us to improve the neutron yield toward to the desired values. 


\section{Bibliography}

[1] J. Csikai, Handbook of Fast Neutron Generators, CRC Press, Inc., Boca Raton, Florida, USA, (1987)

[2] P. Antonzzi, "Scattering Effects in Fusion Plasma Neutron Diagnostics", Uppsala University, Sweden, (1995)

[3] "NRL Plasma Formulary", 44-5, (2002)

[4] T. Beas, "Nuclear Research Reactors in the World", (1999), $<$ http://www.iaea.org/worldatom/rrdb>

[5] D. Richter and T. Springer, "A Twenty Years Forward Look at Neutron Scattering Facilities in the OECD Countries and Russia", 18, (1998)

[6] E.I. Litvinenko, "IBR-2", (2000), <http://nfdfn.jinr.ru/userguide-97/ibr-2>

[7] K.J. Riley, "Construction and Characterization of a Fission Converter Based Epithermal Neutron Beam for BNCT", Massachusetts Institute of Technology, (2001)

[8] I.I. Degtyarev, O.A. Liashenko, I.A. Yazynin, V.I. Belyakov-Bodin, et al, "Calculational Estimations of Neutron Yield from ADS Target", Proceedings of the 2001 Particle Accelerator Conference, Chicago, 2796-8, (2001)

[9] J.B. Donahue, T.O. Brun, P.D. Ferguson, et al, "New Spallation Target and 
Moderator System at the Lujan Center", LANSCE Activity Report 1995-1998, $138-40,(1998)$

[10] Y. Saji, "Energy Spectrum and Angular Distributions of Neutrons from the Reaction ${ }^{9} \mathrm{Be}(\mathrm{p}, \mathrm{n})^{9} \mathrm{~B}$ at 8 to $14 \mathrm{MeV}$ of Proton Energies", Journal of the Physical Society of Japan, 15, 3, 367-71, (1960)

[11] J.W. Meadows, "The ${ }^{9} \mathrm{Be}(\mathrm{d}, \mathrm{n})$ Thick-Target Neutron Spectra for Deuteron Energies Between 2.6 and 7.0 MeV", Nuclear Instruments and Methods in Physics Research $\underline{\mathrm{A}}, \mathbf{3 2 4}, 239-46,(1993)$

[12] L.T. Perkins, G.J. De Vries, P.R. Herz, W.B. Kunkel, K.N. Leung, et al, "Performance Characterization of RF-Driven Multicusp Ion Sources", Rev. Sci. Instrum, 67, 1057, (1996)

[13] J. Reijonen, G. English, et al, "Compact Neutron Generator Development at LBNL", (to be published)

[14] J.F. Briesmeister, "MCNP -- A General Monte Carlo N-Particle Transport Code", LA-12625-M, Los Alamos National Laboratory, (2000)

[15] T.E. Booth, "A Sample Problem for Variance Reduction in MCNP", LA-10363MS, Los Alamos National Laboratory, (1985)

[16] C.L. Royer, "Los Alamos Natinoal Laboratory: MCNP Resources for Users", (2001), <http://laws.lanl.gov/x5/MCNP/theresources.html>

[17] G.L. Squires, Introduction to the Theory of Thermal Neutron Scattering, Cambridge Univ. Press, (1978)

[18] K. Bennett, R.V. Dreele, H.-R. Wenk, "HIPPO (High Pressure Preferred Orientation) Research Applications", 33, (1998) 
[19] X.-L. Wang, “Conceptual Design of the SNS Engineering Diffractometer”, 12, (2000)

[20] R. Pynn, "Neutron Scattering: A Primer", Los Alamos Science, (1990)

[21] C. Hugenschmidt, G. Kogel, R. Repper, K. Schreckenback, et al, "Monoenergetic Positron Beam at the Reactor Based Positron Source at FRM-II", Nuclear Instruments and Methods in Physics Research B, 192, 97-101, (2002)

[22] M. Jibaly, A. Weiss, A.R. Koymen, D. Mehl, L. Stiborek, and C. Lei, "Measurement of the Positron Work Functions of the Polycrystalline Fe, Mo, Ni, Pt, Ti, and V", Physical Review B, 44, 12166, (1991)

[23] J. Verbeke, J.L. Vujic, and K.-N. Leung, "Neutron Beam Optimization for Boron Neutron Capture Therapy Using the D-D and D-T High-Energy Neutron Source", Nuclear Technology, 129, 257, (2000)

[24] O.K. Harling, K.J. Riley, T.H. Newton, B.A. Wilson, et al, “The New Fission Converter Based Epithermal Neutron Irradiation Facility at MIT”, Ninth International Symposium on Neutron Capture Therapy for Cancer, PL-1, 7, (2000)

[25] E. Greenspan, private conversation, 2003

[26] D.D. Keiser Jr. and M.C. Petri, "Interdiffusion Behavior in U-Pu-Zr Fuel Versus Stainless Steel Couples", Journal of Nuclear Materials, 240, 51-61, (1996)

[27] K.H. Kim, D.B. Lee, C.K. Kim, G.E. Hofman, and K.W. Paik, "Characterization of U-2 wt\% Mo and U-10 wt\% Mo Alloy Powders Prepared by Centrifugal Atomization", Nuclear Engineering and Design, 245, 179-84, (1997)

[28] K.H. Kim, J.M. Park, C.K. Kim, G.L. Hofman, and M.K. Meyer, "Irradiation Behavior of Atomized U-10 wt.\% Mo Alloy Aluminum Matrix Dispersion Fuel 
Meat at Low Temperature", Nuclear Engineering and Design, 211, 229-35, (2002)

[29] T. Gozani, P. Ryge and P. Shea, "Explosive Detection System Based on Thermal Neutron Activation", IEEE AES Magazine, 12, 17-20, (1989)

[30] W.C. Lee, D.B. Mahood, P. Ryge, P. Shea, T. Gozani, "Thermal Neutron Analysis (TNA) Explosive Detection Based on Electronic Neutron Generators", Nuclear Instruments and Methods in Physics Research B, 99, 739-742, (1995)

[31] T. Gozani, M. Elsalim, D. Strellis, D.R. Brwon, D.B. Mahood, and P. Ryge, "SEAVEDS - Nonintrusive Inspection of Maritime Vessels for Concealed Drugs"

[32] B. Maglich, C.W. Powell, et al, "Demo of Chemically-Specific Non-Intrusive Detection of Cocaine Simulant by Fast Neutron Atometry", ONDCP International Technology Symposium, (1999), <http://www.hienergyinc.com/products/SuperSenzor_tech.htm>

[33] J.C. Overley, "Element-Sensitive Computed Tomography With Fast Neutrons", Nuclear Instruments and Methods in Physics Research B, 24/25, 1058-1062, (1987)

[34] J.C. Overley, "Explosives Detection Through Fast-Neutron Time-of-Flight Attenuation Measurements", Nuclear Instruments and Methods in Physics Research $\underline{\mathrm{B}}, \mathbf{9 9}, 728-732,(1995)$

[35] "The Practicality of Pulsed Fast Neutron Transmission Spectroscopy For Aviation Security", Tensor Technology, (1999)

[36] G. Chen, and R.C. Lanza, "Fast Neutron Resonance Radiography For Elemental Mapping"

[37] J.P.O. Evans, "Stereoscopic Imaging Using Folded Linear Dual-Energy X-ray Detectors", Measurement Science And Technology, 13, 1388-1397, (2002) 
[38] W. Lee, J. Bendahan, P. Shea, V. Leung, "Technical updates from the TNA operations program", Nuclear Instruments \& Methods in Physics Research A, 641-645, (1994)

[39] L. Helmuth, "Seeing Through Steel", (1999), <http://www.inel.gov/featurestories/8-99pins.shtml>

[40] B. Hasegawa, H.R. Tang, A.J. Da Silva, K.H. Wong, K. Iwata, M.C. Wu, "DualModality Imaging", Nuclear Instruments and Methods in Physics Research A, 471, 14-144, (2001)

[41] M.C. Joiner, "Basic Clinical Radiobiology", Particle Beams in Radiotherapy, 173-183, (1997)

[42] R.A. Britten, L.J. Peters, and D. Murray, "Biological Factors Influencing the RBE of Neutrons: Implications for Their Past, Present and Future Use in Radiotherapy",

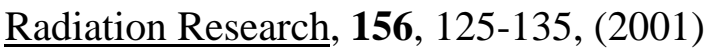

[43] C.S. Wuu, P. Kliauga, M. Zaider, and H.I. Amols, "Microdosimetric Evaluation of Relative Biological Effectiveness for 103Pd, 125I, 241Am and 192Ir Brachytherapy Sources", Int. J. Radiation Oncology Biol. Phys., 36, 3, 689-697, (1996)

[44] R.C. Martin and D.S. Halpern, "Development of Miniature High-Dose-Rate Cf-252 Sources for Boron Enhanced and Fast Neutron Brachytherapy", Proc. 9th ISNCT, (2000)

[45] Y. Maruyama, J.M. Feola, J. Wierzbicki, J.R. van Nagell, et al, "Clinical study of relative biological effectiveness for cervical carcinoma treated by $252 \mathrm{Cf}$ neutrons and assessed by histological tumor eradication", British Journal of Radiology, 
63,748, 270-277, (1990)

[46] R.E. Faw, and J.K. Shultis, "Biological Effects of Ionizing Radiation",

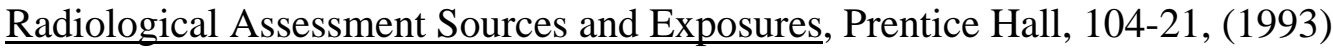

[47] R.C. Martin, "Development of High-Dose-Rate Californium-252 Sources for Neutron Brachytherapy", (2002), <http://www.ornl.gov/divisions/nuclear_science_technology/cuf/cf252b.htm>

[48] R.C. Martin, R.R. Laxson, J.H. Miller, J.G. Wierzbicki, and et al, "Development of High-activity Cf-252 Sources for Neutron Brachytherapy", Appl. Radiat. Isot., 48, 10-12, 1567-1570, (1997)

[49] M.J. Rivard, J.G. Wierzbicki, and F. Van de Heuvel, "Clinical Brachytherapy with Neutron Emitting 252Cf Sources and Adherence to AAPM TG-43 Dosimetry Protocol", Medical Physics, 26, 1, 87-96, (1999)

[50] Y. Lee, Q. Ji, K.N. Leung, and N. Zahir, "Nanobeam Production With the Multicusp Ion Source", Review of Scientific Instruments, 71, 2, 722-4, (2000)

[51] S. White, "Interstitial/Intracavity Neutron Probe, (1999), <http://web.mit.edu/laba/www/probe.html> 


\section{Appendix}

\section{A. Input files for MCNP simulations}

\section{A.1 Building 52 - the prototype neutron facility}

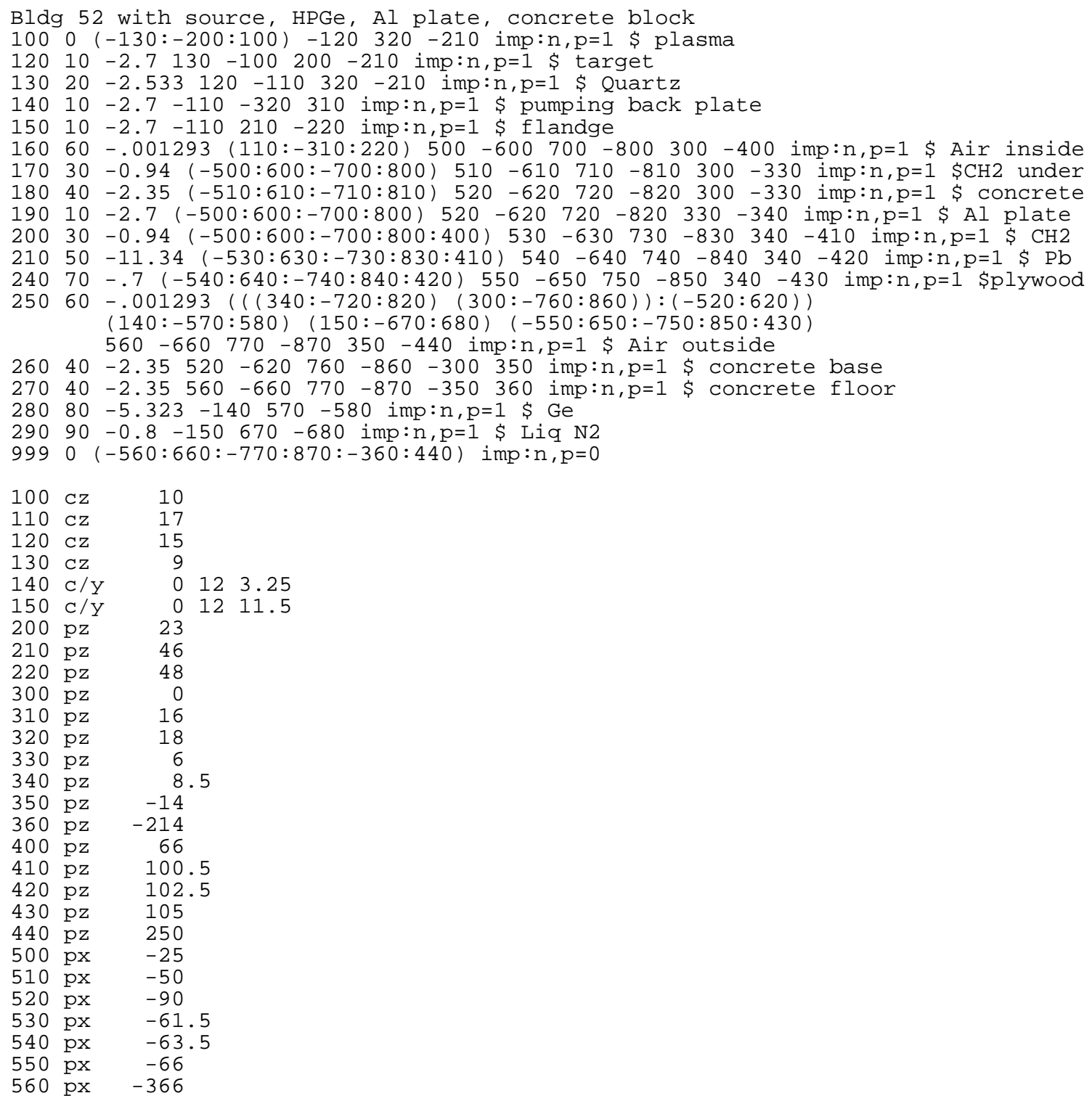




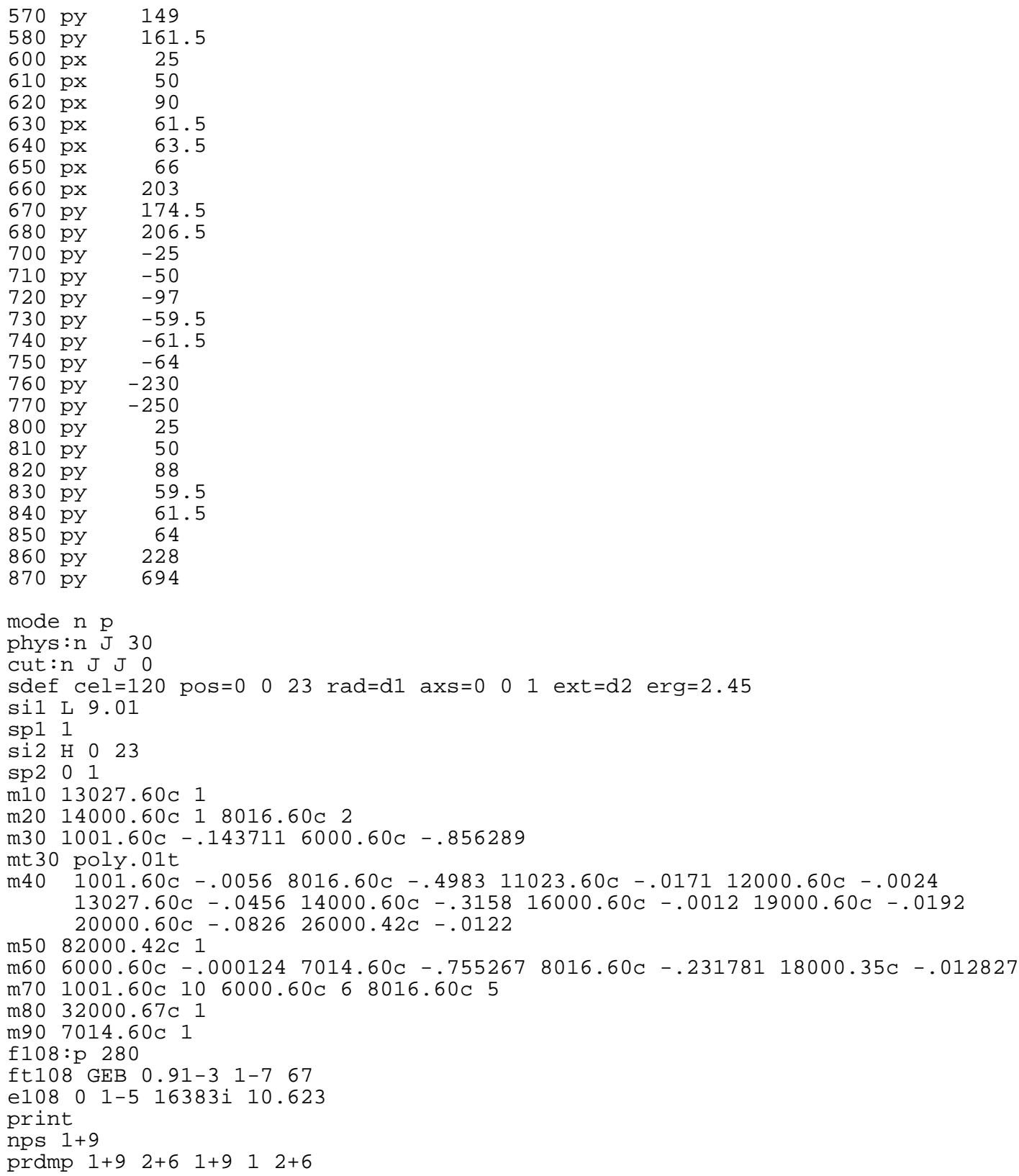

\section{A.2 Neutron scattering at $2 \theta=20^{\circ}$}

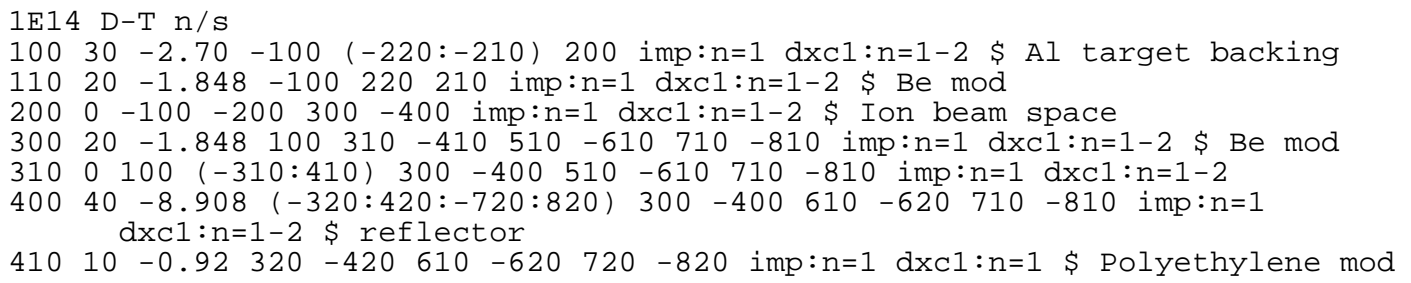




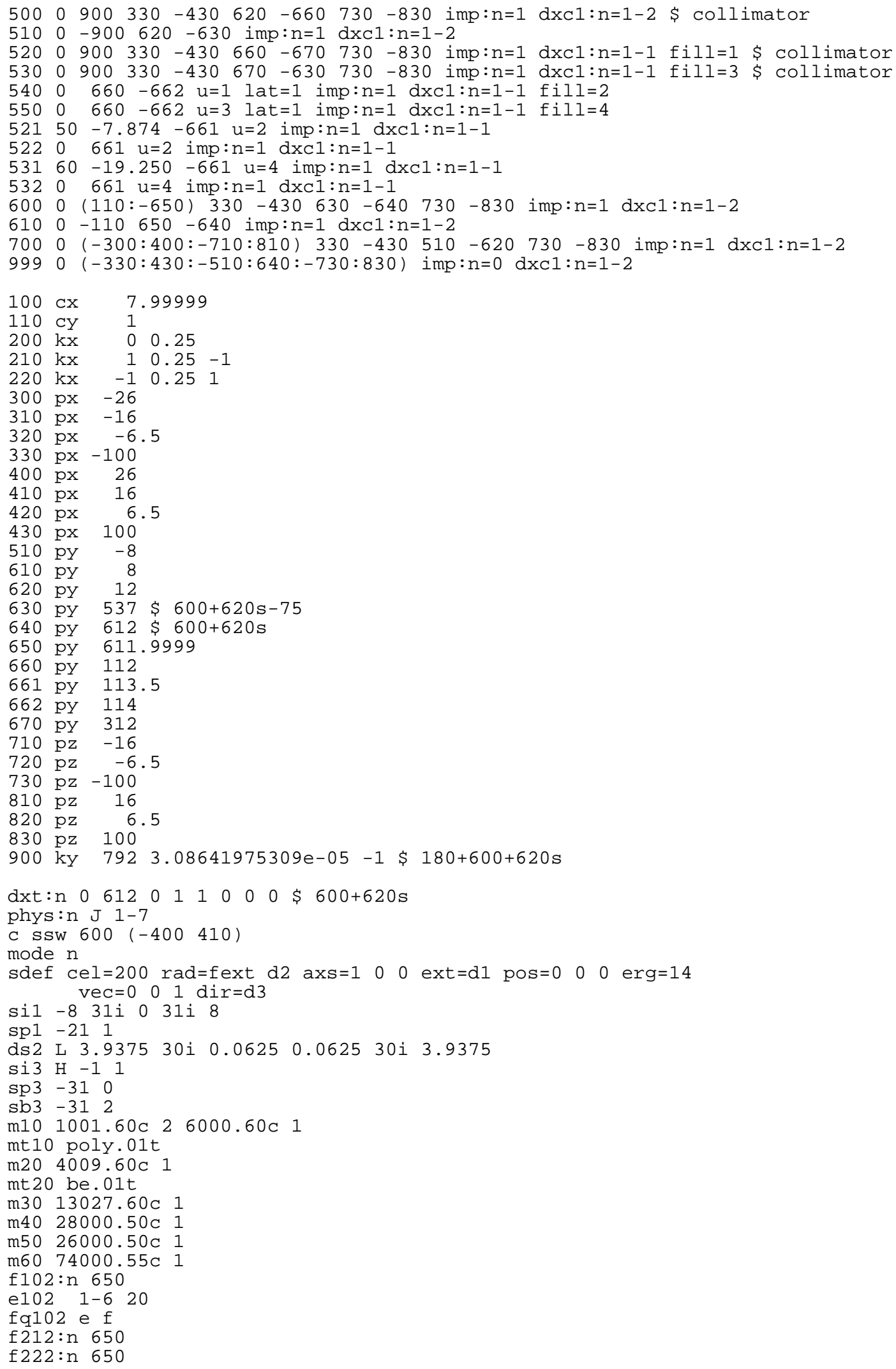




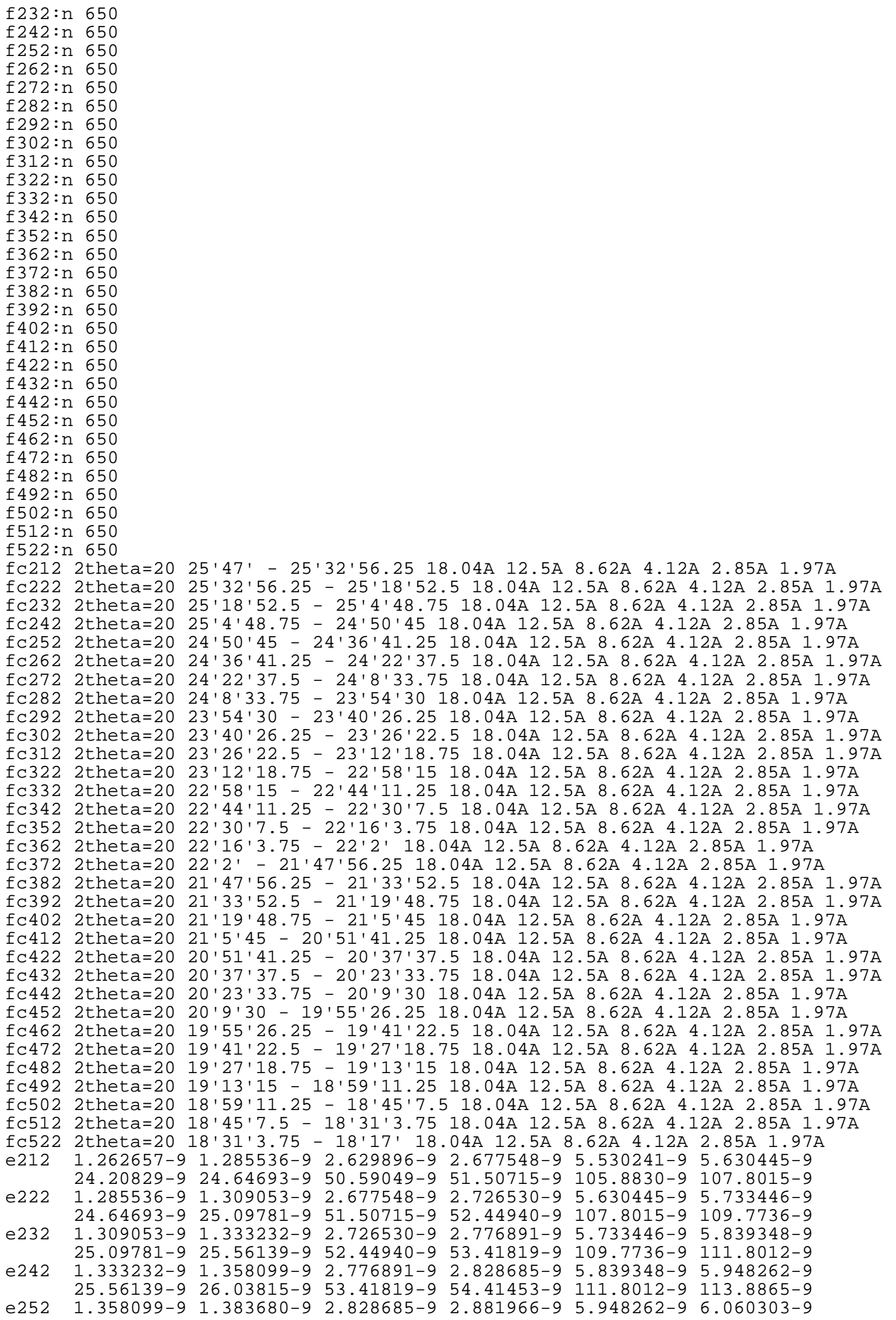


$26.03815-9 \quad 26.52861-9 \quad 54.41453-9 \quad 55.43948-9 \quad 113.8865-9 \quad 116.0316-9$ e262 1.383680-9 $1.410003-9 \quad 2.881966-9$ 2.936791-9 $6.060303-96.175592-9$

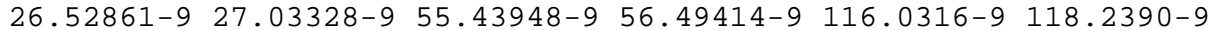

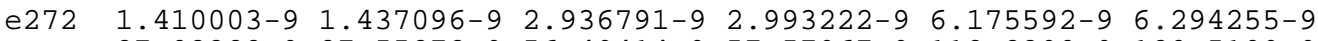

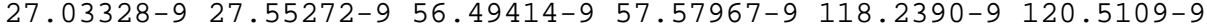

e282 1.437096-9 $1.464990-9 \quad 2.993222-9 \quad 3.051320-9 \quad 6.294255-9 \quad 6.416426-9$

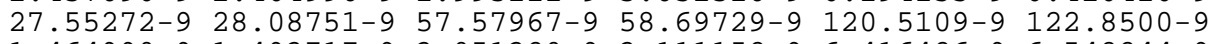

e292 1.464990-9 $1.493717-9 \quad 3.051320-9 \quad 3.111152-9 \quad 6.416426-9 \quad 6.542244-9$

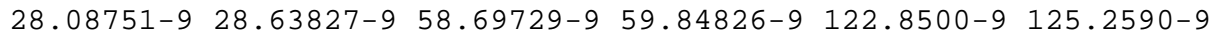

e302 1.493717-9 $1.523309-9 \quad 3.111152-9 \quad 3.172789-9 \quad 6.542244-96.671856-9$

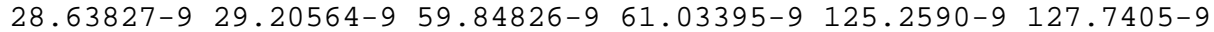

e312 1.523309-9 $1.553803-9 \quad 3.172789-9 \quad 3.236303-9 \quad 6.671856-96.805415-9$

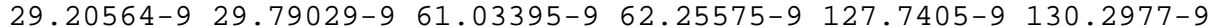

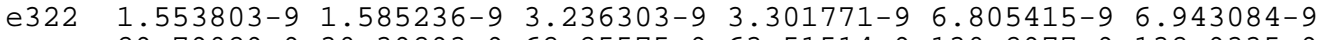

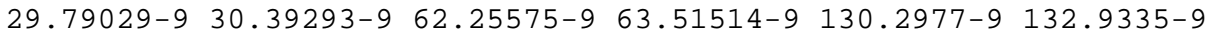

e332 1.585236-9 $1.617645-9 \quad 3.301771-9 \quad 3.369274-9 \quad 6.943084-9 \quad 7.085033-9$

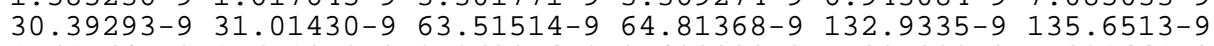

e342 1.617645-9 $1.651073-9 \quad 3.369274-9 \quad 3.438899-9 \quad 7.085033-9 \quad 7.231441-9$

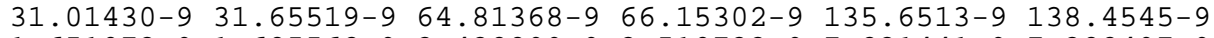

e352 $1.651073-91.685562-9 \quad 3.438899-9 \quad 3.510733-9 \quad 7.231441-97.382497-9$

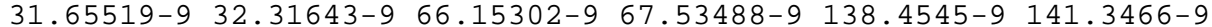

e362 1.685562-9 $1.721158-9 \quad 3.510733-9 \quad 3.584872-9 \quad 7.382497-9 \quad 7.538400-9$

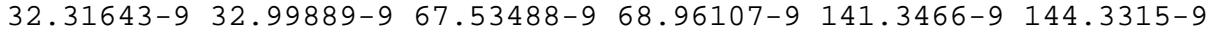

e372 1.721158-9 $1.757908-9 \quad 3.584872-9 \quad 3.661416-9 \quad 7.538400-97.699359-9$

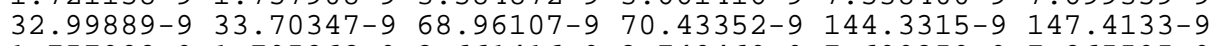

e382 $1.757908-9 \quad 1.795862-9 \quad 3.661416-9 \quad 3.740469-9 \quad 7.699359-9 \quad 7.865595-9$

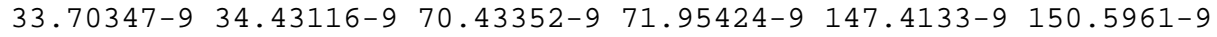

e392 $1.795862-9$ 1.835075-9 $3.740469-9 \quad 3.822143-97.865595-9$ 8.037342-9

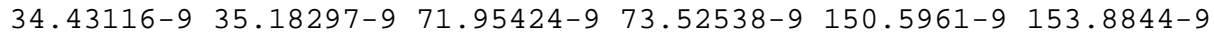

e402 1.835075-9 $1.875603-9 \quad 3.822143-9 \quad 3.906555-9$ 8.037342-9 $8.214846-9$

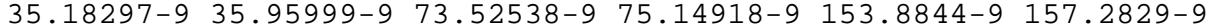

e412 1.875603-9 $1.917504-9 \quad 3.906555-9 \quad 3.993827-9 \quad 8.214846-98.398366-9$

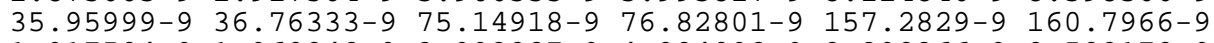

e422 $1.917504-9 \quad 1.960842-9 \quad 3.993827-9 \quad 4.084092-9 \quad 8.398366-9 \quad 8.588178-9$ $\begin{array}{lllllll}36.76333-9 & 37.59423-9 & 76.82801-9 & 78.56441-9 & 160.7966-9 & 164.4308-9\end{array}$

e432 $1.960842-9 \quad 2.005682-9 \quad 4.084092-9 \quad 4.177488-9 \quad 8.588178-9 \quad 8.784573-9$ $37.59423-9 \quad 38.45393-9 \quad 78.56441-9 \quad 80.36103-9 \quad 164.4308-9 \quad 168.1910-9$

e442 2.005682-9 2.052096-9 $4.177488-9 \quad 4.274160-9$ 8.784573-9 8.987859-9

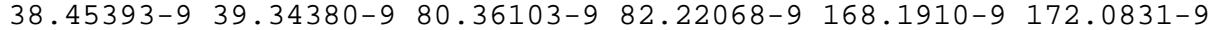

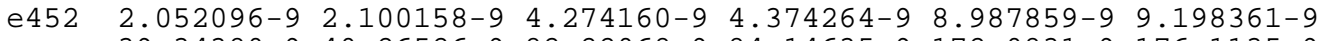
$39.34380-9 \quad 40.26526-9 \quad 82.22068-9 \quad 84.14635-9 \quad 172.0831-9 \quad 176.1135-9$

e462 2.100158-9 $2.149946-9$ 4.374264-9 $4.477964-9$ 9.198361-9 $9.416426-9$ $40.26526-9$ 4i.21983-9 $84.14635-9 \quad 86.14120-9$ 176.1135-9 $180.2886-9$

e472 2.149946-9 $2.201545-9 \quad 4.477964-9 \quad 4.585435-9 \quad 9.416426-9 \quad 9.642420-9$

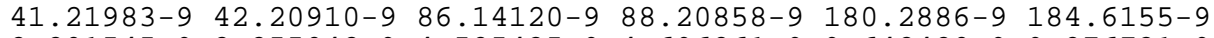

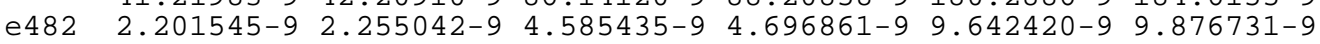

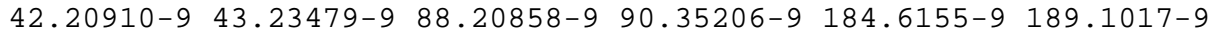

e492 2.255042-9 $2.310534-9 \quad 4.696861-9 \quad 4.812440-9$ 9.876731-9 $10.11977-9$

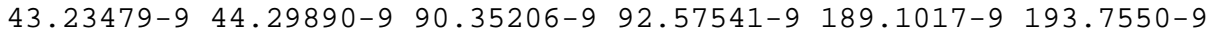

e502 2.310534-9 $2.368119-9 \quad 4.812440-9 \quad 4.932380-9$ 10.11977-9 $10.37199-9$ $44.29890-9 \quad 45.40275-9 \quad 92.57541-9 \quad 94.88266-9$ 193.7550-9 $198.5839-9$

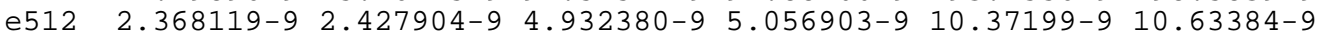

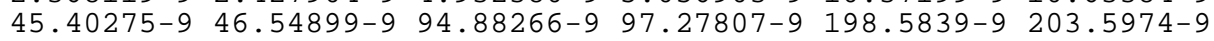

e522 2.427904-9 $2.490004-9 \quad 5.056903-9 \quad 5.186246-9 \quad 10.63384-910.90583-9$ $46.54899-9 \quad 47.73959-9 \quad 97.27807-9 \quad 99.76620-9 \quad 203.5974-9 \quad 208.8049-9$

t212 $12607049 i \quad 14597518238549 i 21118326365849 i 30528955163549 i \quad 638735$ $\begin{array}{lllllll}799935 & 49 i & 926240 & 1154466 & 49 i & 1336750 & 1+37\end{array}$

t222 $12493849 i \quad 14466518074749 i 20928626129149 i 30254754668149 i 632999$ $79275149 i \quad 9179221144098 \quad 49 i \quad 1324745 \quad 1+37$

t232 $12380549 i \quad 14335317910949 i 20738925892249 i 29980454172549 i 627260$ $785564 \quad 49 i 9096001133726 \quad 49 i \quad 13127351+37$

t242 $12267249 i \quad 142041 \quad 17746949 i 205491256552 \quad 49 i 29706053676649 i \quad 621519$ $\begin{array}{lllllll}778373 & 49 i & 901274 & 1123348 & 49 i & 1300719 & 1+37\end{array}$

t252 $12153849 i \quad 14072817582949 i 20359125418149 i 29431553180549 i \quad 615775$ $77118049 i \quad 8929451112966 \quad 49 i \quad 1288698 \quad 1+37$

t262 $12040449 i \quad 13941517418849 i 20169125180949 i 29156852684249 i 610028$ $\begin{array}{llllllll}763983 & 49 i & 884611 & 1102580 & 49 i & 1276671 & 1+37\end{array}$

t272 $11926949 i 138101 \quad 17254649 i 19979124943649 i 28882052187749 i \quad 604279$ $\begin{array}{lllll}756782 & 49 i & 876274 & 1092188 & 49 i \\ 1264639 & 1+37\end{array}$

t282 $11813449 i \quad 136786170904 \quad 49 i \quad 19788924706149 i 28607151691049 i \quad 598527$

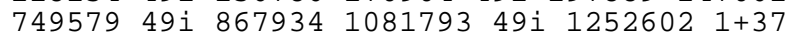


t292 $11699849 i \quad 135471 \quad 169261 \quad 49 i \quad 195986244686 \quad 49 i 28332151194049 i \quad 592773$ $\begin{array}{lllllll}742373 & 49 i & 859589 & 1071392 & 49 i & 1240560 & 1+37\end{array}$

t302 $11586249 i 13415616761749 i \quad 19408324231049 i 28056950696949 i 587016$ $\begin{array}{llllllll}735163 & 49 i & 851242 & 1060988 & 49 i & 1228512 & 1+37\end{array}$

t312 $11472549 i \quad 132839165973 \quad 49 i \quad 192179239933 \quad 49 i \quad 27781750199549 i \quad 581257$ $72795149 i \quad 8428901050578 \quad 49 i \quad 1216459 \quad 1+37$

t322 $11358849 i \quad 13152316432849 i \quad 190274 \quad 237554 \quad 49 i 27506349701949 i \quad 575495$ $72073549 i \quad 834535104016549 i \quad 1204401 \quad 1+37$

t332 $112450 \quad 49 i \quad 130205162682 \quad 49 i \quad 188368235175 \quad 49 i \quad 27230849204149 i \quad 569731$

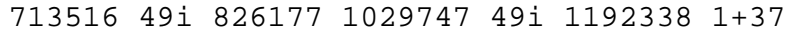

t342 $11131249 i \quad 12888816103549 i \quad 18646223279549 i 26955248706149 i \quad 563965$ $70629549 i \quad 817815 \quad 1019324 \quad 49 i \quad 1180270 \quad 1+37$

t352 $11017349 i \quad 12756915938849 i \quad 18455423041349 i 26679548207949 i \quad 558196$ $69907049 i \quad 8094491008898 \quad 49 i \quad 1168197 \quad 1+37$

t362 $109034 \quad 49 i \quad 126250157740 \quad 49 i \quad 18264622803149 i 26403647709549 i \quad 552425$ $\begin{array}{lllllll}691842 & 49 i & 801081 & 998467 & 49 i & 1156120 & 1+37\end{array}$

t372 $10789549 i \quad 124931 \quad 15609249 i \quad 180738225648 \quad 49 i 26127747210849 i \quad 546652$ $68461249 i \quad 792709988032 \quad 49 i \quad 1144037 \quad 1+37$

t382 $10675549 i \quad 123611 \quad 154442 \quad 49 i \quad 178828 \quad 223264 \quad 49 i 25851646712049 i \quad 540876$

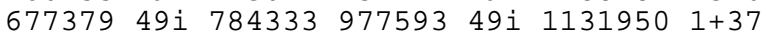

t392 $10561449 i \quad 122290152793 \quad 49 i \quad 176918220879 \quad 49 i 25575546213049 i \quad 535098$ $67014349 i \quad 775955967150 \quad 49 i \quad 1119858 \quad 1+37$

t402 $10447449 i \quad 12096915114249 i \quad 17500721849349 i 25299245713849 i \quad 529318$ $662904 \quad 49 i 767573956703 \quad 49 i \quad 1107761 \quad 1+37$

t412 $10333249 i \quad 11964814949149 i \quad 17309521610649 i 25022845214449 i \quad 523536$ $\begin{array}{llllllll}655662 & 49 i & 759187 & 946251 & 49 i & 1095659 & 1+37\end{array}$

t422 $10219149 i \quad 11832614783949 i \quad 171182 \quad 213718 \quad 49 i 24746344714949 i \quad 517751$ $64841749 i 75079993579649 i \quad 1083553 \quad 1+37$

t432 $101048 \quad 49 i \quad 117003 \quad 146187 \quad 49 i \quad 169269211330 \quad 49 i \quad 24469844215149 i \quad 511964$ $64117049 i 742408925337 \quad 49 i \quad 1071443 \quad 1+37$

t44299906 49i $115680144534 \quad 49 i \quad 167355208940 \quad 49 i 24193143715249 i \quad 506175$ $63392049 i 734013914874 \quad 49 i \quad 1059328 \quad 1+37$

t452 $9876349 i \quad 11435714288049 i \quad 165440206550 \quad 49 i 23916343215049 i \quad 500384$ $62666849 i 725616904407 \quad 49 i \quad 1047208 \quad 1+37$

t462 $9761949 i \quad 11303314122649 i \quad 16352520415849 i 236394 \quad 42714749 i \quad 494591$ $61941349 i 717215893937 \quad 49 i \quad 1035085 \quad 1+37$

t472 $9647649 i \quad 11170913957149 i \quad 161609201766 \quad 49 i 233624 \quad 42214249 i \quad 488796$ $612155 \quad 49 i \quad 708811883462 \quad 49 i \quad 1022956 \quad 1+37$

t482 $9533149 i \quad 11038413791649 i \quad 159692199373 \quad 49 i 23085341713549 i \quad 482999$ $60489549 i 700405872984 \quad 49 i \quad 1010824 \quad 1+37$

t492 $9418749 i 109058 \quad 13626049 i \quad 15777519698049 i 22808241212749 i \quad 477200$ $59763249 i \quad 691995862503 \quad 49 i 998687 \quad 1+37$

t502 $9304249 i \quad 107733134604 \quad 49 i \quad 155857 \quad 19458549 i 22530940711749 i \quad 471399$ $59036749 i \quad 683583852017 \quad 49 i 986546 \quad 1+37$

t512 $9189649 i \quad 10640613294749 i \quad 15393819218949 i 22253540210549 i \quad 465595$ $58309949 i \quad 675167841528 \quad 49 i \quad 974401 \quad 1+37$

t522 $9075149 i \quad 10508013128949 i \quad 152019189793 \quad 49 i \quad 21976139709249 i \quad 459790$ $\begin{array}{lllllll}575829 & 49 i & 666749 & 831036 & 49 i & 962252 & 1+37\end{array}$

ft 212 tmc $0 \quad 5+3$

ft222 tmc $0 \quad 5+3$

ft 232 tmc $0 \quad 5+3$

ft 242 tmc $0 \quad 5+3$

ft 252 tmc $05+3$

ft262 tmc $05+3$

ft272 tmc $05+3$

ft 282 tmc $0 \quad 5+3$

ft 292 tmc $0 \quad 5+3$

ft 302 tmc $0 \quad 5+3$

ft 312 tmc $05+3$

ft 322 tmc $0 \quad 5+3$

ft 332 tmc $0 \quad 5+3$

ft 342 tmc $0 \quad 5+3$

ft 352 tmc $0 \quad 5+3$

ft 362 tmc $0 \quad 5+3$

ft 372 tmc $0 \quad 5+3$

ft 382 tmc $0 \quad 5+3$

ft392 tmc $0 \quad 5+3$

ft 402 tmc $0 \quad 5+3$

ft 412 tmc $0 \quad 5+3$

ft 422 tmc $0 \quad 5+3$

ft 432 tmc $05+3$ 


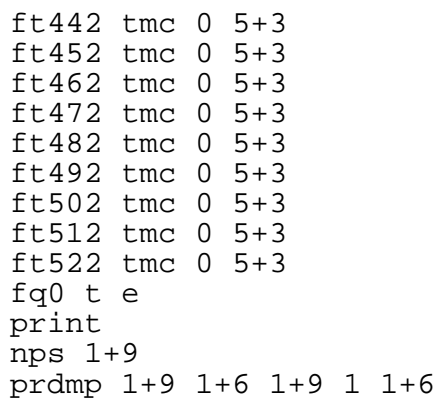

\section{A.3 Neutron scattering at $2 \theta=150^{\circ}$}

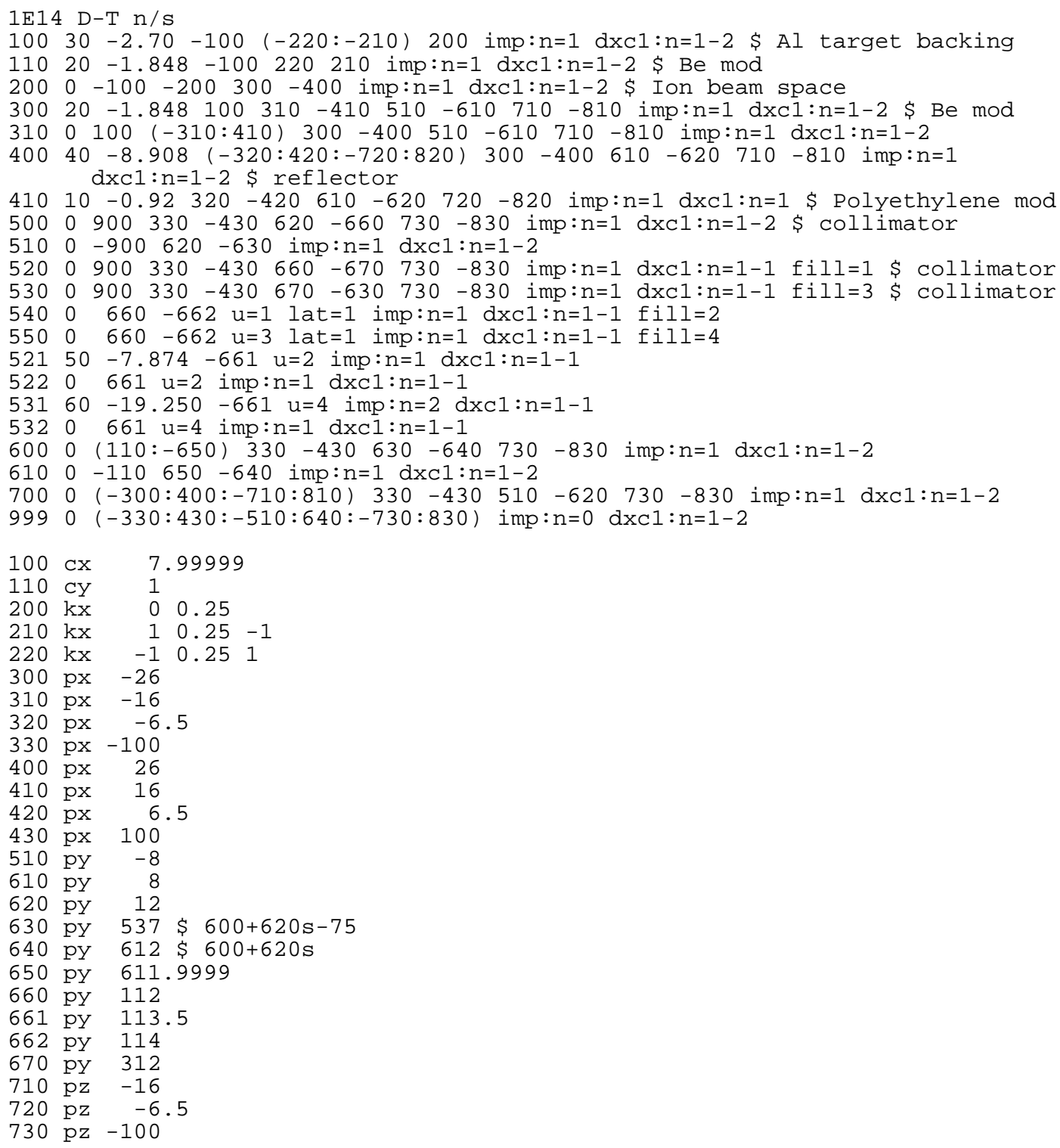




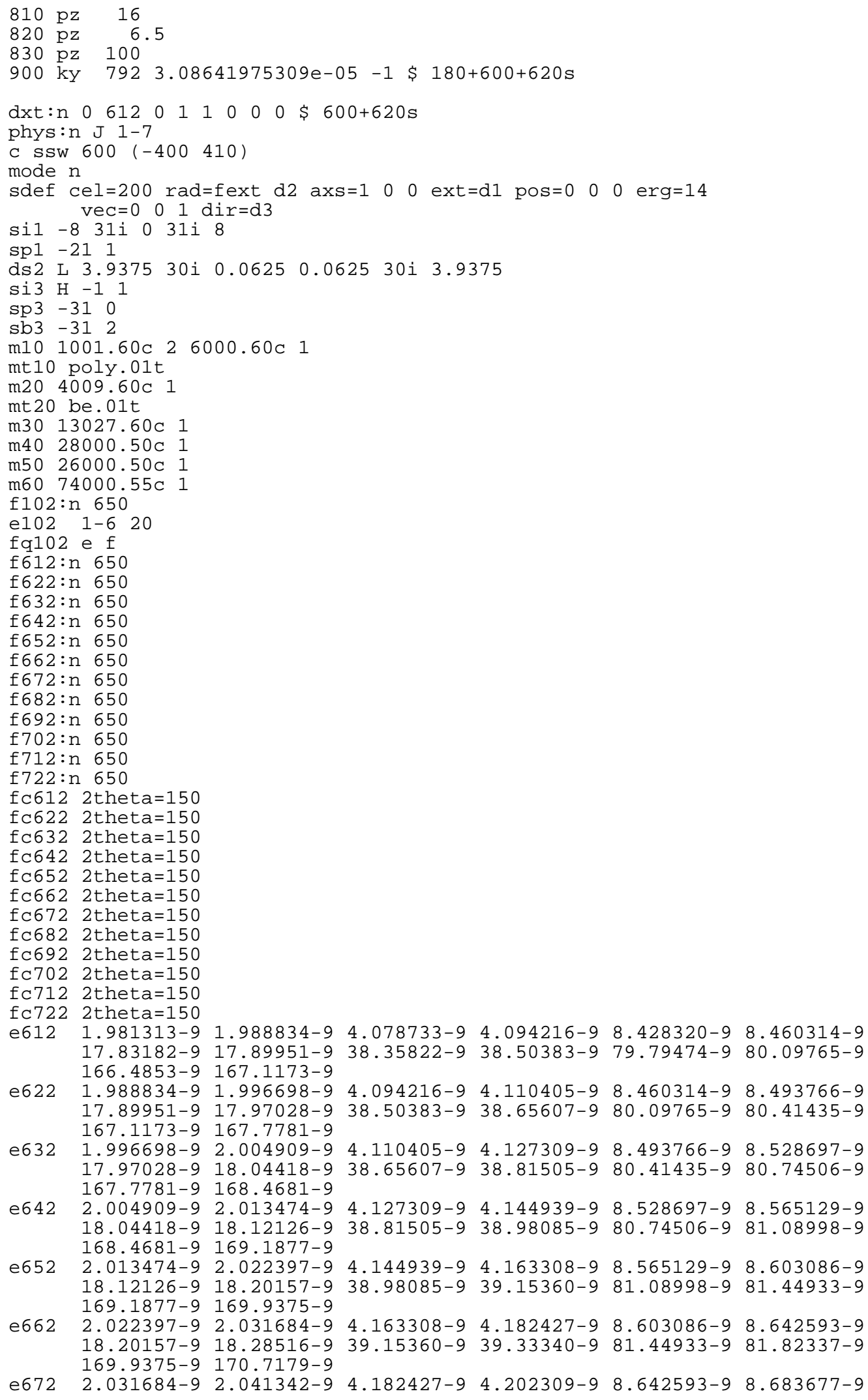

e662 2.022397-9 $2.031684-9 \quad 4.163308-9 \quad 4.182427-9 \quad 8.603086-9 \quad 8.642593-9$

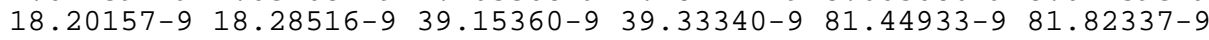
$169.9375-9 \quad 170.7179-9$

e672 2.031684-9 2.041342-9 4 .182427-9 4.202309-9 8.642593-9 8.683677-9 


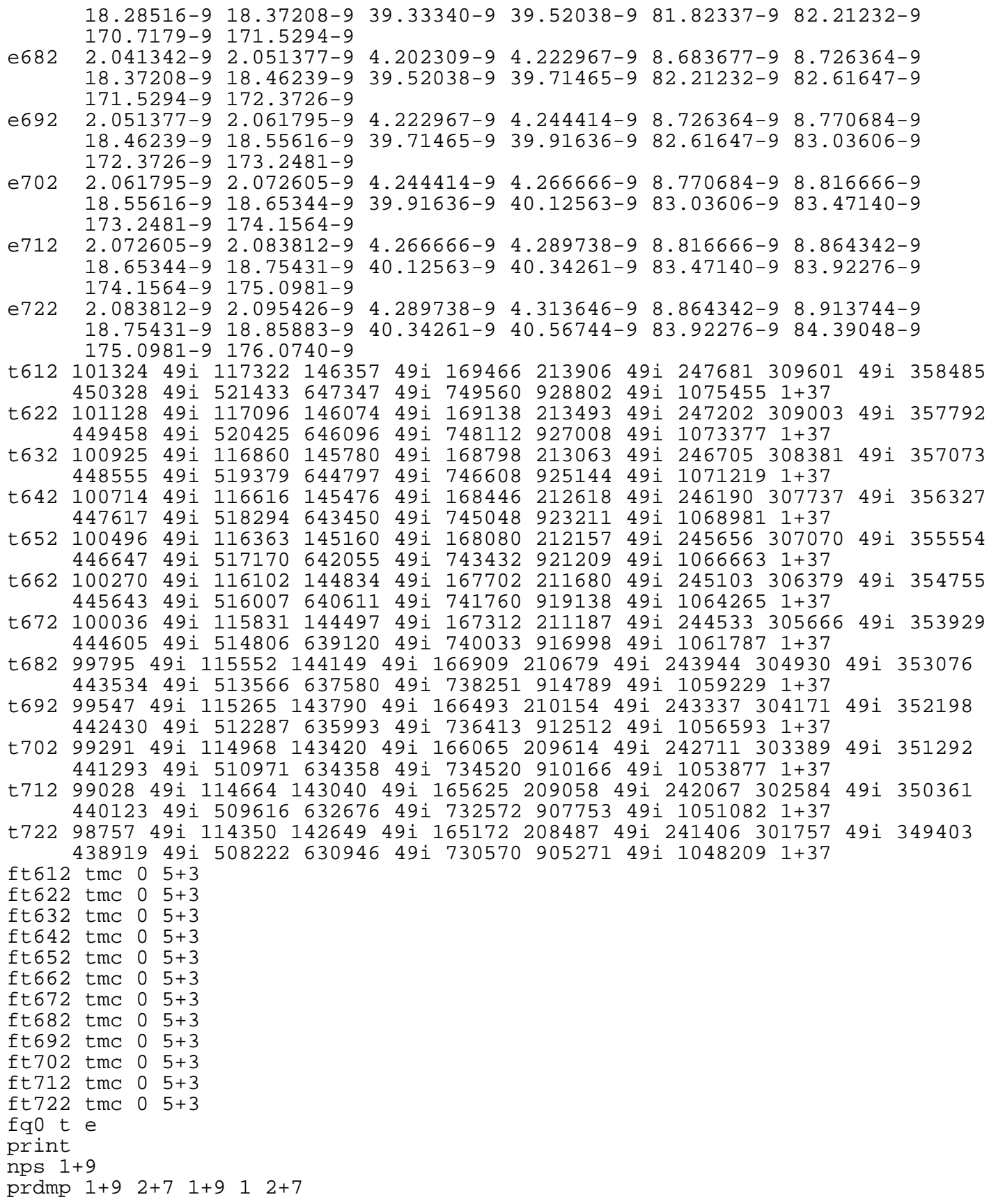

\section{A.4 Cold neutron brightness (with SSW)}

$1 \mathrm{E} 14 \mathrm{D}-\mathrm{T} \mathrm{n} / \mathrm{s}$

$10010-0.071121-100507-300$ imp:n=1 FCL: $n=1$ tmp=1.72344E-09

$10210-0.071121-100505-507$ imp:n=1 FCL: $n=1$ tmp=1.72344E-09

$10520-1.848-100501-505$ imp:n=2

$106 \quad 0-100200-501$ imp: $n=1$

$1100-130-200210$ imp: $n=1$ 


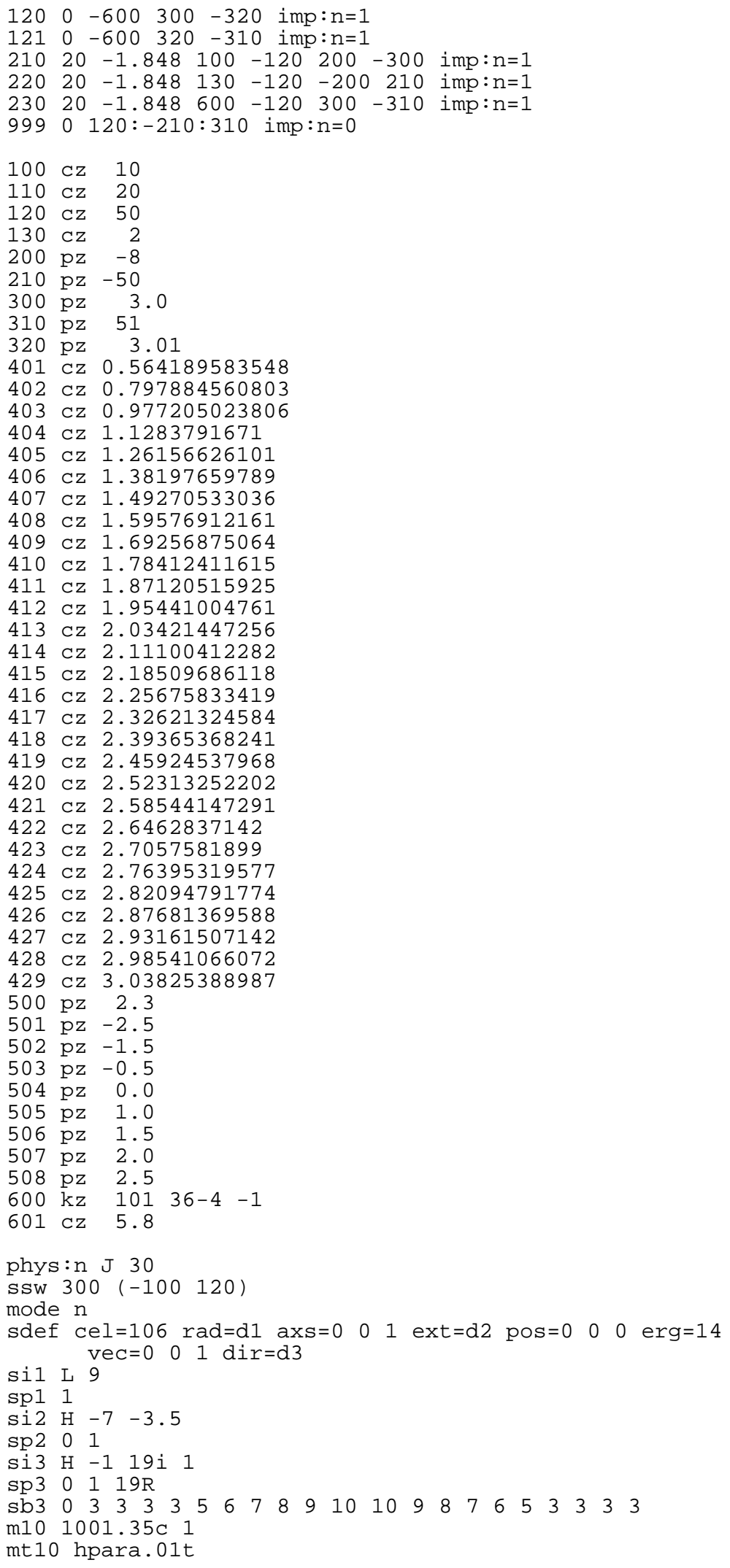


$\mathrm{m} 20 \quad 4009.60 \mathrm{C} 1$

mt20 be. $01 t$

m30 $64000.35 \mathrm{c} 1$

print

nps $1+7$

prdmp $1+7 \quad 5+5 \quad 1+7 \quad 1 \quad 50000$

\section{A.5 Cold neutron brightness (with SSR)}

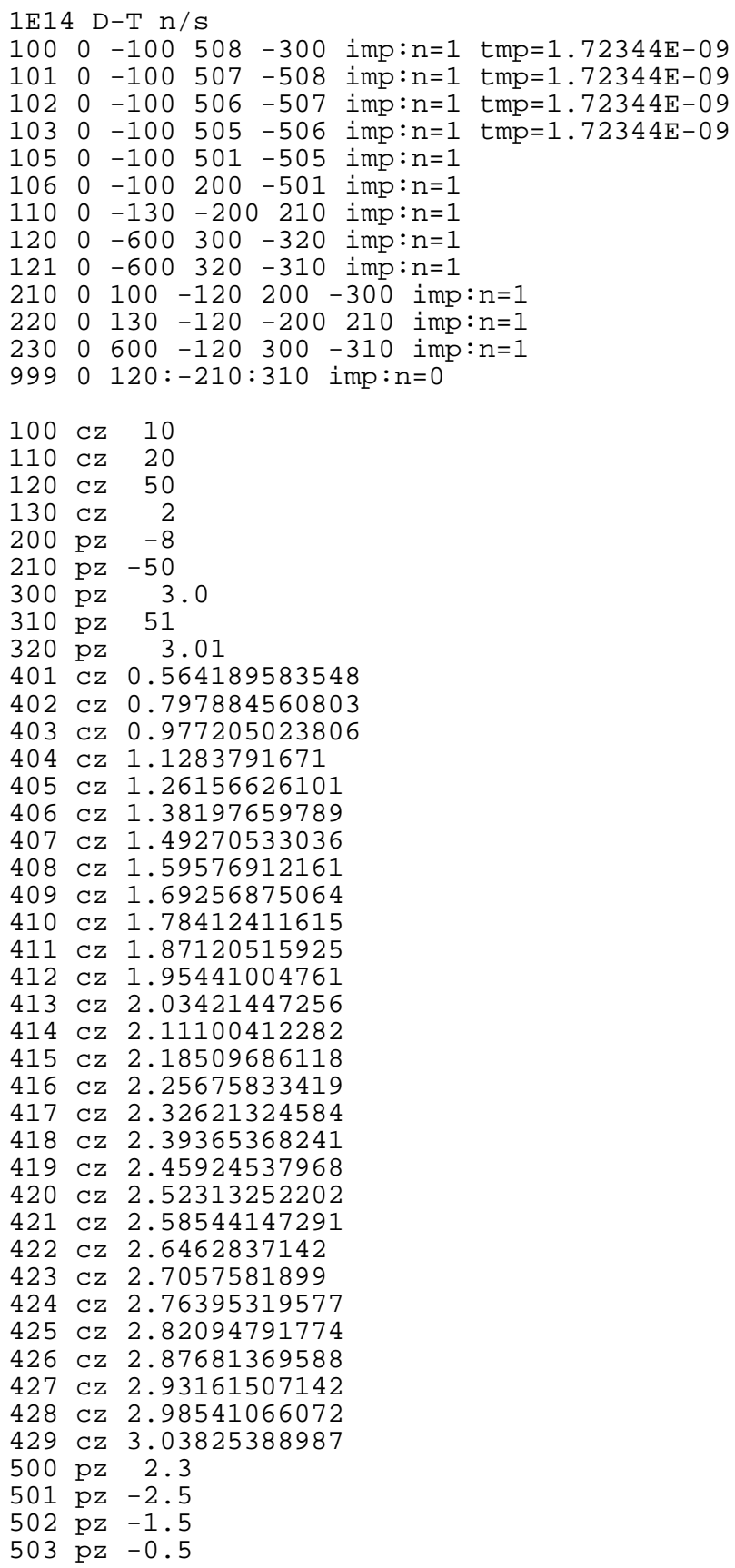




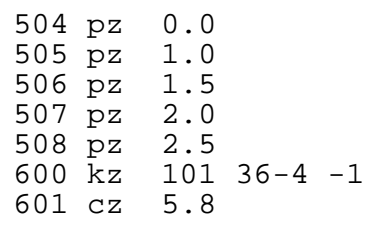

ssr

phys:n J 30

mode $n$

m10 $1001.35 \mathrm{C} 1$

mt10 hpara.01t

$\mathrm{m} 204009.60 \mathrm{c} 1$

mt20 be.01t

m30 $64000.35 \mathrm{C}^{1}$

f102:n 320

sd102 675.385871854

e102 $1-9$ 10-9 $100-9 \quad 500-9 \quad 1-6 \quad 30$

f $302: \mathrm{n} 320$

sd302 675.385871854

e302 1.05925372518-10 $1.1220184543-10 \quad 1.18850222744-10 \quad 1.25892541179-10$

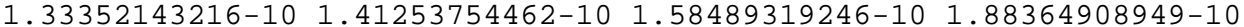
$2.23872113857-10 \quad 2.81838293126-10 \quad 3.75837404288-10 \quad 5.01187233627-10$ $6.68343917569-10 \quad 8.91250938134-10 \quad 1-9$ 1.05925372518-9 $1.1220184543-9$ $1.18850222744-91.25892541179-9 \quad 1.33352143216-9$ $1.41253754462-9 \quad 1.58489319246-9 \quad 1.77827941004-9 \quad 1.99526231497-9$ $\begin{array}{llllll}2.11348903984-9 & 2.23872113857-9 & 2.37137370566-9 & 2.51188643151-9\end{array}$ $2.6607250598-9 \quad 2.81838293126-9 \quad 2.98538261892-9 \quad 3.16227766017-9$ $3.34965439158-9 \quad 3.54813389234-9 \quad 3.75837404288-9 \quad 3.98107170553-9$ $4.21696503429-9 \quad 4.46683592151-9 \quad 4.73151258961-9 \quad 5.01187233627-9$ $5.30884444231-9 \quad 5.6234132519-9 \quad 5.95662143529-9 \quad 6.3095734448-9$ $6.68343917569-97.07945784384-9 \quad 7.49894209332-9 \quad 7.94328234724-9$ $8.41395141645-98.91250938134-9 \quad 9.44060876286-9 \quad 1-8$ $1.05925372518-8 \quad 1.1220184543-8 \quad 1.18850222744-8 \quad 1.25892541179-8$ $1.33352143216-8 \quad 1.41253754462-8 \quad 1.58489319246-8 \quad 1.88364908949-8$ $2.23872113857-8 \quad 2.81838293126-8 \quad 3.75837404288-8 \quad 5.01187233627-8$ $6.68343917569-8 \quad 8.91250938134-8 \quad 1-7 \quad 1.05925372518-7 \quad 1.1220184543-7$ $1.18850222744-7 \quad 1.25892541179-7 \quad 1.33352143216-7 \quad 1.41253754462-7$ $\begin{array}{lllll}1.58489319246-7 & 1.88364908949-7 & 2.23872113857-7 & 2.81838293126-7\end{array}$ $3.75837404288-7 \quad 5.01187233627-7 \quad 6.68343917569-7 \quad 8.91250938134-7$ $1-6 \quad 1.58489319246-6 \quad 2.51188643151-6 \quad 3.98107170553-6 \quad 6.3095734448-6$ $1-51.58489319246-5 \quad 2.51188643151-5 \quad 3.98107170553-5 \quad 6.3095734448-5$ $\begin{array}{llllll}1-4 & 1.58489319246-4 & 2.51188643151-4 & 3.98107170553-4 & 6.3095734448-4\end{array}$ $1-3 \quad 1.58489319246-3 \quad 2.51188643151-3 \quad 3.98107170553-3 \quad 6.3095734448-3$ $1-2$ 1.58489319246-2 2.51188643151-2 $3.98107170553-2 \quad 6.3095734448-2$ 1-1 $1.58489319246-1 \quad 2.51188643151-1 \quad 3.98107170553-1 \quad 6.3095734448-1$ $\begin{array}{llllll}1 & 1.58489319246 & 2.51188643151 & 3.98107170553 & 6.3095734448\end{array}$

fq302 e c $\begin{array}{llll}1+1 & 1.58489319246+1 & 25.1188643151 & 39.8107170553\end{array}$

f $402:$ : 320

sd402 675.385871854

e402 1.0000-9 $1.0140-91.0283-9 \quad 1.0429-9 \quad 1.0578-9 \quad 1.0730-91.0886-9 \quad 1.1045-9$

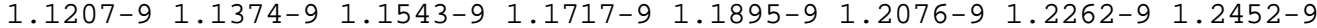
1.2647-9 $1.2846-9$ 1.3050-9 $1.3258-9$ 1.3472-9 $1.3691-9$ 1.3915-9 $1.4145-9$

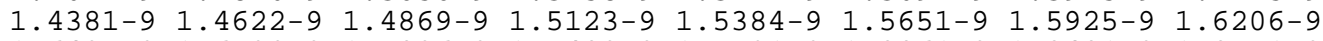

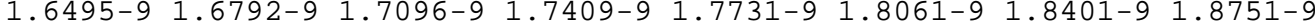
$\begin{array}{llllllll}1.9110-9 & 1.9480-9 & 1.9861-9 & 2.0253-9 & 2.0656-9 & 2.1072-9 & 2.1500-9 & 2.1941-9\end{array}$

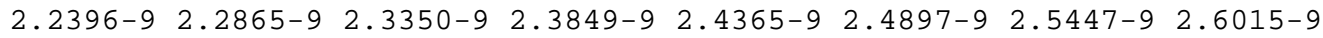
$\begin{array}{llllllll}2.6603-9 & 2.7210-9 & 2.7838-9 & 2.8489-9 & 2.9162-9 & 2.9859-9 & 3.0582-9 & 3.1330-9\end{array}$

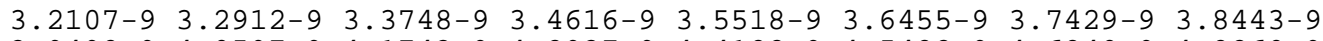

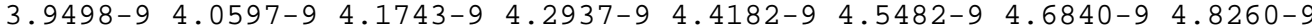

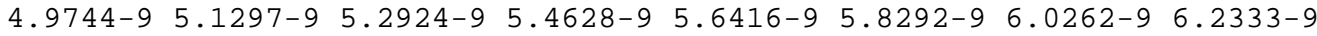

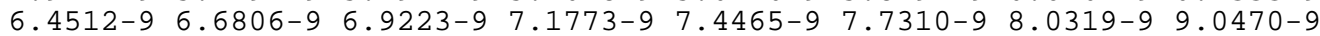

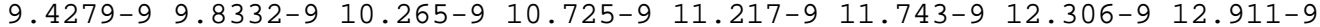

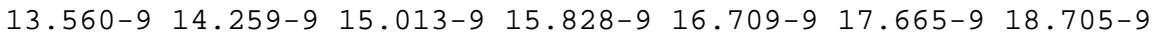

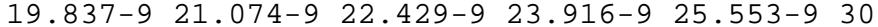

t402 $03991 \quad 1+5 \quad 1+37$

fq402 $t c$

print

prdmp $1+92+6 \quad 1+9 \quad 15000$ 


\section{A.6 D-T neutron generator for positron production}

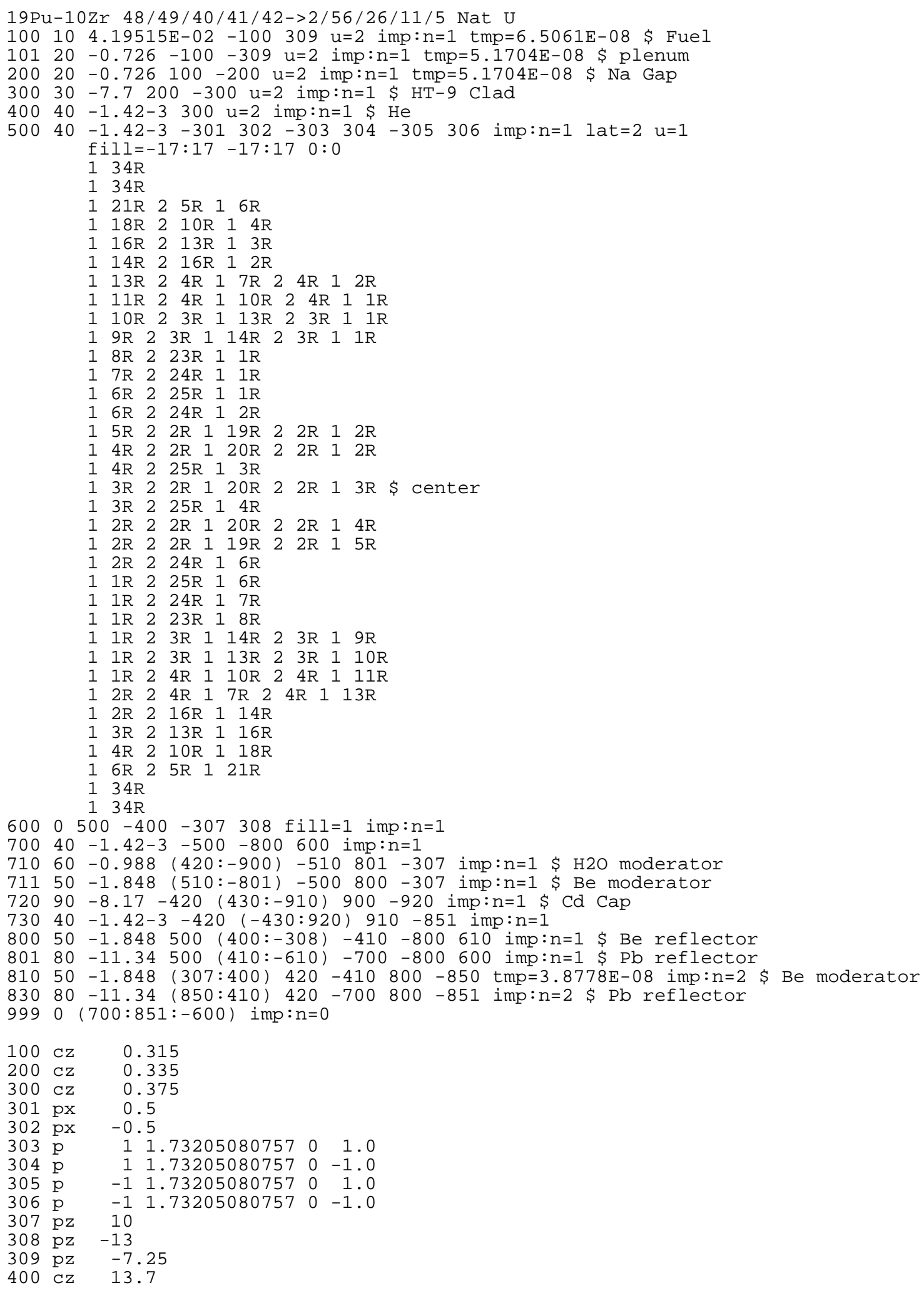




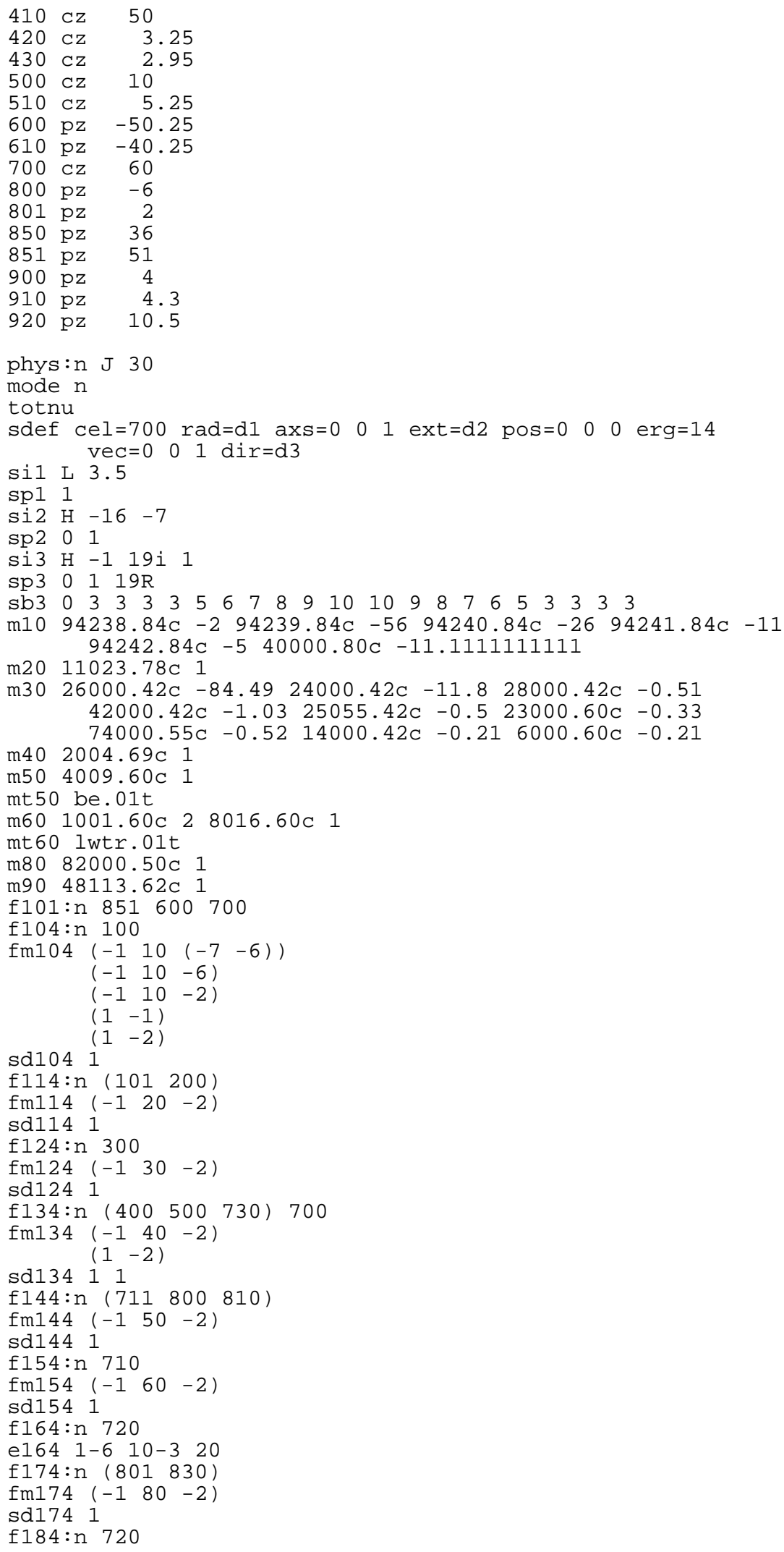




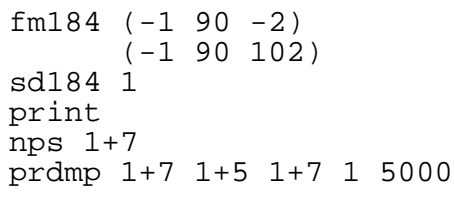

\section{A.7 D-D neutron generator for BNCT}

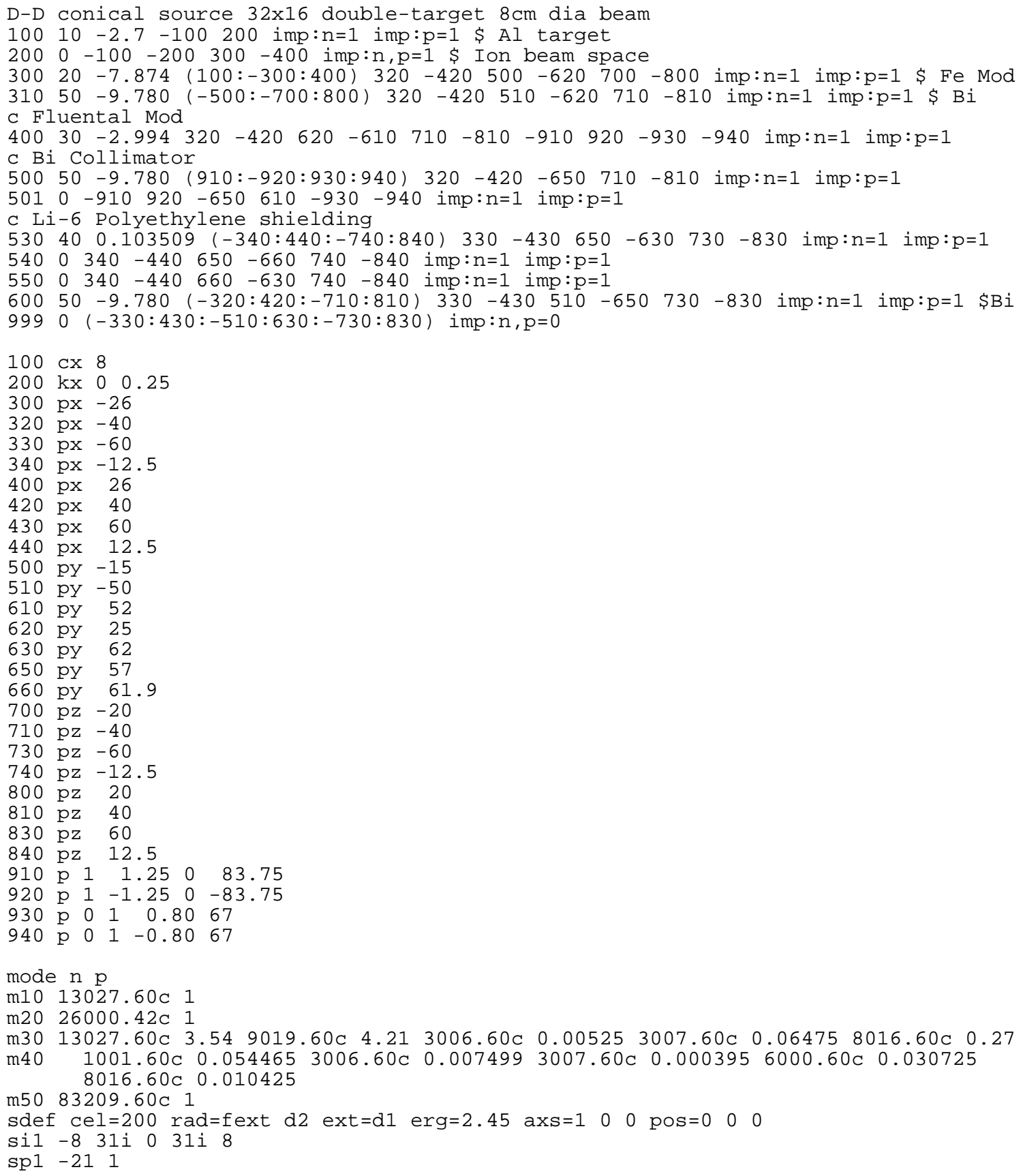




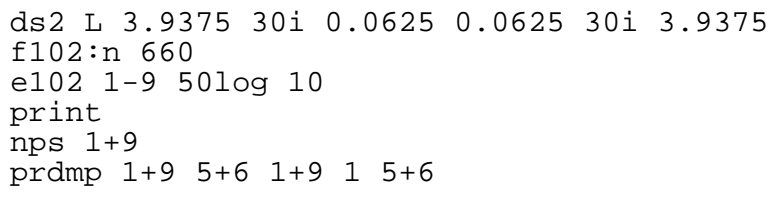

\section{A.8 Fission multiplier for BNCT}

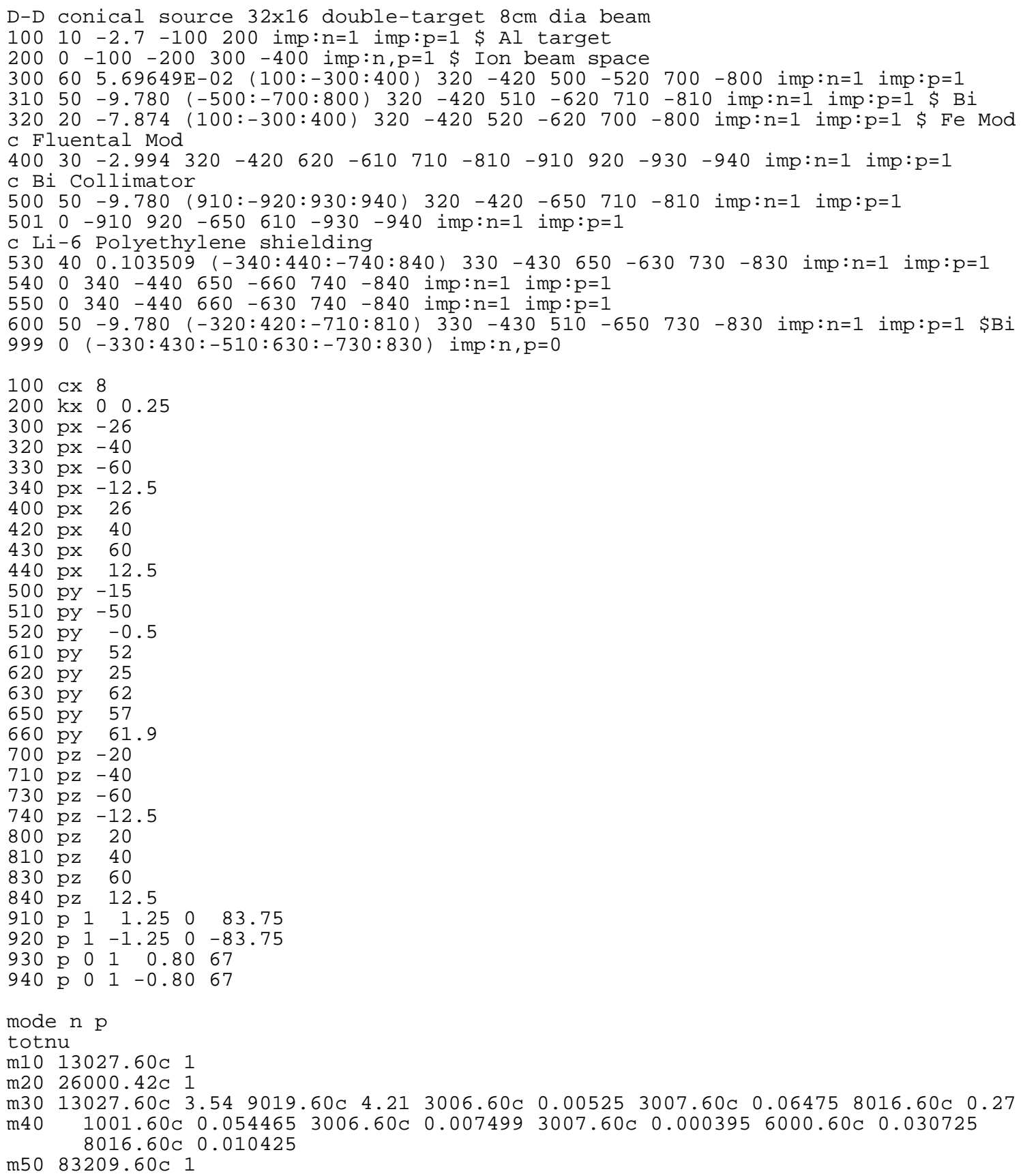




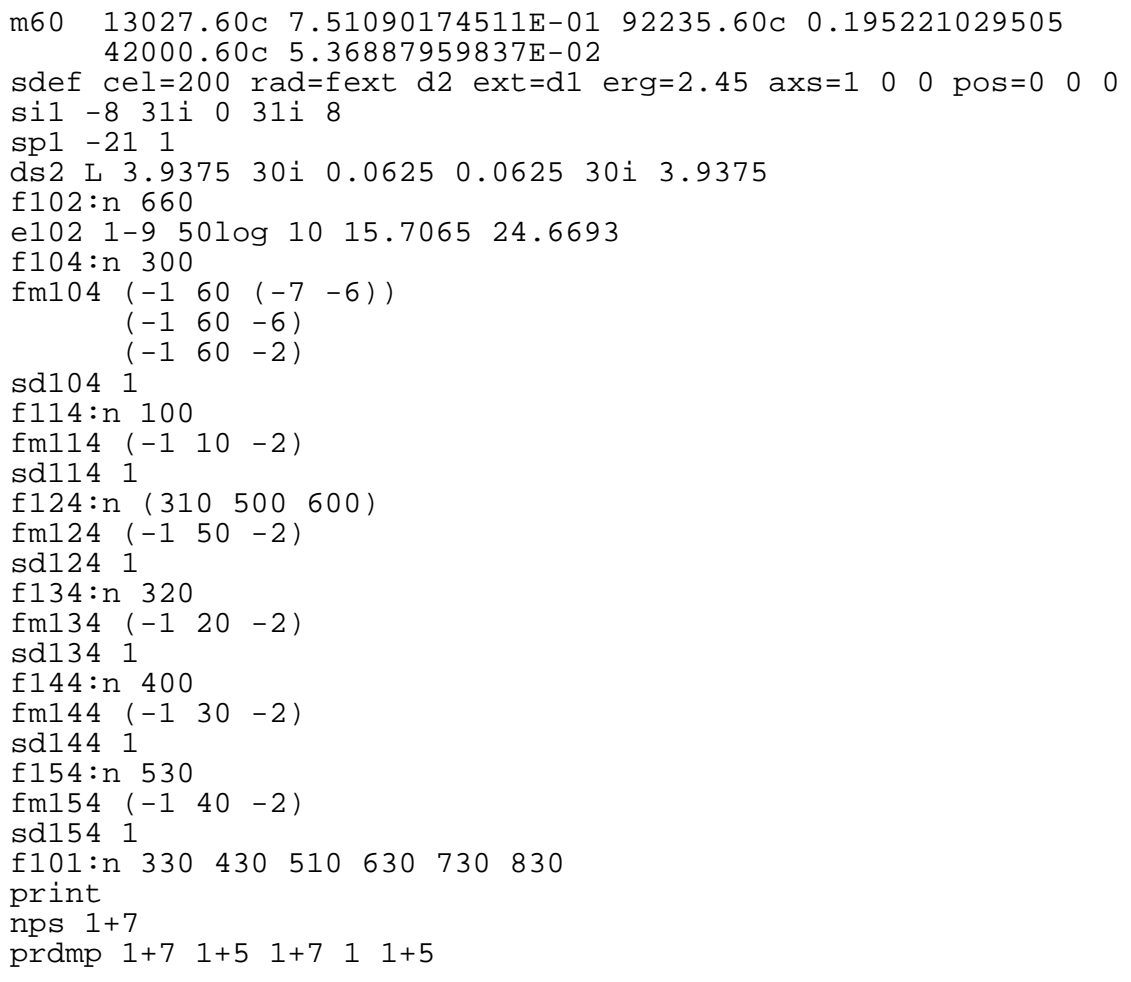

